TRANSACTIONS OF THE

AMERICAN MATHEMATICAL SOCIETY

Volume 349, Number 9, September 1997, Pages 3469-3516

S 0002-9947(97)01793-5

\title{
ON PERFECT ISOMETRIES AND ISOTYPIES IN ALTERNATING GROUPS
}

\author{
PAUL FONG AND MORTON E. HARRIS
}

\begin{abstract}
Perfect isometries and isotypies are constructed for alternating groups between blocks with abelian defect groups and the Brauer correspondents of these blocks. These perfect isometries and isotypies satisfy additional compatibility conditions which imply that an extended Broué conjecture holds for the principal block of an almost simple group with an abelian Sylow $p$ subgroup and a generalized Fitting subgroup isomorphic to an alternating group.
\end{abstract}

Let $G$ be a finite group and let $\mathcal{O}$ be a complete discrete valuation ring with field of quotients $K$ of characteristic 0 and residue class field $k$ of characteristic $p>0$. We suppose that $K$ contains a primitive $|G|$-th root of unity. In [4, (6.1)] Michel Broué posed the following

Isotypy Conjecture. Let e be a block of $\mathcal{O} G$ with abelian defect group $D$ and let $f$ be the Brauer correspondent of e in $\mathcal{O} N_{G}(D)$. Then $e$ and $f$ are isotypic blocks.

If $G$ has an abelian Sylow $p$-subgroup, then the conjecture can be posed for the principal block of $\mathcal{O} G$. In this case the authors have shown that the conjecture holds provided an extended conjecture holds for the principal block of almost simple groups with an abelian Sylow $p$-subgroup (see $[10,(5 \mathrm{E})]$ ). In this paper the isotypy conjecture for an arbitrary block with abelian defect group is proved for alternating groups. In addition, the extended conjecture is proved for the principal $p$-block of almost simple groups with abelian Sylow $p$-subgroups and generalized Fitting subgroup isomorphic to an alternating group.

We recall the basic definitions. Let ${ }^{-}: \mathcal{O} \longrightarrow k$ be the canonical quotient mapping and let ${ }^{-}: \mathcal{O} G \longrightarrow k G$ be the induced $\mathcal{O}$-algebra homomorphism of the group algebras. In particular, ${ }^{-}: e \longrightarrow \bar{e}$ induces a bijection between central idempotents of $\mathcal{O} G$ and $k G$. If $e$ is a block idempotent of $\mathcal{O} G$, let ${ }_{K G e}$ Mod be the category of left $K G e$-modules of finite type and let $\mathcal{R}_{K}(G, e)$ be the Grothendieck group of ${ }_{K G e}$ Mod. Let $G^{\vee}$ be the set of irreducible characters of $G$ over $K$. We identify $\mathcal{R}_{K}(G, e)$ with the free abelian group on

$$
(G, e)^{\vee}=\left\{\chi \in G^{\vee}: \chi(g e)=\chi(g) \text { for all } g \in G\right\} .
$$

Let $\mathrm{CF}(G, K)$ be the $K$-space of $K$-valued class functions on $G$, and let $\operatorname{CF}(G, e, K)$ be the $K$-subspace of class functions $\alpha$ in $\mathrm{CF}(G, K)$ such that $\alpha(g e)=\alpha(g)$. The

Received by the editors March 26, 1996

1991 Mathematics Subject Classification. Primary 20C15, 20C20; Secondary 20C30.

The first author was supported in part by NSF grant DMS 9100310.

The second author was supported in part by NSA grant MDA 904 92-H-3027.

(C)1997 American Mathematical Society 
$K$-subspaces of $\mathrm{CF}(G, K)$ and $\mathrm{CF}(G, e, K)$ of class functions vanishing on the $p$ singular conjugacy classes of $G$ will be denoted by $\mathrm{CF}_{p^{\prime}}(G, K)$ and $\mathrm{CF}_{p^{\prime}}(G, e, K)$.

Similar notation will be used for a second finite group $H$ and a block idempotent $f$ of $\mathcal{O} H$. We denote $\mathcal{R}_{K}(G \times H, e \otimes f)$ also as $\mathcal{R}_{K}((G, e),(H, f))$. Each $\mu$ in $\mathcal{R}_{K}((G, e),(H, f))$ determines adjoint linear maps

$$
\mathrm{R}_{\mu}: \mathrm{CF}(G, e, K) \longrightarrow \mathrm{CF}(H, f, K), \quad \mathrm{I}_{\mu}: \mathrm{CF}(H, f, K) \longrightarrow \mathrm{CF}(G, e, K),
$$

where

$$
\mathrm{R}_{\mu}(\alpha)(h)=\frac{1}{|G|} \sum_{g \in G} \mu\left(g, h^{-1}\right) \alpha\left(g^{-1}\right), \quad \mathrm{I}_{\mu}(\beta)(g)=\frac{1}{|H|} \sum_{h \in H} \mu\left(g, h^{-1}\right) \beta(h)
$$

for $\alpha \in \mathrm{CF}(G, e, K)$ and $\beta \in \mathrm{CF}(H, f, K)$. Moreover, $\mathrm{R}_{\mu}: \mathcal{R}_{K}(G, e) \longrightarrow \mathcal{R}_{K}(H, f)$ and $\mathrm{I}_{\mu}: \mathcal{R}_{K}(H, f) \longrightarrow \mathcal{R}_{K}(G, e)$.

Definition. A virtual character $\mu$ of $G \times H$ is perfect if the following separation and integrality conditions hold for all $g \in G, h \in H$ :

1. $\mu(g, h)=0$ if $\{g, h\}$ consists of a $p$-regular element and a $p$-singular element.

2. $\mu(g, h)$ is divisible in $\mathcal{O}$ by $\left|C_{G}(g)\right|_{p}$ and by $\left|C_{H}(h)\right|_{p}$.

If $\mu$ is perfect, then $\mathrm{R}_{\mu}$ and $\mathrm{I}_{\mu}$ induce $K$-linear mappings

$$
\begin{aligned}
\mathrm{R}_{\mu, p^{\prime}} & : \mathrm{CF}_{p^{\prime}}(G, e, K) \longrightarrow \mathrm{CF}_{p^{\prime}}(H, f, K), \\
\mathrm{I}_{\mu, p^{\prime}}: & \mathrm{CF}_{p^{\prime}}(H, f, K) \longrightarrow \mathrm{CF}_{p^{\prime}}(G, e, K)
\end{aligned}
$$

by restriction. We say $\mu$ is an isometry if $\mathrm{R}_{\mu}$ is an isometry of $\mathcal{R}_{K}(G, e)$ onto $\mathcal{R}_{K}(H, f)$. An isometry $\mu$ is thus a pair $\left(\mathrm{J}_{\mu}, \epsilon_{\mu}\right)$ consisting of a bijection and a sign function

$$
\mathrm{J}_{\mu}:(G, e)^{\vee} \longrightarrow(H, f)^{\vee}, \quad \epsilon_{\mu}:(G, e)^{\vee} \longrightarrow\{ \pm 1\}
$$

such that $\mathrm{R}_{\mu}(\chi)=\epsilon_{\mu}(\chi) \mathrm{J}_{\mu}(\chi)$ for all $\chi \in(G, e)^{\vee}$. In particular,

$$
\mu(g, h)=\sum_{\chi \in(G, e)^{\vee}} \epsilon_{\mu}(\chi)\left(\chi(g) \times \mathrm{J}_{\mu}(\chi)\left(h^{-1}\right)\right) .
$$

It will be convenient to set $G_{P}=C_{G}(P)$ and $G_{x}=C_{G}(x)$ when $P$ is a $p$-subgroup of $G$ and $x$ is a $p$-element of $G$. The Brauer morphism $\mathrm{Br}_{P}^{G}: \mathcal{O} G \longrightarrow k G_{P}$ is the $\mathcal{O}$-linear mapping defined by

$$
\operatorname{Br}_{P}^{G}(g)= \begin{cases}g & \text { if } g \in G_{P}, \\ 0 & \text { if } g \notin G_{P} .\end{cases}
$$

$\operatorname{Br}_{P}^{G}$ preserves the $N_{G}(P)$-conjugation action. Let $e$ and $\bar{e}$ be corresponding blocks of $\mathcal{O} G$ and $k G$. An $\bar{e}$-subpair is a pair $(P, \bar{u})$, where $P$ is a $p$-subgroup of $G, \bar{u}$ is a block of $k G_{P}$, and $(1, \bar{e}) \leq(P, \bar{u})$ is containment in the sense of [1, Definition 3.3]. An $e$-subpair is a pair $(P, u)$, where $P$ is a $p$-subgroup of $G, u$ is a block of $\mathcal{O} G_{P}$, and $(P, \bar{u})$ is an $\bar{e}$-subpair. Containment of such pairs is defined in the obvious way: $(Q, v) \leq(P, u)$ if $(Q, \bar{v}) \leq(P, \bar{u})$. Let $\mathcal{S} p(G, e)$ denote the set of $e$-subpairs of $G$. An $\bar{e}$-element is a pair $(x, \bar{u})$, where $x$ is a $p$-element of $G, \bar{u}$ is a block of $k G_{x}$, and $(\langle x\rangle, \bar{u})$ is an $\bar{e}$-subpair. An $e$-element is a pair $(x, u)$, where $x$ is a $p$-element of $G$, $u$ is a block of $\mathcal{O} G_{x}$, and $(x, \bar{u})$ is an $\bar{e}$-element. The decomposition mapping

$$
d_{G}^{(x, u)}: \mathrm{CF}(G, K) \longrightarrow \mathrm{CF}_{p^{\prime}}\left(G_{x}, u, K\right)
$$


is defined by $d_{G}^{(x, u)}(\alpha)(y)=\alpha(x y u)$ for $\alpha \in \mathrm{CF}(G, K), y \in G_{x, p^{\prime}}$. If $(x, u)$ is an e-element, then $\alpha(x y u)=\alpha(x y e u)$ by Brauer's Second Main Theorem (see [2, $\S \mathrm{A} 2$, Théorème A2.1]).

The objects and morphisms of the Brauer category $\mathbf{B r}_{e}(G)$ are the $e$-subpairs $(P, u)$ of $G$ and the mappings $(P, u) \longrightarrow(Q, v)$ in $\operatorname{Hom}(P, Q)$ induced by conjugation by elements $g$ of $G$ such that $(P, u)^{g} \leq(Q, v)$. Let $\left(D, e_{D}\right)$ be a maximal $e$-subpair of $G$. Each subgroup $P$ of $D$ determines a unique block $e_{P}$ of $\mathcal{O} G_{P}$ such that $\left(P, e_{P}\right) \leq\left(D, e_{D}\right)$. If $P=\langle z\rangle$, we also write $e_{z}$ for $e_{P}$. Let $\mathbf{B r}_{e, D}(G)$ be the full subcategory of $\mathbf{B r}_{e}(G)$ whose objects are such subpairs $\left(P, e_{P}\right)$. Since any two maximal $e$-subpairs are conjugate under $G, \mathbf{B r}_{e, D}(G)$ is independent of the choice of $\left(D, e_{D}\right)$ up to a categorical isomorphism. Moreover, the inclusion functor of $\mathbf{B r}_{e, D}(G)$ into $\mathbf{B r}_{e}(G)$ is a categorical equivalence, since each $e$-subpair of $G$ is conjugate in $G$ to an $e$-subpair $\left(P, e_{P}\right)$ contained in $\left(D, e_{D}\right)$. Similar notation will be used for a block idempotent $f$ of $\mathcal{O} H$ with defect group $D$.

Definition. ([4, Definition 4.3]) Let $e$ and $f$ be block idempotents of $\mathcal{O} G$ and $\mathcal{O} H$ with a common defect group $D$. Let $\left(D, e_{D}\right)$ and $\left(D, f_{D}\right)$ be a maximal $e$-subpair and a maximal $f$-subpair of $G$ and $H$. Then $e$ and $f$ are isotypic if the following conditions hold:

(I1) The inclusion of $D$ in $G$ and $H$ induces an equivalence of the categories $\mathbf{B r}_{e, D}(G)$ and $\mathbf{B r}_{f, D}(H)$.

(I2) There exists a perfect isometry $\mu_{P}$ in $\mathcal{R}_{K}\left(\left(G_{P}, e_{P}\right),\left(H_{P}, f_{P}\right)\right)$ for each cyclic subgroup $P$ of $D$ such that the diagram

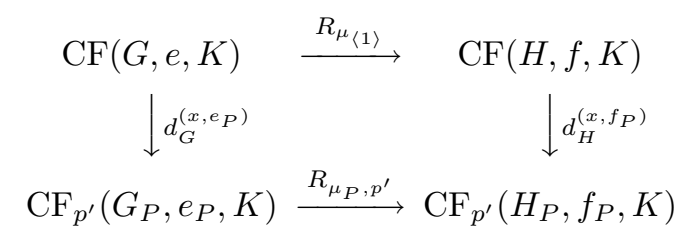

is commutative for every generator $x$ of $P$.

We note that the horizontal arrows in the diagram depend only on $P$, whereas the vertical arrows depend on the generator $x$ of $P$. The set of perfect isometries $\left\{\mu_{P}\right\}$ is called the local system of the isotypy.

Remark. The actual statement of the Isotypy Conjecture in $[4,(6.1)]$ asserts the following: Let $e$ be a block of $\mathcal{O} G$ with abelian defect group $D$, let $\left(D, e_{D}\right)$ be a maximal $e$-subpair of $G$, and let $T=N_{G}\left(D, e_{D}\right)$. Then $e$ and $e_{D}$ are isotypic blocks of $\mathcal{O} G$ and $\mathcal{O} T$. This statement is equivalent to the one given in the introduction of this paper since $f$ and $e_{D}$ are isotypic blocks of $\mathcal{O} H$ and $\mathcal{O} T$ by the following general fact: Suppose $C$ is a normal subgroup of a finite group $H, b$ is a block of $\mathcal{O} C$, and $T=N_{H}(b)$. Let $u$ and $v$ be blocks of $\mathcal{O} H$ and $\mathcal{O} T$ covering $b$ such that $u=\operatorname{Tr}_{T}^{H}(v)$. Then $u$ and $v$ are isotypic blocks of $\mathcal{O} H$ and $\mathcal{O} T$. Indeed, $u$ and $v$ have a common defect group $D$. If $(P, \phi)$ is a $v$-subpair of $T$, then $\left(P, \operatorname{Tr}_{T_{P}}^{H_{P}}(\phi)\right)$ is a $u$-subpair of $H$. The mapping $\mathcal{S} p(T, v) \longrightarrow \mathcal{S} p(H, u)$ defined by $(P, \phi) \mapsto$ $\left(P, \operatorname{Tr}_{T_{P}}^{H_{P}}(\phi)\right)$ implies condition (I1) by [13, Theorem 1] and [4, 4.2, Remark 2]. Let $\left(D, u_{D}\right)$ and $\left(D, v_{D}\right)$ be a maximal $u$-subpair of $H$ and a maximal $v$-subpair of $T$ such that $u_{D}=\operatorname{Tr}_{T_{D}}^{H_{D}}\left(v_{D}\right)$. For each cyclic subgroup $P$ of $D$, the categorical 
equivalences

$$
\begin{aligned}
& \mathcal{O} T_{P} v_{P} \text { Mod } \stackrel{\operatorname{Ind}_{T_{P}}^{H_{P}}}{\longrightarrow} \mathcal{O} H_{P} u_{P} \text { Mod, } \\
& \mathcal{O} H_{P} u_{P} \operatorname{Mod} \stackrel{v_{P} \operatorname{Res}_{T_{P}}^{H_{P}}}{\longrightarrow} \mathcal{O} T_{P} v_{P} \text { Mod }
\end{aligned}
$$

induce perfect isometries $\mu_{P}$ in $\mathcal{R}_{K}\left(\left(H_{P}, u_{P}\right),\left(T_{P}, v_{P}\right)\right)$ satisfying $(\mathbf{I 2})$.

The isotypies for the alternating group $A_{n}$ will be constructed from isotypies for the symmetric group $S_{n}$. In $\S 1$ we consider a configuration $\left(G, G^{+}, H, H^{+}\right)$of finite groups, where $G^{+}$is normal in $G, H^{+}$is normal in $H, G / G^{+} \simeq H / H^{+}$; a configuration $\left(e, e^{+}, f, f^{+}\right)$of blocks of $\mathcal{O} G, \mathcal{O} G^{+}, \mathcal{O} H, \mathcal{O} H^{+}$, where $e$ covers $e^{+}$ and $f$ covers $f^{+}$; and an isotypy between $e$ and $f$ with local system $\left\{\mu_{P}\right\}$. An isotypy between $e^{+}$and $f^{+}$can be constructed under suitable conditions on $\left\{\mu_{P}\right\}$. In the given context the relevant condition is that of +equivariance considered in $\S 2$. This construction will then be applied to configurations where $G=S_{n}$, $G^{+}=A_{n}, e^{+}$is a block of $\mathcal{O} G^{+}$with abelian defect group $D^{+}, H=N_{G}\left(D^{+}\right)$, $H^{+}=N_{G^{+}}\left(D^{+}\right)$, and $f^{+}$is the Brauer correspondent of $e^{+}$. If $p$ is odd, then $e$ and $f$ have $D^{+}$as defect group. In this case Rouquier ([14, Théorème 2.13]) has shown that $e$ and $f$ are isotypic blocks. However, the isotypies in [Ro] are not explicit enough to check the condition of +equivariance. We therefore give in $\S 3$ an explicit +-equivariant isotypy between $e$ and $f$. $\S 4$ contains the relevant character theory of $H^{+}$. The main result there is an analog of Frobenius's theorem on irreducible characters of $G^{+}$which do not extend to characters of $G$. $\S 5$ contains the construction of the isotypy between $e^{+}$and $f^{+}$when $p>2$, and $\S 6$ contains the proof that this isotypy satisfies the extended conjecture of $[10,(5 \mathrm{E})]$ when $e^{+}$ and $f^{+}$are the principal blocks. $\S 7$ treats the case $p=2$.

\section{Some CONFIGURATIONS OF ISOTYPIES}

An isotypy between blocks $e$ and $f$ of $\mathcal{O} G$ and $\mathcal{O} H$ with a common defect group $D$ requires a perfect isometry $\mu_{P}$ for each cyclic subgroup $P$ of $D$ such that $(0.1)$ is commutative for every generator $x$ of $P$. In practice somewhat less is required. The following reduction steps will be used in our proofs.

Step 1. Suppose $e$ and $f$ are blocks of principal type, that is, $\operatorname{Br}_{P}^{G}(e)$ and $\operatorname{Br}_{P}^{H}(f)$ are blocks of $\mathcal{O} G_{P}$ and $\mathcal{O} H_{P}$ for each subgroup $P$ of $D$. Then the decomposition mappings $d_{G}^{\left(x, e_{x}\right)}$ and $d_{H}^{\left(x, f_{x}\right)}$ in (0.1) are simply $d_{G}^{x}$ and $d_{H}^{x}$ by Brauer's Second Main Theorem.

Step 2. $\mu_{\langle 1\rangle}$ need only be an isometry, since $\mu_{\langle 1\rangle}$ is perfect provided the other conditions hold [4, Remark following 4.6].

Step 3. If (I1) holds, the commutativity of (0.1) need only be checked for $\left(P, e_{P}\right)$ with $P$ in a set $\mathcal{P}$ of representatives for the $N_{G}\left(D, e_{D}\right)$-conjugacy classes of nontrivial cyclic subgroups of $D$ and for all generators $x$ of such $P$. This follows by transport of structure. For suppose (0.1) commutes for all generators $x$ of such a $P$. Fix $g \in N_{G}\left(D, e_{D}\right)$ and let $Q=P^{g}$, so that $e_{Q}=\left(e_{P}\right)^{g}$. Choose $h \in N_{H}\left(D, f_{D}\right)$ so that $h$ and $g$ induce the same automorphism of $D$. Then $Q=P^{h}$ and $f_{Q}=\left(f_{P}\right)^{h}$. Define the perfect isometry $\mu_{Q}$ in $\mathcal{R}_{K}\left(\left(G_{Q}, e_{Q}\right),\left(H_{Q}, f_{Q}\right)\right)$ by $\mu_{Q}(y, z)=\mu_{P}\left(g y g^{-1}, h z h^{-1}\right)$ for $y \in G_{Q}, z \in H_{Q}$. Then (0.1) with $P$ replaced by $Q$ and $x$ replaced by any generator of $Q$ is commutative. 
For the rest of this section $\sigma$ is a fixed linear character of $G$. Multiplication by $\sigma$ gives an action $\chi \mapsto \sigma \chi$ on $G^{\vee}$; an action $e \mapsto \sigma e$ on central idempotents of $\mathcal{O} G$, where $\sigma e=\sum_{g \in G_{p^{\prime}}} \sigma\left(g^{-1}\right) c_{g} g$ if $e=\sum_{g \in G_{p^{\prime}}} c_{g} g$; and an action $\bar{e} \mapsto \sigma \bar{e}$ on central idempotents of $k G$, where $\sigma \bar{e}=\sum_{g \in G_{p^{\prime}}} \bar{\sigma}\left(g^{-1}\right) \bar{c}_{g} g$ if $\bar{e}=\sum_{g \in G_{p^{\prime}}} \bar{c}_{g} g$. In particular, $\sigma \bar{e}=\overline{\sigma e}$. If $e$ is a block idempotent of $\mathcal{O} G$, then $\chi \in(G, e)^{\vee}$ if and only if $\sigma \chi \in(G, \sigma e)^{\vee}$. Moreover, $e$ and $\sigma e$ have the same defect groups, and $\sigma e=e$ if $\sigma$ has $p$-power order. Let $e$ be a fixed block of $\mathcal{O} G$. If $P$ is a $p$-subgroup of $G$, set $\sigma_{P}=\operatorname{Res}_{G_{P}}^{G}(\sigma)$ for simplicity of notation. In particular, $\operatorname{Br}_{P}^{G}(\sigma e)=$ $\sigma_{P} \operatorname{Br}_{P}^{G}(e)$. If $(P, u)$ is an $e$-subpair of $G$, then $\left(P, \sigma_{P} u\right)$ is a $\sigma e$-subpair of $G$. More generally, let $(P, u)$ and $(Q, v)$ be subpairs of $G$. Then $(P, u) \leq(Q, v)$ if and only if $\left(P, \sigma_{P} u\right) \leq\left(Q, \sigma_{Q} v\right)$. Indeed, we need only show this for normal containment, since containment of subpairs is by definition a chain of normal containments. Let $(P, u) \triangleleft(Q, v)$. Then $P \triangleleft Q, u$ is stable under $Q$, and $\operatorname{Br}_{Q}^{G_{P} Q}(u) v=v$. So $\sigma_{P} u$ is stable under $Q$ and $\operatorname{Br}_{Q}^{G_{P} Q}\left(\operatorname{Res}_{G_{P} Q}^{G}(\sigma) u\right) \cdot \sigma_{Q} v=\sigma_{Q} v$. Thus $\left(P, \sigma_{P} u\right) \triangleleft\left(Q, \sigma_{Q} v\right)$. A similar argument using $\sigma^{-1}$ shows that if $\left(P, \sigma_{P} u\right) \triangleleft\left(Q, \sigma_{Q} v\right)$, then $(P, u) \triangleleft(Q, v)$.

Lemma (1A). $\mathbf{B r}_{e, D}(G)$ and $\mathbf{B} \mathbf{r}_{\sigma e, D}(G)$ are isomorphic categories under the inclusion of a common defect group $D$ of $e$ and $\sigma e$ in $G$.

Proof. We fix a maximal $e$-subpair $\left(D, e_{D}\right)$ of $G$ and the corresponding maximal $\sigma e$-subpair $\left(D, \sigma_{D} e_{D}\right)$ of $G$. If $P \leq D$ and $\left(P, e_{P}\right) \leq\left(D, e_{D}\right)$, then $\left(P, \sigma_{P} e_{P}\right) \leq$ $\left(D, \sigma_{D} e_{D}\right)$ and $\sigma_{P} e_{P}=(\sigma e)_{P}$. To complete the argument, we show that

$$
\operatorname{Hom}\left(\left(P, e_{P}\right),\left(Q, e_{Q}\right)\right)=\operatorname{Hom}\left(\left(P, \sigma_{P} e_{P}\right),\left(Q, \sigma_{Q} e_{Q}\right)\right)
$$

as subsets of $\operatorname{Hom}(P, Q)$. If $\left(P, e_{P}\right)^{g}=\left(P^{g},\left(e_{P}\right)^{g}\right) \leq\left(Q, e_{Q}\right)$ for $g \in G$, then $\left(P, \sigma_{P} e_{P}\right)^{g}=\left(P^{g}, \sigma_{P^{g}} e_{P^{g}}\right) \leq\left(Q, \sigma_{Q} e_{Q}\right)$ and $g \in \operatorname{Hom}\left(\left(P, \sigma_{P} e_{P}\right),\left(Q, \sigma_{Q} e_{Q}\right)\right)$. A similar argument using the linear character $\sigma^{-1}$ gives the reverse inclusion.

Proposition (1B). Suppose $\sigma$ is trivial on a defect group $D$ of e and $\sigma e$. Then $e$ and $\sigma e$ are isotypic blocks with local system $\left\{\mu_{P}\right\}$, where $\mu_{P}=\left(J_{P}, \epsilon_{P}\right)$ for each cyclic subgroup $P$ of $D$ is the isometry in $\mathcal{R}_{K}\left(\left(G_{P}, e_{P}\right),\left(G_{P}, \sigma_{P} e_{P}\right)\right)$ such that $J_{P}(\zeta)=\sigma_{P} \zeta$ and $\epsilon_{P}(\zeta)=1$ for $\zeta \in\left(G_{P}, e_{P}\right)^{\vee}$.

Proof. Condition (I1) for an isotypy holds by (1A). The isometry $\mu_{P}$ is perfect since

$$
\mu_{P}(x, y)=\sum_{\zeta \in\left(G_{P}, e_{P}\right)^{\vee}} \zeta(x) \sigma_{P}\left(y^{-1}\right) \zeta\left(y^{-1}\right)=\sigma_{P}\left(y^{-1}\right) \sum_{\zeta \in\left(G_{P}, e_{P}\right)^{\vee}} \zeta(x) \zeta\left(y^{-1}\right)
$$

and the identity isometry of $\left(G_{P}, e_{P}\right)^{\vee}$ is perfect. The diagram

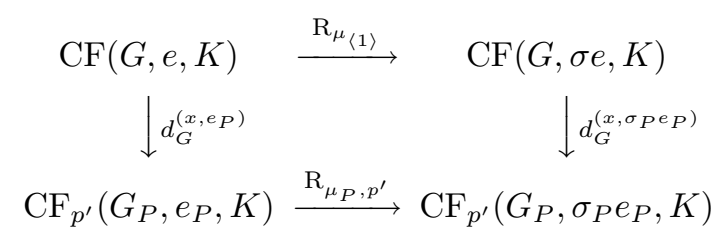


is commutative for each cyclic subgroup $P$ of $D$ and for every generator $x$ of $P$. Indeed, let $\chi \in(G, e)^{\vee}$ and $x^{\prime} \in G_{P, p^{\prime}}$. Then

$$
\begin{aligned}
d_{G}^{\left(x, \sigma_{P} e_{P}\right)}\left(\mathrm{R}_{\mu_{\langle 1\rangle}}(\chi)\right)\left(x^{\prime}\right) & =d_{G}^{\left(x, \sigma_{P} e_{P}\right)}(\sigma \chi)\left(x^{\prime}\right) \\
& =(\sigma \chi)\left(x x^{\prime} \sigma_{P} e_{P}\right), \\
\mathrm{R}_{\mu_{P}, p^{\prime}}\left(d_{G}^{\left(x, e_{P}\right)}(\chi)\right)\left(x^{\prime}\right) & =\left(\sigma_{P} \cdot d_{G}^{\left(x, e_{P}\right)}(\chi)\right)\left(x^{\prime}\right) \\
& =\sigma\left(x^{\prime}\right) \chi\left(x x^{\prime} e_{P}\right) .
\end{aligned}
$$

Now

$$
x x^{\prime} \sigma_{P} e_{P}=\frac{1}{\left|G_{P}\right|} \sum_{g \in G_{P}} \sum_{\zeta \in\left(G_{P}, e_{P}\right)^{\vee}} \zeta(1) \sigma\left(g^{-1}\right) \zeta\left(g^{-1}\right) x x^{\prime} g
$$

Thus

$$
\begin{aligned}
(\sigma \chi)\left(x x^{\prime} \sigma_{P} e_{P}\right) & =\frac{1}{\left|G_{P}\right|} \sum_{g \in G_{P}} \sum_{\zeta \in\left(G_{P}, e_{P}\right)^{\vee}} \zeta(1) \sigma\left(g^{-1}\right) \zeta\left(g^{-1}\right) \sigma\left(x x^{\prime} g\right) \chi\left(x x^{\prime} g\right) \\
& =\frac{1}{\left|G_{P}\right|} \sum_{g \in G_{P}} \sum_{\zeta \in\left(G_{P}, e_{P}\right)^{\vee}} \sigma\left(x x^{\prime}\right) \zeta(1) \zeta\left(g^{-1}\right) \chi\left(x x^{\prime} g\right) \\
& =\frac{1}{\left|G_{P}\right|} \sum_{g \in G_{P}} \sum_{\zeta \in\left(G_{P}, e_{P}\right)^{\vee}} \sigma\left(x^{\prime}\right) \zeta(1) \zeta\left(g^{-1}\right) \chi\left(x x^{\prime} g\right) \quad \text { since } \sigma(x)=1 \\
& =\sigma\left(x^{\prime}\right) \chi\left(x x^{\prime} e_{P}\right)
\end{aligned}
$$

and (1B) holds.

For the rest of this section we suppose that $\langle\sigma\rangle$ acts regularly on the orbit of $e$, i.e., $\sigma^{i} e=e$ if and only if $\sigma^{i}=1$. Set $G^{+}=\operatorname{ker} \sigma$ and $t=\left|G / G^{+}\right|$. Then $G^{+}$ contains the defect group $D$ of $e$, since $t$ is relatively prime to $p$. If $\chi \in(G, e)^{\vee}$, then $\sigma^{i} \chi \in\left(G, \sigma^{i} e\right)^{\vee}$ and the $t$ characters $\sigma^{i} \chi$ are distinct. Thus $\operatorname{Res}_{G^{+}}^{G}(\chi)$ is irreducible and the $\sigma^{i} e$ cover a single block $e^{+}$of $\mathcal{O} G^{+}$. In other words, $e^{+}=\sum_{i=1}^{t} \sigma^{i} e$ and $e^{+}$is $G$-stable. Thus the restriction $\operatorname{Res}_{G^{+}}^{G}$ induces a perfect isometry $\rho=\left(\mathrm{J}_{\rho}, \epsilon_{\rho}\right)$ in $\mathcal{R}_{K}\left((G, e),\left(G^{+}, e^{+}\right)\right)$, where $\mathrm{J}_{\rho}(\chi)=\operatorname{Res}_{G}^{G}(\chi)$ and $\epsilon_{\rho}(\chi)=1$ for $\chi \in(G, e)^{\vee}$.

A subpair $(P, u)$ of $G$ covers a subpair $\left(P, u^{+}\right)$of $G^{+}$if $u$ covers $u^{+}$. Suppose $(P, u)$ is an $e$-subpair. Then $\operatorname{Br}_{P}^{G}(e) u=u$, so that $\operatorname{Br}_{P}^{G}\left(\sigma^{i} e\right) \cdot \sigma_{P}^{i} u=\sigma_{P}^{i} u$. This implies $\left\langle\sigma_{P}\right\rangle$ acts regularly on the orbit of $u$. Moreover, $G_{P}^{+}=G_{P} \cap G^{+}$is the kernel of $\sigma_{P}, G=G^{+} G_{P}$, and $G / G^{+} \simeq G_{P} / G_{P}^{+}$. In particular, $\operatorname{Res}_{G_{P}^{+}}^{G_{P}}$ induces a perfect isometry $\rho_{P}=\left(\mathrm{J}_{\rho_{P}}, \epsilon_{\rho_{P}}\right)$ in $\mathcal{R}_{K}\left(\left(G_{P}, e_{P}\right),\left(G_{P}^{+}, e_{P}^{+}\right)\right)$.

Lemma (1C). Suppose $\langle\sigma\rangle$ acts regularly on the orbit of e. Then the following hold:

(i) Each $e^{+}$-subpair $\left(P, u^{+}\right)$of $G^{+}$is covered by a unique e-subpair $(P, u)$ of G.

(ii) Each e-subpair $(P, u)$ of $G$ covers a unique $e^{+}$-subpair $\left(P, u^{+}\right)$of $G^{+}$.

Proof. We have $\operatorname{Br}_{P}^{G^{+}}\left(e^{+}\right)=\operatorname{Br}_{P}^{G}\left(e^{+}\right)=\sum_{i=1}^{t} \operatorname{Br}_{P}^{G}\left(\sigma^{i} e\right)$ since $e^{+}=\sum_{i=1}^{t} \sigma^{i} e$. If $\left(P, u^{+}\right)$is an $e^{+}$-subpair of $G^{+}$, then $\sum_{i} \operatorname{Br}_{P}^{G}\left(\sigma^{i} e\right) u^{+}=u^{+}$and $\operatorname{Br}_{P}^{G}\left(\sigma^{i} e\right) u^{+} \neq 0$ for some $i$. So there exists a block $u$ of $\mathcal{O} G_{P}$ such that $\sigma_{P}^{i} u$ covers $u^{+}$and $\operatorname{Br}_{P}^{G}\left(\sigma^{i} e\right)$. $\sigma_{P}^{i} u=\sigma_{P}^{i} u$. This implies $\operatorname{Br}_{P}^{G}\left(\sigma^{j} e\right) \cdot \sigma_{P}^{j} u=\sigma_{P}^{j} u$ for all $j$. In particular, $\left\langle\sigma_{P}\right\rangle$ 
acts regularly on the orbit of $u, u^{+}=\sum_{i=1}^{t} \operatorname{Res}_{G_{P}^{+}}^{G_{P}}\left(\sigma_{P}^{i}\right) u$, and $(P, u)$ is the unique $e$-subpair of $G$ covering $\left(P, u^{+}\right)$. Conversely, suppose $(P, u)$ is an $e$-subpair of $G$. Then $\left\langle\sigma_{P}\right\rangle$ acts regularly on the orbit of $u, u$ covers a unique block $u^{+}$of $\mathcal{O} G_{P}^{+}$, and $u^{+}=\sum_{i=1}^{t} \sigma_{P}^{i} u$. Since $\operatorname{Br}_{P}^{G^{+}}\left(e^{+}\right)=\sum_{i=1}^{t} \operatorname{Br}_{P}^{G}\left(\sigma^{i} e\right)$, it follows that $\operatorname{Br}_{P}^{G^{+}}\left(e^{+}\right) u^{+}=$ $u^{+}$. Thus $\left(P, u^{+}\right)$is the unique $e^{+}$-subpair of $G^{+}$covered by $(P, u)$.

Proposition (1D). Suppose $\langle\sigma\rangle$ acts regularly on the orbit of $e$ and $D$ is a defect group of $e$. Then $e$ and $e^{+}$are isotypic blocks with local system $\left\{\rho_{P}: P \leq\right.$ D, $P$ cyclic $\}$.

Proof. By (1C), $u$ covers $u^{+}$if and only if there is a bijection $\gamma: \mathcal{S} p\left(G^{+}, e^{+}\right) \longrightarrow$ $\mathcal{S} p(G, e)$ such that $\gamma\left(\left(P, u^{+}\right)\right)=(P, u)$. To show condition (I1) for an isotypy it suffices to show that $\gamma$ satisfies the following conditions (see [4, 4.2, Remark 2]):

(C1) $\gamma$ is injective and $\gamma\left(\left(P, u^{+}\right)^{h}\right)=\gamma\left(\left(P, u^{+}\right)\right)^{h}$ for all $h \in G^{+}$.

(C2) Every element in $\mathcal{S} p(G, e)$ is $G$-conjugate to $\gamma\left(\left(P, u^{+}\right)\right)$for some element $\left(P, u^{+}\right)$in $\mathcal{S} p\left(G^{+}, e^{+}\right)$.

(C3) If $\gamma\left(\left(Q, v^{+}\right)\right)=\gamma\left(\left(P, u^{+}\right)\right)^{g}$ for $\left(P, u^{+}\right)$and $\left(Q, v^{+}\right)$in $\mathcal{S} p\left(G^{+}, e^{+}\right)$and $g$ in $G$, then there exist $z \in G_{P}$ and $h \in G^{+}$such that $g=z h$.

(C4) $\gamma\left(\left(P, u^{+}\right)\right) \leq \gamma\left(\left(Q, v^{+}\right)\right)$if and only if $\left(P, u^{+}\right) \leq\left(Q, v^{+}\right)$.

Conditions (C1), (C2), (C3) follow easily from (1C) and the factorization $G=$ $G_{P} G^{+}$. Suppose $\gamma\left(\left(P, u^{+}\right)\right)=(P, u), \gamma\left(\left(Q, v^{+}\right)\right)=(Q, v)$, and $\left(P, u^{+}\right) \leq\left(Q, v^{+}\right)$. We claim $(P, u) \leq(Q, v)$. It suffices to show this for normal containment. If $\left(P, u^{+}\right) \triangleleft\left(Q, v^{+}\right)$, then $P \triangleleft Q, u^{+}$is stable under $Q$, and $\operatorname{Br}_{Q}^{G_{P}^{+} Q}\left(u^{+}\right) v^{+}=v^{+}$. Thus $u$ is stable under $Q$ by $(1 \mathrm{C})$. Moreover, $u$ covers $u^{+}$viewed as blocks of $\mathcal{O} G_{P} Q$ and $\mathcal{O} G_{P}^{+} Q$, and $u^{+}=\sum_{i} \operatorname{Res}_{G_{P} Q}^{G}\left(\sigma^{i}\right) u$. Since $\operatorname{Br}_{Q}^{G_{P} Q}\left(\operatorname{Res}_{G_{P} Q}^{G}\left(\sigma^{i}\right) u\right) \cdot \sigma_{Q}^{j} v=0$ for $i \neq j$ and since $v^{+}=\sum_{j} \sigma_{Q}^{j} v$, it follows that

$$
v^{+}=\operatorname{Br}_{Q}^{G_{P}^{+} Q}\left(u^{+}\right) v^{+}=\sum_{i} \operatorname{Br}_{Q}^{G_{P} Q}\left(\operatorname{Res}_{G_{P} Q}^{G}\left(\sigma^{i}\right) u\right) \sigma_{Q}^{i} v
$$

Thus $\operatorname{Br}_{Q}^{G_{P} Q}(u) v=v$ and $(P, u) \triangleleft(Q, v)$. The argument is reversible, and so (C4) holds.

Let $P$ be a cyclic subgroup of $D$. The diagram

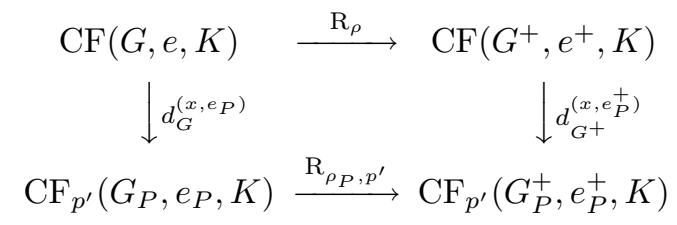

is commutative for all generators $x$ of $P$. Indeed, let $\chi \in(G, e)^{\vee}$ and $x^{\prime} \in G_{P, p^{\prime}}^{+}$. Then

$$
\begin{aligned}
& d_{G^{+}}^{\left(x, e_{P}^{+}\right)}\left(\operatorname{Res}_{G^{+}}^{G}(\chi)\right)\left(x^{\prime}\right)=\chi\left(x x^{\prime} e_{P}^{+}\right), \\
& \operatorname{Res}_{G_{P}^{+}}^{G_{P}}\left(d_{G}^{\left(x, e_{P}\right)}(\chi)\right)\left(x^{\prime}\right)=\chi\left(x x^{\prime} e_{P}\right) .
\end{aligned}
$$

The commutativity now follows since $\chi\left(x x^{\prime} e_{P}^{+}\right)=\sum_{i} \chi\left(x x^{\prime} \sigma_{P}^{i} e_{P}\right)=\chi\left(x x^{\prime} e_{P}\right)$.

Corollary (1E). Suppose $\langle\sigma\rangle$ acts regularly on the orbit of $e$ and $D$ is a defect group of e. Let $H=N_{G}(D)$ and $H^{+}=N_{G^{+}}(D)$, and let $f$ and $f^{+}$be the Brauer 
correspondents of e and $e^{+}$in $\mathcal{O H}$ and $\mathrm{OH}^{+}$. Then $e$ and $f$ are isotypic blocks of $\mathcal{O} G$ and $\mathcal{O} H$ if and only if $e^{+}$and $f^{+}$are isotypic blocks of $\mathcal{O G}^{+}$and $\mathcal{O H}^{+}$.

Proof. We have $\operatorname{Br}_{D}^{G}\left(\sigma^{i} e\right)=\sigma_{D}^{i} \operatorname{Br}_{D}^{G}(e)=\sigma_{D}^{i} f$ for $1 \leq i \leq t$. In particular, $\operatorname{Res}_{H}^{G}(\sigma)$ acts regularly on the orbit of $f, \sigma_{D}^{i} f$ is the Brauer correspondent of $\sigma^{i} e$, and $f^{+}=\sum_{i=1}^{t} \sigma_{D}^{i} f$. Now $e$ and $e^{+}$are isotypic blocks by (1D); $f$ and $f^{+}$are also isotypic blocks by (1D). Thus (1E) holds.

\section{2. +Equivariance}

Let $G$ be a finite group, let $G^{+}$be a subgroup of index 2 , and let $\operatorname{sgn}_{G}$ be the sign character of $G$ with kernel $G^{+}$. We write $\chi \mapsto \chi^{*}$ and $e \mapsto e^{*}$ for the action of $\operatorname{sgn}_{G}$ on the irreducible characters and blocks of $G$. Let $e$ and $e^{+}$be blocks of $\mathcal{O} G$ and $\mathcal{O G}^{+}$. The configuration $\left(G, G^{+}, e, e^{+}\right)$is a +configuration if $e^{+}$is the unique block of $\mathcal{O} G^{+}$covered by $e$ and if $e$ is the unique block of $\mathcal{O} G$ covering $e^{+}$. This is equivalent to the condition $e=e^{+}$or to the conditions $e^{+}$is stable under $G$ and $e^{*}=e$. Let $\left(H, H^{+}, f, f^{+}\right)$be a second + configuration with corresponding sign character $\operatorname{sgn}_{H}$, and write $\psi \mapsto \psi^{*}$ and $f \mapsto f^{*}$ for the action of $\operatorname{sgn}_{H}$ on the irreducible characters and blocks of $H$. An isometry $\mu=(\mathrm{J}, \epsilon)$ in $\mathcal{R}_{K}((G, e),(H, f))$ is +equivariant with respect to $\left(G^{+}, H^{+}\right)$if

$$
\mathrm{J}\left(\chi^{*}\right)=\mathrm{J}(\chi)^{*}, \quad \epsilon\left(\chi^{*}\right)=\epsilon(\chi)
$$

for all $\chi \in(G, e)^{\vee}$.

Lemma (2A). Let $\left(G, G^{+}, e, e^{+}\right)$and $\left(H, H^{+}, f, f^{+}\right)$be + configurations, and let $\mu$ be a +equivariant isometry in $\mathcal{R}_{K}((G, e),(H, f))$.

(i) $\chi \in(G, e)^{\vee}$ is reducible on $G^{+}$if and only if $J(\chi) \in(H, f)^{\vee}$ is reducible on $\mathrm{H}^{+}$.

(ii) $\mu(g, h)=0$ if $\operatorname{sgn}_{G}(g) \neq \operatorname{sgn}_{H}(h)$.

Proof. (i) holds since $\operatorname{Res}_{G^{+}}^{G}(\chi)$ and $\operatorname{Res}_{H^{+}}^{H}(\mathrm{~J}(\chi))$ are reducible if and only if $\chi^{*}=\chi$ and $\mathrm{J}(\chi)^{*}=\mathrm{J}(\chi)$. In (ii) either $g \notin G^{+}$or $h \notin H^{+}$. Now $\mu(g, h)$ is a sum of terms $\epsilon(\chi) \chi(g) \mathrm{J}(\chi)\left(h^{-1}\right)$. If $\operatorname{Res}_{G^{+}}^{G}(\chi)$ and $\operatorname{Res}_{H^{+}}^{H}(\mathrm{~J}(\chi))$ are reducible, then one of $\chi(g)$ or $\mathrm{J}(\chi)\left(h^{-1}\right)$ is zero and $\epsilon(\chi) \chi(g) \mathrm{J}(\chi)\left(h^{-1}\right)=0$. If $\operatorname{Res}_{G^{+}}^{G}(\chi)$ and $\operatorname{Res}_{H^{+}}^{H}(\mathrm{~J}(\chi))$ are irreducible, then $\chi \neq \chi^{*}, \mathrm{~J}(\chi) \neq \mathrm{J}(\chi)^{*}$, and

$$
\begin{aligned}
& \epsilon(\chi) \chi(g) \mathrm{J}(\chi)\left(h^{-1}\right)+\epsilon\left(\chi^{*}\right) \chi^{*}(g) \mathrm{J}\left(\chi^{*}\right)\left(h^{-1}\right) \\
& \quad=\epsilon(\chi) \chi(g) \mathrm{J}(\chi)\left(h^{-1}\right)\left(1+\operatorname{sgn}_{G}(g) \operatorname{sgn}_{H}(h)\right)
\end{aligned}
$$

is zero. Thus $\mu(g, h)$ is a sum of zero subsums and $\mu(g, h)=0$.

Let $\mu=(\mathrm{J}, \epsilon)$ be a +equivariant isometry in $\mathcal{R}_{K}((G, e),(H, f))$. An isometry $\mu^{+}=\left(\mathrm{J}^{+}, \epsilon^{+}\right)$in $\mathcal{R}_{K}\left(\left(G^{+}, e^{+}\right),\left(H^{+}, f^{+}\right)\right)$is $\mu$-induced if one and hence both of the following diagrams are commutative:

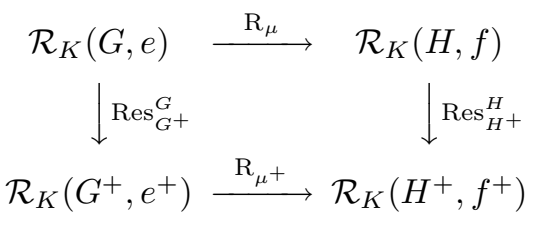




$$
\begin{array}{rrr}
\mathcal{R}_{K}\left(G^{+}, e^{+}\right) & \stackrel{\mathrm{R}_{\mu^{+}}}{\longrightarrow} & \mathcal{R}_{K}\left(H^{+}, f^{+}\right) \\
\downarrow \operatorname{Ind}_{G^{+}}^{G} & & \\
\mathcal{R}_{K}(G, e) & \stackrel{\mathrm{R}_{\mu}}{\longrightarrow} & \mathcal{R}_{K}(H, f)
\end{array}
$$

All $\mu$-induced isometries $\mu^{+}=\left(\mathrm{J}^{+}, \epsilon^{+}\right)$arise as follows: Let

$$
\left(G^{+}, e^{+}\right)^{\vee}=\left(G^{+}, e^{+}\right)_{e}^{\vee} \cup\left(G^{+}, e^{+}\right)_{n}^{\vee},
$$

where the characters in $\left(G^{+}, e^{+}\right)_{e}^{\vee}$ extend to $G$ and the characters in $\left(G^{+}, e^{+}\right)_{n}^{\vee}$ do not extend to $G$. If $\chi^{+} \in\left(G^{+}, e^{+}\right)_{e}^{\vee}$ and $\chi^{+}=\operatorname{Res}_{G^{+}}^{G}(\chi)$ for $\chi \in(G, e)^{\vee}$, let

$$
\mathrm{J}^{+}\left(\chi^{+}\right)=\operatorname{Res}_{H^{+}}^{H}(\mathrm{~J}(\chi)), \quad \epsilon^{+}\left(\chi^{+}\right)=\epsilon(\chi) .
$$

$\mathrm{J}^{+}\left(\chi^{+}\right)$and $\epsilon^{+}\left(\chi^{+}\right)$are well-defined since

$$
\operatorname{Res}_{H^{+}}^{H}\left(\mathrm{~J}\left(\chi^{*}\right)\right)=\operatorname{Res}_{H^{+}}^{H}\left(\mathrm{~J}(\chi)^{*}\right)=\operatorname{Res}_{H^{+}}^{H}(\mathrm{~J}(\chi))
$$

and $\epsilon\left(\chi^{*}\right)=\epsilon(\chi)$. If $\chi_{+}$and $\chi_{-}$are characters in $\left(G^{+}, e^{+}\right)_{n}^{\vee}$ such that $\chi_{+}+\chi_{-}=$ $\operatorname{Res}_{G^{+}}^{G}(\chi)$ for $\chi \in(G, e)^{\vee}$, let $\operatorname{Res}_{H^{+}}^{H}(\mathrm{~J}(\chi))=\mathrm{J}(\chi)_{+}+\mathrm{J}(\chi)_{-}$and set

$$
\mathrm{J}^{+}\left(\chi_{ \pm}\right)=\mathrm{J}(\chi)_{ \pm}, \quad \epsilon^{+}\left(\chi_{ \pm}\right)=\epsilon(\chi) .
$$

$\mathrm{J}^{+}\left(\chi_{ \pm}\right)$depends on the labeling of the constituents $\chi_{ \pm}$and $\mathrm{J}(\chi)_{ \pm}$. In all cases $\chi$ covers $\chi^{+}$if and only if $\mathrm{J}(\chi)$ covers $\mathrm{J}^{+}\left(\chi^{+}\right)$. Moreover, $\mathrm{J}^{+}\left(\left(\chi^{+}\right)^{g}\right)=\mathrm{J}^{+}\left(\chi^{+}\right)^{h}$ and $\epsilon^{+}\left(\left(\chi^{+}\right)^{g}\right)=\epsilon^{+}\left(\chi^{+}\right)$for $g \in G-G^{+}$and $h \in H-H^{+}$.

Lemma (2B). Let $\left(G, G^{+}, e, e^{+}\right)$and $\left(H, H^{+}, f, f^{+}\right)$be configurations, let $\mu$ be a + equivariant isometry in $\mathcal{R}_{K}((G, e),(H, f))$, and let $\mu^{+}=\left(\mathrm{J}^{+}, \epsilon^{+}\right)$be a $\mu$-induced isometry in $\mathcal{R}_{K}\left(\left(G^{+}, e^{+}\right),\left(H^{+}, f^{+}\right)\right)$. Suppose $p$ is odd and $\mu$ is perfect. Then $\mu^{+}$ is perfect if and only if the generalized character of $G^{+} \times H^{+}$defined by

$$
\Delta\left(\mu^{+}\right)(g, h)=\sum_{\left\{\chi_{+}, \chi_{-}\right\} \subseteq\left(G^{+}, e^{+}\right)_{n}^{\vee}} \epsilon^{+}\left(\chi_{ \pm}\right)\left(\chi_{+}-\chi_{-}\right)(g)\left(\mathrm{J}^{+}\left(\chi_{+}\right)-\mathrm{J}^{+}\left(\chi_{-}\right)\right)\left(h^{-1}\right)
$$

is perfect.

Proof. If $(g, h) \in G^{+} \times H^{+}$, then

$$
\begin{aligned}
\mu(g, h)= & \sum_{\chi \in(G, e)^{\vee}} \epsilon(\chi) \chi(g) \mathrm{J}(\chi)\left(h^{-1}\right) \\
= & \sum_{\chi^{+} \in\left(G^{+}, e^{+}\right)_{e}^{\vee}} 2 \epsilon^{+}\left(\chi^{+}\right) \chi^{+}(g) \mathrm{J}^{+}\left(\chi^{+}\right)\left(h^{-1}\right) \\
& \quad+\sum_{\left\{\chi_{+}, \chi_{-}\right\} \subseteq\left(G^{+}, e^{+}\right)_{n}^{\vee}} \epsilon^{+}\left(\chi_{ \pm}\right)\left(\chi_{+}+\chi_{-}\right)(g)\left(\mathrm{J}^{+}\left(\chi_{+}\right)+\mathrm{J}^{+}\left(\chi_{-}\right)\right)\left(h^{-1}\right) \\
& \quad 2 \mu^{+}(g, h)-\sum_{\left\{\chi_{+}, \chi_{-}\right\} \subseteq\left(G^{+}, e^{+}\right)_{n}^{\vee}} \epsilon^{+}\left(\chi_{ \pm}\right)\left(\chi_{+}-\chi_{-}\right)(g)\left(\mathrm{J}^{+}\left(\chi_{+}\right)-\mathrm{J}^{+}\left(\chi_{-}\right)\right)\left(h^{-1}\right) .
\end{aligned}
$$

Thus

$$
\operatorname{Res}_{G^{+} \times H^{+}}^{G \times H}(\mu)=2 \mu^{+}-\Delta\left(\mu^{+}\right)
$$

and (2B) follows. 
Remark (2C). If $\left(\chi_{+}-\chi_{-}\right)(g)\left(\mathrm{J}^{+}\left(\chi_{+}\right)-\mathrm{J}^{+}\left(\chi_{-}\right)\right)\left(h^{-1}\right)$ is perfect for all $\left\{\chi_{+}, \chi_{-}\right\} \subseteq$ $\left(G^{+}, e^{+}\right)_{n}^{\vee}$, then all $\mu$-induced isometries $\mu^{+}$are perfect. We note $\Delta\left(\mu^{+}\right)(g, h) \neq 0$ only if $g$ and $h$ lie in conjugacy classes of $G$ and $H$ which split in $G^{+}$and $H^{+}$.

Suppose $G_{0}$ and $G_{1}$ are finite groups, $G_{0}^{+}$and $G_{1}^{+}$are normal subgroups of $G_{0}$ and $G_{1}$ of index 2 , and $\operatorname{sgn}_{G_{0}}$ and $\operatorname{sgn}_{G_{1}}$ are the corresponding sign characters. Let $G=G_{0} \times G_{1}$, and let $\operatorname{sgn}_{G}=\operatorname{sgn}_{G_{0}} \times \operatorname{sgn}_{G_{1}}$ be the sign character of $G$ with kernel

$$
G^{+}=\left(G_{0} \times G_{1}\right)^{+}=\left\{\left(g_{0}, g_{1}\right) \in G_{0} \times G_{1}: \operatorname{sgn}_{G_{0}}\left(g_{0}\right)=\operatorname{sgn}_{G_{1}}\left(g_{1}\right)\right\} .
$$

In addition, let $G^{++}=G_{0}^{+} \times G_{1}^{+}$and let $\operatorname{sgn}_{G^{+}}$be the sign character of $G^{+}$with respect to $G^{++}$, that is,

$$
\operatorname{sgn}_{G^{+}}=\operatorname{Res}_{G^{+}}^{G}\left(\operatorname{sgn}_{G_{0}} \times 1_{G_{1}}\right)=\operatorname{Res}_{G^{+}}^{G}\left(1_{G_{0}} \times \operatorname{sgn}_{G_{1}}\right) .
$$

A character $\chi$ in $G^{\vee}$ with components $\chi_{i} \in G_{i}^{\vee}$ is reducible on $G^{+}$if and only if each $\chi_{i}$ is reducible on $G_{i}^{+}$. In particular, if $\chi$ is reducible on $G^{+}$and $\operatorname{Res}_{G^{+}}^{G}(\chi)=\chi_{+}+$ $\chi_{-}$, then $\operatorname{Res}_{G^{++}}^{G^{+}}\left(\chi_{+}\right)$and $\operatorname{Res}_{G^{++}}^{G^{+}}\left(\chi_{-}\right)$are also reducible and all three characters $\chi, \chi_{+}, \chi_{-}$vanish off $G^{++}$.

Proposition (2D). Let $\left(G_{0}, G_{0}^{+}, e_{0}, e_{0}^{+}\right)$and $\left(G_{1}, G_{1}^{+}, e_{1}, e_{1}^{+}\right)$be + configurations, let $e$ be the block $e_{0} e_{1}$ of $\mathcal{O} G$, and let $e^{++}$be the block $e_{0}^{+} e_{1}^{+}$of $\mathcal{O} G^{++}$. Then there exists a unique block $e^{+}$of $\mathcal{O} G^{+}$covered by e. Moreover, $\left(G, G^{+}, e, e^{+}\right)$and $\left(G^{+}, G^{++}, e^{+}, e^{++}\right)$are + configurations.

Proof. Every block of $G^{+}$covered by $e$ covers $e^{++}$. The hypotheses imply that $\operatorname{sgn}_{G} e=e$ and $e^{++}$is stable under $G$. Suppose first that $\chi_{0}^{+} \chi_{1}^{+}$is a character in $\left(G^{++}, e^{++}\right)^{\vee}$ stable under $G$. Each factor $\chi_{i}^{+}$extends to two characters $\chi_{i}$ and $\chi_{i}^{*}$ in $\left(G_{i}, e_{i}\right)^{\vee}$, and the four characters $\chi_{0} \chi_{1}, \chi_{0}^{*} \chi_{1}^{*}, \chi_{0}^{*} \chi_{1}, \chi_{0} \chi_{1}^{*}$ in $(G, e)^{\vee}$ are distinct. The restrictions of these four characters to $G^{+}$are irreducible and so belong to a unique block $e^{+}$of $\mathcal{O} G^{+}$. Thus $e^{+}$is the unique block of $\mathcal{O} G^{+}$covered by $e$, and $\left(G, G^{+}, e, e^{+}\right)$is a +configuration. The two distinct characters of $G^{+}$extending $\chi_{0}^{+} \chi_{1}^{+}$are

$$
\begin{aligned}
& \chi^{+}=\operatorname{Res}_{G^{+}}^{G}\left(\chi_{0} \chi_{1}\right)=\operatorname{Res}_{G^{+}}^{G}\left(\chi_{0}^{*} \chi_{1}^{*}\right), \\
& \chi^{-}=\operatorname{Res}_{G^{+}}^{G}\left(\chi_{0} \chi_{1}^{*}\right)=\operatorname{Res}_{G^{+}}^{G}\left(\chi_{0}^{*} \chi_{1}\right),
\end{aligned}
$$

and $\chi^{-}=\operatorname{sgn}_{G^{+}} \chi^{+}$. Thus $\left(G^{+}, G^{++}, e^{+}, e^{++}\right)$is a +configuration and $\operatorname{sgn}_{G^{+}} e^{+}=$ $e^{+}$. Suppose next that $\chi_{0}^{+} \chi_{1}^{+}$is a character in $\left(G^{++}, e^{++}\right)^{\vee}$ not stable under $G$. Then $\chi_{0}^{+} \chi_{1}^{+}$is not stable under $G^{+}$and $\operatorname{Ind}_{G^{++}}^{G^{+}}\left(\chi_{0}^{+} \chi_{1}^{+}\right)$is irreducible. The block $e^{+}$of $\mathcal{O} G^{+}$containing $\operatorname{Ind}_{G^{+}}^{G^{+}}\left(\chi_{0}^{+} \chi_{1}^{+}\right)$is the unique block of $\mathcal{O} G^{+}$covering $e^{++}$ and $\operatorname{sgn}_{G^{+}} e^{+}=e^{+}$. Thus (2D) also holds in this case.

Let $\left(H_{0}, H_{0}^{+}, f_{0}, f_{0}^{+}\right)$and $\left(H_{1}, H_{1}^{+}, f_{1}, f_{1}^{+}\right)$be a second pair of +configurations, and let $H, H^{+}, H^{++}, \operatorname{sgn}_{H}, \operatorname{sgn}_{H^{+}}, f^{+}, f^{++}$have the analogous meanings for this pair. In particular, $\left(H, H^{+}, f, f^{+}\right)$and $\left(H^{+}, H^{++}, f^{+}, f^{++}\right)$are +configurations.

Proposition (2E). Let $\left(G_{i}, G_{i}^{+}, e_{i}, e_{i}^{+}\right)$and $\left(H_{i}, H_{i}^{+}, f_{i}, f_{i}^{+}\right)$be +configurations and let $\mu_{i}=\left(\mathrm{J}_{i}, \epsilon_{i}\right)$ be a +equivariant isometry in $\mathcal{R}_{K}\left(\left(G_{i}, e_{i}\right),\left(H_{i}, f_{i}\right)\right)$ for $i=0$, 1. Then the following hold:

(i) $\mu=\mu_{0} \times \mu_{1}$ is a +equivariant isometry in $\mathcal{R}_{K}((G, e),(H, f))$ with respect to $\left(G^{+}, H^{+}\right)$. 
(ii) All $\mu$-induced isometries $\mu^{+}$in $\mathcal{R}_{K}\left(\left(G^{+}, e^{+}\right),\left(H^{+}, f^{+}\right)\right)$are + equivariant with respect to $\left(G^{++}, H^{++}\right)$.

Proof. Let $\mu=(\mathrm{J}, \epsilon)$, where $\mathrm{J}=\mathrm{J}_{0} \times \mathrm{J}_{1}$ and $\epsilon=\epsilon_{0} \times \epsilon_{1}$. Let $\chi \in(G, e)^{\vee}$, where $\chi=\chi_{0} \chi_{1}$ and $\chi_{i} \in\left(G_{i}, e_{i}\right)^{\vee}$. Then

$$
\begin{aligned}
& \epsilon\left(\chi^{*}\right)=\epsilon_{0}\left(\chi_{0}^{*}\right) \epsilon_{1}\left(\chi_{1}^{*}\right)=\epsilon_{0}\left(\chi_{0}\right) \epsilon_{1}\left(\chi_{1}\right)=\epsilon(\chi), \\
& \mathrm{J}\left(\chi^{*}\right)=\mathrm{J}_{0}\left(\chi_{0}^{*}\right) \mathrm{J}_{1}\left(\chi_{1}^{*}\right)=\mathrm{J}_{0}\left(\chi_{0}\right)^{*} \mathrm{~J}_{1}\left(\chi_{1}\right)^{*}=\mathrm{J}(\chi)^{*},
\end{aligned}
$$

and (i) holds. We set $\left(\chi^{+}\right)^{\#}=\operatorname{sgn}_{G^{+}} \chi^{+}$and $\left(\psi^{+}\right)^{\#}=\operatorname{sgn}_{H^{+}} \psi^{+}$for $\chi^{+}$in $\left(G^{+}\right)^{\vee}$ and for $\psi^{+}$in $\left(H^{+}\right)^{\vee}$. Let $\mu^{+}=\left(\mathrm{J}^{+}, \epsilon^{+}\right)$be a $\mu$-induced isometry in $\mathcal{R}_{K}\left(\left(G^{+}, e^{+}\right),\left(H^{+}, f^{+}\right)\right)$. Let $\chi^{+} \in\left(G^{+}, e^{+}\right)^{\vee}$ and choose $\chi$ in $(G, e)^{\vee}$ covering $\chi^{+}$. Let $\chi=\chi_{0} \chi_{1}$, where $\chi_{i} \in\left(G_{i}, e_{i}\right)^{\vee}$. Then $\mathrm{J}(\chi)=\mathrm{J}_{0}\left(\chi_{0}\right) \mathrm{J}_{1}\left(\chi_{1}\right)$ is a character in $(H, f)^{\vee}$ covering $\mathrm{J}^{+}\left(\chi^{+}\right)$. If $\operatorname{Res}_{G^{++}}^{G^{+}}\left(\chi^{+}\right)$is reducible, then $\left(\chi^{+}\right)^{\#}=\chi^{+}$and $\operatorname{Res}_{G_{i}^{+}}^{G_{i}}\left(\chi_{i}\right)$ is reducible for some $i$. So $\operatorname{Res}_{H_{i}^{+}}^{H_{i}}\left(\mathrm{~J}_{i}\left(\chi_{i}\right)\right)$ is reducible for the same $i$ and $\operatorname{Res}_{H^{++}}^{H^{+}}\left(\mathrm{J}^{+}\left(\chi^{+}\right)\right)$is reducible. Thus $\epsilon^{+}\left(\left(\chi^{+}\right)^{\#}\right)=\epsilon^{+}\left(\chi^{+}\right)$and $\mathrm{J}^{+}\left(\left(\chi^{+}\right)^{\#}\right)=$ $\left(\mathrm{J}^{+}\left(\chi^{+}\right)\right)^{\#}$. If $\operatorname{Res}_{G^{++}}^{G^{+}}\left(\chi^{+}\right)$is irreducible, then $\chi^{+}=\operatorname{Res}_{G^{+}}^{G}(\chi)$ and

$$
\begin{aligned}
\chi^{+} & =\operatorname{Res}_{G^{+}}^{G}\left(\chi_{0} \chi_{1}\right)=\operatorname{Res}_{G^{+}}^{G}\left(\chi_{0}^{*} \chi_{1}^{*}\right), \\
\left(\chi^{+}\right)^{\#} & =\operatorname{Res}_{G^{+}}^{G}\left(\chi_{0} \chi_{1}^{*}\right)=\operatorname{Res}_{G^{+}}^{G}\left(\chi_{0}^{*} \chi_{1}\right) .
\end{aligned}
$$

Since $\mu_{0}$ and $\mu_{1}$ are +equivariant, $\epsilon^{+}\left(\left(\chi^{+}\right)^{\#}\right)=\epsilon^{+}\left(\chi^{+}\right)$and

$$
\begin{aligned}
\mathrm{J}^{+}\left(\left(\chi^{+}\right)^{\#}\right) & =\operatorname{Res}_{H^{+}}^{H}\left(\mathrm{~J}_{0}\left(\chi_{0}\right) \mathrm{J}_{1}\left(\chi_{1}^{*}\right)\right) \\
& =\operatorname{Res}_{H^{+}}^{H}\left(\mathrm{~J}_{0}\left(\chi_{0}\right) \mathrm{J}_{1}\left(\chi_{1}\right)^{*}\right) \\
& =\left(\operatorname{Res}_{H^{+}}^{H}\left(\mathrm{~J}_{0}\left(\chi_{0}\right) \mathrm{J}_{1}\left(\chi_{1}\right)\right)\right)^{\#} \\
& =\mathrm{J}^{+}\left(\chi^{+}\right)^{\#} .
\end{aligned}
$$

This completes the proof of (ii).

We continue with the situation of $(2 \mathrm{E})$. Suppose $\mu_{i}^{+}=\left(\mathrm{J}_{i}^{+}, \epsilon_{i}^{+}\right)$is a $\mu_{i}$-induced isometry in $\mathcal{R}_{K}\left(\left(G_{i}^{+}, e_{i}^{+}\right),\left(H_{i}^{+}, f_{i}^{+}\right)\right)$for $i=0,1$, and $\mu^{++}$is the isometry $\mu_{0}^{+} \times \mu_{1}^{+}$in $\mathcal{R}_{K}\left(\left(G^{++}, e^{++}\right),\left(H^{++}, f^{++}\right)\right)$. We may choose a $\mu$-induced isometry $\mu^{+}=\left(\mathrm{J}^{+}, \epsilon^{+}\right)$ such that $\mu^{++}$is $\mu^{+}$-induced. Indeed, fix notation so that $\mathrm{J}_{i}^{+}\left(\chi_{i, \pm}\right)=\psi_{i, \pm}$ whenever $\mathrm{J}_{i}\left(\chi_{i}\right)=\psi_{i}, \operatorname{Res}_{G_{i}^{+}}^{G_{i}}\left(\chi_{i}\right)=\chi_{i,+}+\chi_{i,-}$, and $\operatorname{Res}_{H_{i}^{+}}^{H_{i}}\left(\psi_{i}\right)=\psi_{i,+}+\psi_{i,-}$. Suppose $\mathrm{J}(\chi)=\psi$, where $\operatorname{Res}_{G^{+}}^{G}(\chi)=\chi_{+}+\chi_{-}$and $\operatorname{Res}_{H^{+}}^{H^{2}}(\psi)=\psi_{+}+\psi_{-}$are reducible. Then $\chi=\chi_{0} \chi_{1}$ and $\psi=\psi_{0} \psi_{1}$, where $\operatorname{Res}_{G_{i}^{+}}^{G_{i}}\left(\chi_{i}\right)=\chi_{i,+}+\chi_{i,-}$ and $\operatorname{Res}_{H_{i}^{+}}^{H_{i}}\left(\psi_{i}\right)=$ $\psi_{i,+}+\psi_{i,-}$ are reducible for $i=0,1$. We label $\chi_{ \pm}$and $\psi_{ \pm}$so that

$$
\begin{array}{r}
\operatorname{Res}_{G}^{G^{++}}\left(\chi_{+}\right)=\chi_{0,+} \chi_{1,+}+\chi_{0,-} \chi_{1,-}, \\
\operatorname{Res}_{G^{++}}^{G^{+}}\left(\chi_{-}\right)=\chi_{0,+} \chi_{1,-}+\chi_{0,-} \chi_{1,+}, \\
\operatorname{Res}_{H^{++}}^{H^{+}}\left(\psi_{+}\right)=\psi_{0,+} \psi_{1,+}+\psi_{0,-} \psi_{1,-}, \\
\operatorname{Res}_{H^{++}}^{H^{+}}\left(\psi_{-}\right)=\psi_{0,+} \psi_{1,-}+\psi_{0,-} \psi_{1,+},
\end{array}
$$


and choose $\mathrm{J}^{+}$so that $\mathrm{J}^{+}\left(\chi_{ \pm}\right)=\psi_{ \pm}$for all such $\chi$ and $\psi$. Then

$$
\begin{array}{rrr}
\mathcal{R}_{K}\left(G^{+}, e^{+}\right) & \stackrel{\mathrm{R}_{\mu^{+}}}{\longrightarrow} & \mathcal{R}_{K}\left(H^{+}, f^{+}\right) \\
\downarrow \operatorname{Res}_{G^{++}}^{G^{+}} & & \downarrow \operatorname{Res}_{H^{++}}^{++} \\
\mathcal{R}_{K}\left(G^{++}, e^{++}\right) & \stackrel{\mathrm{R}_{\mu++}}{\longrightarrow} & \mathcal{R}_{K}\left(H^{++}, f^{++}\right)
\end{array}
$$

is a commutative diagram. Thus $\mu^{+}$is $\mu$-induced and $\mu^{++}$is $\mu^{+}$-induced.

Proposition (2F). Suppose the hypotheses of (2E) hold and $\mu_{i}^{+}$is a $\mu_{i}$-induced isometry in $\mathcal{R}_{K}\left(\left(G_{i}^{+}, e_{i}^{+}\right),\left(H_{i}^{+}, f_{i}^{+}\right)\right)$for $i=0,1$.

(i) There exists a $\mu$-induced isometry $\mu^{+}$in $\mathcal{R}_{K}\left(\left(G^{+}, e^{+}\right),\left(H^{+}, f^{+}\right)\right)$such that $\mu^{++}=\mu_{0}^{+} \times \mu_{1}^{+}$is $\mu^{+}$-induced.

(ii) If $p$ is odd, $\mu_{i}^{+}$is perfect for $i=0,1$, and $\mu^{+}$is as in (i), then $\mu^{+}$is perfect.

Proof. (i) follows from the preceding paragraph. For (ii) it suffices by (2B) to prove that

$$
\Delta\left(\mu^{+}\right)(g, h)=\sum_{\left\{\chi_{+}, \chi_{-}\right\} \subseteq\left(G^{+}, e^{+}\right)_{n}^{\vee}} \epsilon^{+}\left(\chi_{ \pm}\right)\left(\chi_{+}-\chi_{-}\right)(g)\left(\psi_{+}-\psi_{-}\right)\left(h^{-1}\right)
$$

is perfect on its support $G^{++} \times H^{++}$. We may suppose $\mu^{+}$satisfies (2.4). Let $g_{0} \in G_{0}^{+}, g_{1} \in G_{1}^{+}, h_{0} \in H_{0}^{+}, h_{1} \in H_{1}^{+}$. Then

$$
\begin{aligned}
& \Delta\left(\mu^{+}\right)\left(g_{0} g_{1}, h_{0} h_{1}\right)=\left[\sum_{\substack{\left\{\chi_{0,+,} \chi_{0,-}\right\} \\
\subseteq\left(G_{0}, e_{0}\right)_{n}^{v}}} \epsilon_{0}^{+}\left(\chi_{0, \pm}\right)\left(\chi_{0,+}-\chi_{0,-}\right)\left(g_{0}\right)\left(\psi_{0,+}-\psi_{0,-}\right)\left(h_{0}^{-1}\right)\right] \\
& \cdot\left[\sum_{\substack{\left\{\chi_{1,+,}, \chi_{1,-}\right\} \\
\subseteq\left(G_{1}, e_{1}\right)_{n}^{v}}} \epsilon_{1}^{+}\left(\chi_{1, \pm}\right)\left(\chi_{1,+}-\chi_{1,-}\right)\left(g_{1}\right)\left(\psi_{1,+}-\psi_{1,-}\right)\left(h_{1}^{-1}\right)\right] .
\end{aligned}
$$

The bracketed sums are perfect generalized characters of $G_{0}^{+} \times H_{0}^{+}$and $G_{1}^{+} \times H_{1}^{+}$ since $\mu_{0}^{+}$and $\mu_{1}^{+}$are perfect. Thus $\Delta\left(\mu^{+}\right)$is perfect.

\section{The ISOTYPy CONJECTURE IN SYMmetriC GROUPS}

Let $G$ be the symmetric group $S(\Pi)$ on a set $\Pi$ of cardinality $n$. By the Nakayama conjecture a $p$-block $e$ of $\mathcal{O} G$ is parametrized by a $p$-core $\lambda_{0}$, and irreducible characters $\chi_{\lambda}$ in $(G, e)^{\vee}$ are parametrized by partitions $\lambda$ of $n$ with $p$-core $\lambda_{0}$. In particular, $n=n_{0}+p w$, where $n_{0}=\left|\lambda_{0}\right|$ and $w \geq 0$ is the so-called weight of $e$. We suppose that $e$ has an abelian defect group $D$, or equivalently, that $w<p$. Thus $\Pi=\Pi_{0} \cup \Pi_{1}$ is the disjoint union of sets $\Pi_{0}$ and $\Pi_{1}$ of cardinality $n_{0}$ and pw. We may suppose $\Pi_{1}=\Gamma \times \Omega$, where $\Gamma=\{1,2, \ldots, p\}$ and $\Omega$ is a set of $w$ elements. Let $X=S(\Gamma)$ and $Y=N_{X}(P)$, where $P$ is a fixed Sylow $p$-subgroup of $X$. In particular, $Y$ is a Frobenius group with kernel $P$ of order $p$ and a cyclic complement of order $p-1$.

We take $D$ as the Sylow $p$-subgroup $P^{\Omega}$ of $S\left(\Pi_{1}\right)$ and set $H=N_{G}(D)$, so that $H=H_{0} \times H_{1}$ with $H_{0}=S\left(\Pi_{0}\right)$ and $H_{1}=Y \nmid S(\Omega)$. We view $Y \nmid S(\Omega)$ as the product set $Y^{\Omega} \times S(\Omega)$, where $Y^{\Omega}$ is the set of functions from $\Omega$ to $Y, S(\Omega)$ acts 
on $Y^{\Omega}$ on the left by $\left({ }^{\sigma} f\right)(i)=f\left(\sigma^{-1}(i)\right)$ or $\left({ }^{\sigma} f\right)_{i}=f_{\sigma^{-1}(i)}$, and multiplication in $Y \curlyvee S(\Omega)$ is defined by

$$
(f, \sigma)(g, \tau)=\left(f\left({ }^{\sigma} g\right), \sigma \tau\right) .
$$

The Brauer correspondent $f$ of $e$ in $\mathcal{O} H$ has the form $f=f_{0} \times f_{1}$, where $f_{0}$ is the block of defect 0 of $\mathcal{O} H_{0}$ parametrized by $\lambda_{0}$ and $f_{1}$ is the principal block of $\mathcal{O} H_{1}$. We note that $f_{1}$ is the unique block of $\mathcal{O} H_{1}$, since $H_{1} / D$ is a $p^{\prime}$-group and $C_{H_{1}}(D)=D$.

Let $\lambda$ be a partition of $n$ with $p$-core $\lambda_{0}$ and $p$-quotient $\Lambda=\left(\lambda_{1}, \lambda_{2}, \ldots, \lambda_{p}\right)$. Then $\lambda_{0}$ can be gotten by removing a sequence $\left(\nu_{1}, \nu_{2}, \ldots, \nu_{w}\right)$ of $p$-hooks $\nu_{i}$ from $\lambda$. The sign $v(\lambda)=(-1)^{\sum_{1}^{w} \ell\left(\nu_{i}\right)}$, where $\ell\left(\nu_{i}\right)$ is the leg length of $\nu_{i}$, is independent of the choice of $\left(\nu_{1}, \nu_{2}, \ldots, \nu_{w}\right)$. The residue of the $(i, j)$-node in the Young diagram of $\lambda$ is the integer $r$ such that $0 \leq r<p$ and $r \equiv j-i(\bmod p)$. A hook of $\lambda$ has $p$-singular length if and only if the hand and foot node residues of the hook are respectively $k, k+1$ for some $k$ modulo $p$. We normalize $\Lambda$ so that $\lambda_{i}$ corresponds to hooks of $\lambda$ of $p$-singular length and hand node residue $i-1$. Let $\lambda^{*}$ be the dual partition of $\lambda$, let $\lambda_{0}^{*}$ be the $p$-core of $\lambda^{*}$, and let $\Lambda^{*}=\left(\lambda_{1}^{*}, \lambda_{2}^{*}, \ldots, \lambda_{p}^{*}\right)$ be the $p$-quotient of $\lambda^{*}$. Then $\lambda_{0}^{*}$ is the dual $\left(\lambda_{0}\right)^{*}$ of $\lambda_{0}$ and $\lambda_{\gamma}^{*}=\left(\lambda_{p+1-\gamma}\right)^{*}$ for $1 \leq \gamma \leq p$. In particular, $\lambda=\lambda^{*}$ if and only if $\lambda_{0}=\lambda_{0}^{*}$ and $\lambda_{\gamma}=\left(\lambda_{p+1-\gamma}\right)^{*}$ for $1 \leq \gamma \leq p$. Indeed, the $(i, j)$-hook of $\lambda$ and the $(j, i)$-hook of $\lambda^{*}$ have the same length. So if this length is $p$-singular, then the hand node residues of these hooks are of the form $k$ and $-k-1$ modulo $p$.

Let $Y^{\vee}=\left\{\xi_{\gamma}: 1 \leq \gamma \leq p\right\}$. The characters in $(H, f)^{\vee}$ have the form $\chi_{\lambda_{0}} \times \psi_{\Lambda}$, where $\left(H_{0}, f_{0}\right)^{\vee}=\left\{\chi_{\lambda_{0}}\right\}, \psi_{\Lambda} \in H_{1}^{\vee}$, and $\Lambda$ is a mapping

$$
Y^{\vee} \longrightarrow\{\text { partitions }\}, \quad \xi_{\gamma} \mapsto \lambda_{\gamma},
$$

such that $\sum_{\gamma}\left|\lambda_{\gamma}\right|=w$. We also represent $\Lambda$ by the $p$-tuple $\left(\lambda_{1}, \lambda_{2}, \ldots, \lambda_{p}\right)$. We recall the definition of $\psi_{\Lambda}$. Let $\Omega=\coprod_{\gamma=1}^{p} \Omega_{\gamma}^{\Lambda}$ be a partition where $\left|\Omega_{\gamma}^{\Lambda}\right|=\left|\lambda_{\gamma}\right|$, and let $\xi_{\Lambda} \in\left(Y^{\Omega}\right)^{\vee}$ be such that $\left(\xi_{\Lambda}\right)_{i}=\xi_{\gamma}$ for $i \in \Omega_{\gamma}^{\Lambda}$. The stabilizer $H_{1, \Lambda}$ of $\xi_{\Lambda}$ in $H_{1}$ is $Y^{\Omega} S\left(\Omega^{\Lambda}\right)$, where

$$
S\left(\Omega^{\Lambda}\right)=S\left(\Omega_{1}^{\Lambda}\right) \times S\left(\Omega_{2}^{\Lambda}\right) \times \cdots \times S\left(\Omega_{p}^{\Lambda}\right),
$$

and $\xi_{\Lambda}$ extends to a character $\mathrm{E}\left(\xi_{\Lambda}\right)$ of $H_{1, \Lambda}$. Let $\omega_{\Lambda}=\omega_{\lambda_{1}} \times \omega_{\lambda_{2}} \times \cdots \times \omega_{\lambda_{p}}$ be the irreducible character of $S\left(\Omega^{\Lambda}\right)$, where $\omega_{\lambda_{\gamma}}$ is the character of $S\left(\Omega_{\gamma}^{\Lambda}\right)$ parametrized by $\lambda_{\gamma}$. We may view $\omega_{\Lambda}$ as a character of $H_{1, \Lambda}$ by inflation. Then

$$
\psi_{\Lambda}=\operatorname{Ind}_{H_{1, \Lambda}}^{H_{1}}\left(\mathrm{E}\left(\xi_{\Lambda}\right) \omega_{\Lambda}\right)
$$

We give a formula for $\mathrm{E}\left(\xi_{\Lambda}\right)(f, \sigma)$ for $f \in Y^{\Omega}$ and $\sigma \in S\left(\Omega^{\Lambda}\right)$. Write $\sigma=$ $\sigma_{1} \sigma_{2} \cdots \sigma_{d}$ as a product of disjoint cycles in $S(\Omega)$ and write

$$
(f, \sigma)=\left(f_{1}, \sigma_{1}\right)\left(f_{2}, \sigma_{2}\right) \cdots\left(f_{d}, \sigma_{d}\right),
$$

where $f_{\delta} \in Y^{\Omega_{\delta}^{\sigma}}$ and $\Omega_{\delta}^{\sigma}$ is the support of $\sigma_{\delta}$. Let $n_{\delta}=\left|\Omega_{\delta}^{\sigma}\right|$, let $i_{\delta} \in \Omega_{\delta}^{\sigma}$, and let

$$
p_{i_{\delta}}(f, \sigma)=f\left(i_{\delta}\right) f\left(\sigma^{-1}\left(i_{\delta}\right)\right) \cdots f\left(\sigma^{-\left(n_{\delta}-1\right)}\left(i_{\delta}\right)\right) .
$$

In particular, $\left(f_{\delta}, \sigma_{\delta}\right)^{n_{\delta}}=\left(p_{i_{\delta}}(f, \sigma), 1\right)$. Moreover

$$
\begin{aligned}
p_{i_{\delta}}\left({ }^{h}(f, \sigma)\right) & =h_{i_{\delta}} p_{i_{\delta}}(f, \sigma) h_{i_{\delta}}^{-1}, \\
p_{g\left(i_{\delta}\right)}\left({ }^{g}(f, \sigma)\right) & =p_{i_{\delta}}(f, \sigma)
\end{aligned}
$$


for $h \in Y^{\Omega}$ and $g \in S(\Omega)$. Since $(f, \sigma) \in H_{1, \Lambda}, \sigma$ stabilizes each $\Omega_{\gamma}^{\Lambda}$. Let $\Delta_{\gamma}$ be index sets such that $\Omega_{\gamma}^{\Lambda}=\underset{\delta \in \Delta_{\gamma}}{\coprod} \Omega_{\delta}^{\sigma}$ is the orbit decomposition of $\sigma$ on $\Omega_{\gamma}^{\Lambda}$. Then

$$
\mathrm{E}\left(\xi_{\Lambda}\right)(f, \sigma)=\prod_{\gamma} \prod_{\delta \in \Delta_{\gamma}} \xi_{\gamma}\left(p_{i_{\delta}}(f, \sigma)\right)
$$

(see $[12, \S 4.3])$. We call $\mathrm{E}\left(\xi_{\Lambda}\right)$ the James-Kerber extension of $\xi_{\Lambda}$.

We give a Murnaghan-Nakayama type formula for $\psi_{\Lambda}(f, \sigma)$ for the preceding element $(f, \sigma)$. We fix $s$ with $1 \leq s \leq d$ and consider pairs $(\underline{\zeta}, \underline{\gamma})$ of sequences $\underline{\zeta}=$ $\left(\zeta_{1}, \zeta_{2}, \ldots, \zeta_{s}\right)$ and $\underline{\gamma}=\left(\gamma_{1}, \gamma_{2}, \ldots, \gamma_{s}\right)$ with the following properties: $\zeta_{1}$ is a hook of length $\left|\sigma_{1}\right|$ of the $\gamma_{1}$-component of $\Lambda ; \zeta_{2}$ is a hook of length $\left|\sigma_{2}\right|$ of the $\gamma_{2}$-component of $\left(\zeta_{1}, \gamma_{1}\right) * \Lambda$, the $p$-tuple gotten from $\Lambda$ by removing $\zeta_{1}$ from the $\gamma_{1}$-component of $\Lambda ; \zeta_{3}$ is a hook of length $\left|\sigma_{3}\right|$ of the $\gamma_{3}$-component of $\left(\zeta_{2}, \gamma_{2}\right) *\left(\zeta_{1}, \gamma_{1}\right) * \Lambda$, the $p$-tuple gotten from $\left(\zeta_{1}, \gamma_{1}\right) * \Lambda$ by removing $\zeta_{2}$ from the $\gamma_{2}$-component of $\left(\zeta_{1}, \gamma_{1}\right) * \Lambda$; and so on. Set

$$
(\underline{\zeta}, \underline{\gamma}) * \Lambda=\left(\zeta_{s}, \gamma_{s}\right) * \cdots *\left(\zeta_{2}, \gamma_{2}\right) *\left(\zeta_{1}, \gamma_{1}\right) * \Lambda
$$

and $v(\underline{\zeta})=(-1)^{\sum_{1}^{s} \ell\left(\zeta_{k}\right)}$.

Proposition (3A). With the above notation

$$
\psi_{\Lambda}(f, \sigma)=\sum_{(\underline{\zeta}, \underline{\gamma})} v(\underline{\zeta}) \prod_{k=1}^{s} \xi_{\gamma_{k}}\left(p_{i_{k}}(f, \sigma)\right) \psi_{(\underline{\zeta}, \underline{\gamma}) * \Lambda}\left(f^{\prime}, \sigma^{\prime}\right),
$$

where $\left(f^{\prime}, \sigma^{\prime}\right)=\left(f_{s+1}, \sigma_{s+1}\right) \cdots\left(f_{d}, \sigma_{d}\right)$ and $(\underline{\zeta}, \underline{\gamma})$ runs over all pairs of the above type.

Proof. We consider first the case $s=1$. By definition

$$
\begin{aligned}
\psi_{\Lambda}(f, \sigma) & =\operatorname{Ind}_{H_{1, \Lambda}}^{H_{1}}\left(\mathrm{E}\left(\xi_{\Lambda}\right) \omega_{\Lambda}\right)(f, \sigma) \\
& =\sum_{g \in S\left(\Omega^{\Lambda}\right) \backslash S(\Omega)}\left(\mathrm{E}\left(\xi_{\Lambda}\right) \omega_{\Lambda}\right)\left({ }^{g}(f, \sigma)\right),
\end{aligned}
$$

since $H_{1}=Y^{\Omega} S(\Omega)$ and $H_{1, \Lambda}=Y^{\Omega} S\left(\Omega^{\Lambda}\right)$. We identify $S\left(\Omega^{\prime}\right)$ for $\Omega^{\prime} \subseteq \Omega$ with the subgroup of $S(\Omega)$ fixing $\Omega-\Omega^{\prime}$ elementwise. Let $\Omega^{\prime}=\Omega-\Omega_{1}^{\sigma}$ and decompose

$$
S(\Omega)=\bigcup_{g \in \Gamma} S\left(\Omega^{\Lambda}\right) g S\left(\Omega^{\prime}\right)
$$

as a disjoint union of double cosets modulo $\left(S\left(\Omega^{\Lambda}\right), S\left(\Omega^{\prime}\right)\right)$. We say that a representative $g$ in $\Gamma$ is good and that the double coset $S\left(\Omega^{\Lambda}\right) g S\left(\Omega^{\prime}\right)$ is good if $g\left(\Omega_{1}^{\sigma}\right) \subseteq \Omega_{\gamma}^{\Lambda}$ for some $\gamma$. This definition is independent of the choice of coset representative $g$, since $S\left(\Omega^{\prime}\right)$ fixes $\Omega_{1}^{\sigma}$ elementwise and $S\left(\Omega^{\Lambda}\right)$ stabilizes each $\Omega_{\gamma}^{\Lambda}$. Moreover, the integer $\gamma$ such that $g\left(\Omega_{1}^{\sigma}\right) \subseteq \Omega_{\gamma}^{\Lambda}$ depends only on the double coset. Let $\Gamma^{\prime}$ be the subset of good representatives $g$ in $\Gamma$. If $g$ and $g^{\prime}$ are in $\Gamma^{\prime}$ and $g\left(\Omega_{1}^{\sigma}\right)$ and $g^{\prime}\left(\Omega_{1}^{\sigma}\right)$ are contained in the same $\Omega_{\gamma}^{\Lambda}$, then $g=g^{\prime}$. Indeed, there exists $x \in S\left(\Omega^{\Lambda}\right)$ such that $x(g(i))=g^{\prime}(i)$ for all $i \in \Omega_{1}^{\sigma}$, so $g^{-1} x^{-1} g^{\prime} \in S\left(\Omega^{\prime}\right)$ and $g^{\prime} \in S\left(\Omega^{\Lambda}\right) g S\left(\Omega^{\prime}\right)$. Thus there is a bijection between good double cosets and components $\lambda_{\gamma}$ of $\Lambda$ such that $\left|\lambda_{\gamma}\right| \geq\left|\sigma_{1}\right|$.

Given $g$ in $\Gamma$, let $S\left(\Omega^{\Lambda}\right) g S\left(\Omega^{\prime}\right)=\bigcup_{t \in T_{g}} S\left(\Omega^{\Lambda}\right) g t$, where $T_{g}$ is a set of representatives $t$ for the cosets $\left(g^{-1} S\left(\Omega^{\Lambda}\right) g \cap S\left(\Omega^{\prime}\right)\right) \backslash S\left(\Omega^{\prime}\right)$. If $g \in \Gamma^{\prime}$ and $g\left(\Omega_{1}^{\sigma}\right) \subseteq \Omega_{\gamma_{1}}^{\Lambda}$, 
then

$$
\begin{aligned}
g^{-1} S\left(\Omega^{\Lambda}\right) g \cap S\left(\Omega^{\prime}\right) & =S\left(g^{-1}\left(\Omega^{\Lambda}\right)\right) \cap S\left(\Omega^{\prime}\right) \\
& =S\left(g^{-1}\left(\Omega_{\gamma_{1}}^{\Lambda}\right)-\Omega_{1}^{\sigma}\right) \times \prod_{\gamma \neq \gamma_{1}} S\left(g^{-1}\left(\Omega_{\gamma}^{\Lambda}\right)\right) .
\end{aligned}
$$

For $g \in \Gamma^{\prime}$ we say $t$ in $T_{g}$ and the coset $S\left(\Omega^{\Lambda}\right) g t$ are good if ${ }^{g t}(f, \sigma) \in H_{1, \Lambda}$. Thus $t$ is good if and only if for each $\delta=2,3, \ldots, d$, there is a $\gamma$ such that $g t\left(\Omega_{\delta}^{\sigma}\right) \subseteq \Omega_{\gamma}^{\Lambda}$. This definition is independent of the choice of representative $t$. Let $T_{g}^{\prime}$ be the subset of good representatives $t$ in $T_{g}$. It follows that

$$
\psi_{\Lambda}(f, \sigma)=\sum_{g \in \Gamma^{\prime}} \sum_{t \in T_{g}^{\prime}}\left(\mathrm{E}\left(\xi_{\Lambda}\right) \omega_{\Lambda}\right)\left(^{g t}(f, \sigma)\right)
$$

since $\mathrm{E}\left(\xi_{\Lambda}\right)$ and $\omega_{\Lambda}$ are zero by convention on arguments not in $H_{1, \Lambda}$. To prove (3.3) for the case $s=1$, it suffices to show that

$$
\sum_{t \in T_{g}^{\prime}}\left(\mathrm{E}\left(\xi_{\Lambda}\right) \omega_{\Lambda}\right)\left({ }^{g t}(f, \sigma)\right)=\sum_{\zeta_{1}} v\left(\zeta_{1}\right) \xi_{\gamma_{1}}\left(p_{i_{1}}(f, \sigma)\right) \psi_{\left(\zeta_{1}, \gamma_{1}\right) * \Lambda}\left(f^{\prime}, \sigma^{\prime}\right),
$$

where $\zeta_{1}$ runs over all hooks of length $\left|\sigma_{1}\right|$ in $\lambda_{\gamma_{1}}$.

Given $g \in \Gamma^{\prime}$ such that $g\left(\Omega_{1}^{\sigma}\right) \subseteq \Omega_{\gamma_{1}}^{\Lambda}$ and given $t$ in $T_{g}^{\prime}$, let $\Delta_{g t, \gamma}$ be the index sets such that

$$
\Omega_{\gamma}^{\Lambda}=\bigcup_{\delta \in \Delta_{g t, \gamma}} g t\left(\Omega_{\delta}^{\sigma}\right)
$$

Let

$$
\Delta_{g t, \gamma}^{\prime}= \begin{cases}\Delta_{g t, \gamma} & \text { if } \gamma \neq \gamma_{1}, \\ \Delta_{g t, \gamma}-\{1\} & \text { if } \gamma=\gamma_{1} .\end{cases}
$$

Then

$$
\begin{aligned}
\left.\mathrm{E}\left(\xi_{\Lambda}\right){ }^{g t}(f, \sigma)\right) & =\prod_{\gamma} \prod_{\delta \in \Delta_{g t, \gamma}} \xi_{\gamma}\left(p_{g t\left(i_{\delta}\right)}\left({ }^{g t}(f, \sigma)\right)\right) \\
& =\xi_{\gamma_{1}}\left(p_{g t\left(i_{1}\right)}\left({ }^{g t}\left(f_{1}, \sigma_{1}\right)\right)\right)\left[\prod_{\gamma} \prod_{\delta \in \Delta_{g t, \gamma}^{\prime}} \xi_{\gamma}\left(p_{g t\left(i_{\delta}\right)}\left({ }^{g t}\left(f^{\prime}, \sigma^{\prime}\right)\right)\right)\right] \\
& =\xi_{\gamma_{1}}\left(p_{i_{1}}\left(f_{1}, \sigma_{1}\right)\right)\left[\prod_{\gamma} \prod_{\delta \in \Delta_{g t, \gamma}^{\prime}} \xi_{\gamma}\left(p_{g t\left(i_{\delta}\right)}\left({ }^{g t}\left(f^{\prime}, \sigma^{\prime}\right)\right)\right)\right] .
\end{aligned}
$$

The usual Murnaghan-Nakayama formula (see [12, 21.1]) gives

$$
\begin{aligned}
\left.\omega_{\Lambda}{ }^{g t} \sigma\right) & =\prod_{\gamma} \omega_{\lambda_{\gamma}}\left(\prod_{\delta \in \Delta_{g t, \gamma}}{ }^{g t}\left(\sigma_{\delta}\right)\right) \\
& =\omega_{\lambda_{\gamma_{1}}}\left(\prod_{\delta \in \Delta_{g t, \gamma_{1}}}{ }^{g t}\left(\sigma_{\delta}\right)\right) \prod_{\gamma \neq \gamma_{1}} \omega_{\lambda_{\gamma}}\left(\prod_{\delta \in \Delta_{g t, \gamma}}{ }^{g t}\left(\sigma_{\delta}\right)\right) \\
& =\sum_{\zeta_{1}} v\left(\zeta_{1}\right) \omega_{\zeta_{1} * \lambda_{\gamma_{1}}}\left(\prod_{\delta \in \Delta_{g t, \gamma_{1}}^{\prime}}{ }^{g t}\left(\sigma_{\delta}\right)\right) \prod_{\gamma \neq \gamma_{1}} \omega_{\lambda_{\gamma}}\left(\prod_{\delta \in \Delta_{g t, \gamma}}{ }^{g t}\left(\sigma_{\delta}\right)\right) \\
& \left.=\sum_{\zeta_{1}} v\left(\zeta_{1}\right) \omega_{\left(\zeta_{1}, \gamma_{1}\right) * \Lambda}{ }^{g t} \sigma^{\prime}\right),
\end{aligned}
$$


where $\zeta_{1}$ runs over all hooks of length $\left|\sigma_{1}\right|$ in $\lambda_{\gamma_{1}}$. Thus

$$
\begin{aligned}
\sum_{t \in T_{g}^{\prime}}\left(\mathrm{E}\left(\xi_{\Lambda}\right) \omega_{\Lambda}\right) & \left.{ }^{g t}(f, \sigma)\right)=\sum_{\zeta_{1}} v\left(\zeta_{1}\right) \xi_{\gamma_{1}}\left(p_{i_{1}}\left(f_{1}, \sigma_{1}\right)\right) \\
\times & \times \sum_{t \in T_{g}^{\prime}}\left[\prod_{\gamma} \prod_{\delta \in \Delta_{g t, \gamma}^{\prime}} \xi_{\gamma}\left(p_{g t\left(i_{\delta}\right)}\left({ }^{g t}\left(f^{\prime}, \sigma^{\prime}\right)\right)\right)\right] \omega_{\left(\zeta_{1}, \gamma_{1}\right) * \Lambda}\left({ }^{g t} \sigma^{\prime}\right) .
\end{aligned}
$$

It suffices to show that if $\zeta_{1}$ is a fixed hook in $\lambda_{\gamma_{1}}$ of length $\left|\sigma_{1}\right|$, then

$$
\left.\psi_{\left(\zeta_{1}, \gamma_{1}\right) * \Lambda}\left(f^{\prime}, \sigma^{\prime}\right)=\sum_{t \in T_{g}^{\prime}}\left[\prod_{\gamma} \prod_{\delta \in \Delta_{g t, \gamma}^{\prime}} \xi_{\gamma}\left(p_{g t\left(i_{\delta}\right)}\left({ }^{g t}\left(f^{\prime}, \sigma^{\prime}\right)\right)\right)\right] \omega_{\left(\zeta_{1}, \gamma_{1}\right) * \Lambda}{ }^{g t} \sigma^{\prime}\right) .
$$

Now $\left(\zeta_{1}, \gamma_{1}\right) * \Lambda$ corresponds to the $p$-tuple $\Lambda^{\prime}=\left(\lambda_{1}^{\prime}, \lambda_{2}^{\prime}, \ldots, \lambda_{p}^{\prime}\right)$, where

$$
\lambda_{\gamma}^{\prime}= \begin{cases}\lambda_{\gamma} & \text { if } \gamma \neq \gamma_{1}, \\ \zeta_{1} * \lambda_{\gamma} & \text { if } \gamma=\gamma_{1} .\end{cases}
$$

Let $\psi_{\Lambda^{\prime}}$ be the irreducible character of $Y 2 S\left(\Omega^{\prime}\right)$ parametrized by $\Lambda^{\prime}$. We use notation analogous to that for $\psi_{\Lambda}$. Namely, let $\Omega^{\prime}=\bigcup_{\gamma}{\Omega^{\prime}}_{\gamma}^{\Lambda^{\prime}}$, where

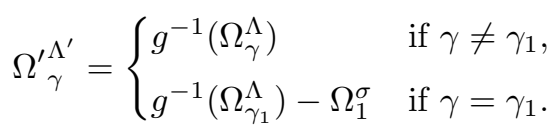

So $\lambda_{\gamma}^{\prime}$ is a partition of $\left|{\Omega^{\prime}}_{\gamma}^{\prime \Lambda^{\prime}}\right|$. Let $\xi_{\Lambda^{\prime}} \in\left(Y^{\Omega^{\prime}}\right)^{\vee}$ be such that $\left(\xi_{\Lambda^{\prime}}\right)_{i}=\xi_{\gamma}$ for $i \in{\Omega^{\prime}}_{\gamma}^{\Lambda^{\prime}}$. Then the stabilizer of $\xi_{\Lambda^{\prime}}$ in $Y^{\Omega^{\prime}} S\left(\Omega^{\prime}\right)$ is $Y^{\Omega^{\prime}} S\left({\Omega^{\prime}}^{\Lambda^{\prime}}\right)$, where

$$
S\left({\Omega^{\prime}}^{\Lambda^{\prime}}\right)=S\left({\Omega^{\prime}}_{1}^{\Lambda^{\prime}}\right) \times S\left({\Omega^{\prime}}_{2}^{\Lambda^{\prime}}\right) \times \cdots \times S\left({\Omega^{\prime \Lambda^{\prime}}}_{p}\right) .
$$

In particular, $T_{g}^{\prime}$ is a set of coset representatives of $S\left({\Omega^{\prime}}^{\prime}\right)$ in $S\left(\Omega^{\prime}\right)$ by (3.4). Let $\omega_{\Lambda^{\prime}}=\omega_{\lambda_{1}^{\prime}} \times \omega_{\lambda_{2}^{\prime}} \times \cdots \times \omega_{\lambda_{p}^{\prime}}$ be the irreducible character of $S\left({\Omega^{\prime}}^{\Lambda^{\prime}}\right)$, where $\omega_{\lambda_{\gamma}^{\prime}}$ is the irreducible character of $S\left({\Omega^{\prime}}_{\gamma}^{\Lambda^{\prime}}\right)$ parametrized by $\lambda_{\gamma}^{\prime}$. Let $\mathrm{E}\left(\xi_{\Lambda^{\prime}}\right)$ be the JamesKerber extension of $\xi_{\Lambda^{\prime}}$ to $Y^{\Omega^{\prime}} S\left({\Omega^{\prime}}^{\Lambda^{\prime}}\right)$. Then

$$
\begin{aligned}
\psi_{\left(\zeta_{1}, \gamma_{1}\right) * \Lambda}\left(f^{\prime}, \sigma^{\prime}\right) & =\psi_{\Lambda^{\prime}}\left(f^{\prime}, \sigma^{\prime}\right) \\
& =\operatorname{Ind}_{Y^{\Omega^{\prime}} S\left(\Omega^{\prime \Lambda^{\prime}}\right)}^{Y^{\prime}} S\left(\mathrm{E}\left(\xi_{\Lambda^{\prime}}\right) \omega_{\Lambda^{\prime}}\right)\left(f^{\prime}, \sigma^{\prime}\right) \\
& =\sum_{t \in T_{g}^{\prime}}\left(\mathrm{E}\left(\xi_{\Lambda^{\prime}}\right) \omega_{\Lambda^{\prime}}\right)\left({ }^{t}\left(f^{\prime}, \sigma^{\prime}\right)\right) .
\end{aligned}
$$

We can now show (3.7). Indeed, ${ }^{t}\left(f^{\prime}, \sigma^{\prime}\right) \in S\left({\Omega^{\prime}}^{\Lambda^{\prime}}\right)$ if and only if for each $\delta \geq 2$, there exists a $\gamma$ such that $t\left(\Omega_{\delta}^{\sigma}\right) \subseteq{\Omega^{\prime}}_{\gamma}^{\Lambda^{\prime}}$, or equivalently, such that $g t\left(\Omega_{\delta}^{\sigma}\right) \subseteq \Omega_{\gamma}^{\Lambda}$. Moreover, ${\Omega^{\prime}}_{\gamma}^{\Lambda^{\prime}}=\coprod_{\delta \in \Delta_{g t, \gamma}^{\prime}} t\left(\Omega_{\delta}^{\sigma}\right)$, since $\Omega_{\gamma}^{\Lambda}=\bigsqcup_{\delta \in \Delta_{g t, \gamma}} g t\left(\Omega_{\delta}^{\sigma}\right)$. Thus

$$
\begin{aligned}
\mathrm{E}\left(\xi_{\Lambda^{\prime}}\right)\left({ }^{t}\left(f^{\prime}, \sigma^{\prime}\right)\right) & =\prod_{\gamma} \prod_{\delta \in \Delta_{g t, \gamma}^{\prime}} \xi_{\gamma}\left(p_{t\left(i_{\delta}\right)}\left({ }^{t}\left(f^{\prime}, \sigma^{\prime}\right)\right)\right) \\
& =\prod_{\gamma} \prod_{\delta \in \Delta_{g t, \gamma}^{\prime}} \xi_{\gamma}\left(p_{g t\left(i_{\delta}\right)}\left({ }^{g t}\left(f^{\prime}, \sigma^{\prime}\right)\right)\right)
\end{aligned}
$$


and

$$
\begin{aligned}
\omega_{\Lambda^{\prime}}\left({ }^{t}\left(\sigma^{\prime}\right)\right) & =\prod_{\gamma} \omega_{\lambda_{\gamma}^{\prime}}\left(\prod_{\delta \in \Delta_{g t, \gamma}^{\prime}}{ }^{t}\left(\sigma_{\delta}\right)\right) \\
& =\omega_{\lambda_{\gamma_{1}}}\left(\prod_{\delta \in \Delta_{g t, \gamma_{1}}^{\prime}}{ }^{g t}\left(\sigma_{\delta}\right)\right) \prod_{\gamma \neq \gamma_{1}} \omega_{\lambda_{\gamma}}\left(\prod_{\delta \in \Delta_{g t, \gamma}}{ }^{g t}\left(\sigma_{\delta}\right)\right) \\
& =\omega_{\Lambda^{\prime}}\left({ }^{g t}\left(\sigma^{\prime}\right)\right) .
\end{aligned}
$$

This completes the proof of (3.3) for the case $s=1$. The general case of (3.3) now follows by iteration.

Remark. Formula (3.3) does not depend on the structure of $Y$. Equivalent formulas can also be found in [14, Théorème 2.8] and in [15, Theorem 4.3].

Definition. Let $A(\Gamma)$ be the alternating group on $\Gamma$ and label $\xi_{\gamma}$ in $Y^{\vee}$ so that $\xi_{\gamma}$ and $\xi_{p+1-\gamma}$ have the same restriction to $Y^{+}=Y \cap A(\Gamma)$ for $1 \leq \gamma \leq p$. In particular, $\xi_{\gamma}(1)=1$ for $\gamma \neq p^{*}$ and $\xi_{\gamma}(1)=p-1$ for $\gamma=p^{*}$, where $p^{*}=\frac{1}{2}(p+1)$. The Rouquier isometry $\mu=(\mathrm{J}, \epsilon)$ in $\mathcal{R}_{K}((G, e),(H, f))$ is defined as follows: For each partition $\lambda$ of $n$ with $p$-core $\lambda_{0}$ and $p$-quotient $\Lambda=\left(\lambda_{1}, \lambda_{2}, \ldots, \lambda_{p}\right)$, let

$$
\begin{array}{lll}
\mathrm{J}\left(\chi_{\lambda}\right)=\chi_{\lambda_{0}} \times \psi_{\Lambda}, & \epsilon\left(\chi_{\lambda}\right)=1 & \text { if } p=2, \\
\mathrm{~J}\left(\chi_{\lambda}\right)=\chi_{\lambda_{0}} \times \psi_{\widehat{\Lambda}}, & \epsilon\left(\chi_{\lambda}\right)=v(\lambda)(-1)^{\left|\lambda_{p^{*}}\right|} & \text { if } p>2,
\end{array}
$$

where $\widehat{\Lambda}$ is the $p$-tuple gotten from $\Lambda$ by replacing $\lambda_{p^{*}}$ by $\left(\lambda_{p^{*}}\right)^{*}$.

We define a Rouquier isometry in a more general context. An element $x=(f, 1)$ of $D$ is called standard if the non-identity values of $f$ are all equal. For a standard element $x$ let $\Omega=\Omega_{x, 0} \cup \Omega_{x, 1}$, where $x$ acts trivially on $\Gamma \times \Omega_{x, 0}$ and fixed-pointfreely on $\Gamma \times \Omega_{x, 1}$. Thus

$$
G_{x}=G_{x, 0} \times G_{x, 1}, \quad H_{x}=H_{x, 0} \times H_{x, 1},
$$

where $G_{x, 0}=S\left(\Pi_{0} \cup\left(\Gamma \times \Omega_{x, 0}\right)\right), H_{x, 0}=S\left(\Pi_{0}\right) \times\left(Y \backslash S\left(\Omega_{x, 0}\right)\right)$, and $G_{x, 1}=H_{x, 1}=$ $P \imath S\left(\Omega_{x, 1}\right)$. Let $H_{x, 0}=H_{x, 00} \times H_{x, 01}$, where $H_{x, 00}=S\left(\Pi_{0}\right)$ and $H_{x, 01}=Y 2 S\left(\Omega_{x, 0}\right)$. Let

$$
e_{x}=e_{x, 0} \times e_{x, 1}, \quad f_{x}=f_{x, 00} \times f_{x, 01} \times f_{x, 1},
$$

where $e_{x, 0}$ is the block of $\mathcal{O} G_{x, 0}$ parametrized by $\lambda_{0}, e_{x, 1}$ is the 1-block of $\mathcal{O} G_{x, 1}$, $f_{x, 00}$ is the block of $\mathcal{O} H_{x, 00}$ parametrized by $\lambda_{0}, f_{x, 01}$ is the 1-block of $\mathcal{O} H_{x, 01}$, and $f_{x, 1}$ is the 1-block of $\mathcal{O} H_{x, 1}$. In particular, $e_{x, 1}, f_{x, 01}$, and $f_{x, 1}$ are the unique blocks of $\mathcal{O} G_{x, 1}, \mathcal{O} H_{x, 01}$, and $\mathcal{O} H_{x, 1}$. Let $f_{x, 0}$ be the block $f_{x, 00} \times f_{x, 01}$ of $\mathcal{O} H_{x, 0}$. Then $e_{x, 0}$ and $f_{x, 0}$ have defect group $P^{\Omega_{x, 0}}$ and $f_{x, 0}$ is the Brauer correspondent of $e_{x, 0}$. Let $\mu_{x, 0}=\left(\mathrm{J}_{x, 0}, \epsilon_{x, 0}\right)$ be the Rouquier isometry in $\mathcal{R}_{K}\left(\left(G_{x, 0}, e_{x, 0}\right),\left(H_{x, 0}, f_{x, 0}\right)\right)$. Let $\mu_{x, 1}=\left(\mathrm{J}_{x, 1}, \epsilon_{x, 1}\right)$ be the identity isometry in $\mathcal{R}_{K}\left(\left(G_{x, 1}, 1\right),\left(H_{x, 1}, 1\right)\right)$. Let $\mu_{x}=\left(\mathrm{J}_{x}, \epsilon_{x}\right)$ be the isometry $\mu_{x, 0} \times \mu_{x, 1}$ in $\mathcal{R}_{K}\left(\left(G_{x}, e_{x}\right),\left(H_{x}, f_{x}\right)\right)$. If $\mu_{x, 0}$ is perfect, then $\mu_{x}$ is perfect We call $\mu_{x}$ the Rouquier isometry in $\mathcal{R}_{K}\left(\left(G_{x}, e_{x}\right),\left(H_{x}, f_{x}\right)\right)$. We note that $\mu_{x}$ depends only on $\langle x\rangle$ and not on the generator $x$.

For each $j=0,1,2, \ldots, w$ let $x_{j}$ be a standard element of $D$ such that $\left|\Omega_{x_{j}, 1}\right|=j$. The $w+1$ subgroups $\left\langle x_{j}\right\rangle$ then form a set of representatives for the $N_{G}\left(D, e_{D}\right)$-orbits of cyclic subgroups of $D$, since $N_{G}\left(D, e_{D}\right) \supseteq(Y \nmid S(\Omega))$.

Theorem (3B). e and $f$ are isotypic blocks of $\mathcal{O} G$ and $\mathcal{O} H$ with local system given by the translates of the Rouquier isometries $\mu_{x}$ for $x$ in $\left\{x_{0}, x_{1}, \ldots, x_{w}\right\}$. 
Proof. Condition (I1) holds by [1, Proposition 4.2] since $D$ is abelian. The blocks $e$ and $f$ are of principal type by a theorem of Marichal and Puig (see [3, (2.2)]). Let $x \in\left\{x_{1}, x_{2}, \ldots, x_{w}\right\}$. We prove the Rouquier isometries $\mu_{x}$ are perfect by induction on $|D|$. The claim is clear if $|D|=1$, so we may suppose $D>1$. By induction $\mu_{x, 0}$ and hence $\mu_{x}$ are perfect isometries. We claim the following diagram is commutative:

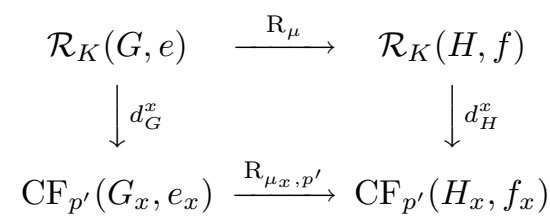

Suppose $p=2$. Then $w=1, G_{x}=H_{x}=S\left(\Pi_{0}\right) \times\langle x\rangle$, and $\lambda$ is a partition with 2core $\lambda_{0}$ and 2-quotient $(1,0)$ or $(0,1)$. The ordinary Murnaghan-Nakayama formula implies the commutativity of (3.9). Suppose $p>2$. We consider the top-right portion of (3.9). Let $\chi_{\lambda} \in(G, e)^{\vee}$, let $y \in H_{x, p^{\prime}}$, and write $y=y_{00} y_{01} y_{1}$, where $y_{00} \in H_{x, 00}, y_{01} \in H_{x, 01}$, and $y_{1} \in H_{x, 1}$. Let $x y_{1}=\left(f_{1}, \sigma_{1}\right)\left(f_{2}, \sigma_{2}\right) \cdots\left(f_{s}, \sigma_{s}\right)$, where the $\sigma_{i}$ are disjoint cycles of $S\left(\Omega_{x, 1}\right)$. Then

$$
\begin{aligned}
d_{H}^{x}\left(\mathrm{R}_{\mu}\left(\chi_{\lambda}\right)\right)(y) & =\epsilon\left(\chi_{\lambda}\right)\left(\chi_{\lambda_{0}} \times \psi_{\widehat{\Lambda}}\right)(x y) \\
& =\epsilon\left(\chi_{\lambda}\right) \chi_{\lambda_{0}}\left(y_{00}\right) \psi_{\widehat{\Lambda}}\left(y_{01}\left(x y_{1}\right)\right) \\
& =\epsilon\left(\chi_{\lambda}\right) \chi_{\lambda_{0}}\left(y_{00}\right) \sum_{(\underline{\zeta}, \underline{\gamma})} v(\underline{\zeta}) \prod_{k=1}^{s} \xi_{\gamma_{k}}\left(p_{i_{k}}\left(f_{k}, \sigma_{k}\right)\right) \psi_{(\underline{\zeta}, \underline{\gamma}) * \hat{\Lambda}}\left(y_{01}\right)
\end{aligned}
$$

by $(3 \mathrm{~A})$. For each pair $(\underline{\zeta}, \underline{\gamma})$ in the formula, let $M(\underline{\gamma})=\left\{k: \gamma_{k}=p^{*}\right\}$. Then

$$
d_{H}^{x}\left(\mathrm{R}_{\mu}\left(\chi_{\lambda}\right)\right)(y)=\epsilon\left(\chi_{\lambda}\right) \chi_{\lambda_{0}}\left(y_{00}\right) \sum_{(\underline{\zeta}, \underline{\gamma})} v(\underline{\zeta})(-1)^{|M(\underline{\gamma})|} \psi_{(\underline{\zeta}, \underline{\gamma}) * \widehat{\Lambda}}\left(y_{01}\right)
$$

since $p_{i_{k}}\left(f_{k}, \sigma_{k}\right) \in P^{\#}$, so that $\xi_{\gamma_{k}}\left(p_{i_{k}}\left(f_{k}, \sigma_{k}\right)\right)=1$ or -1 according as $\gamma_{k} \neq p^{*}$ or $\gamma_{k}=p^{*}$.

We consider the left-bottom portion of (3.9). Let $g \in G_{x, p^{\prime}}$ and write $g=g_{0} g_{1}$, where $g_{0} \in G_{x, 0, p^{\prime}}, g_{1} \in G_{x, 1, p^{\prime}}$. Each $p^{\prime}$-conjugacy class of $G_{x, 1}$ contains a unique conjugacy class of $S\left(\Omega_{x, 1}\right)$, since $S\left(\Omega_{x, 1}\right)$ is a Hall $p$-complement in $G_{x, 1}$. We may suppose without loss of generality that $g_{1}=(1, \sigma)$ for some $\sigma$ in $S\left(\Omega_{x, 1}\right)$. Let $\sigma=\sigma_{1} \sigma_{2} \cdots \sigma_{s}$ be the cycle decomposition of $\sigma$ in $S\left(\Omega_{x, 1}\right)$. The MurnaghanNakayama formula implies that

$$
\begin{aligned}
d_{G}^{x}\left(\chi_{\lambda}\right)(g) & =\chi_{\lambda}(x g) \\
& =\chi_{\lambda}\left(g_{0}\left(x g_{1}\right)\right) \\
& =\sum_{\underline{\nu}} v(\underline{\nu}) \chi_{\underline{\nu} * \lambda}\left(g_{0}\right),
\end{aligned}
$$

where $\underline{\nu}$ runs over sequences $\left(\nu_{1}, \nu_{2}, \ldots, \nu_{s}\right)$ of hooks $\nu_{k}$ of length $p\left|\sigma_{k}\right|$ of $\lambda$. Let $\tau$ run over representatives of the $p^{\prime}$-conjugacy classes of $S\left(\Omega_{x, 1}\right)$, let $\eta_{\tau}$ be the characteristic function of the $G_{x, 1}$-conjugacy class of $\tau$, and set

$$
\alpha_{\lambda}=\sum_{\underline{\nu}, \tau} v(\underline{\nu})\left(\chi_{\underline{\nu} * \lambda} \times \eta_{\tau}\right) .
$$


Then

$$
\alpha_{\lambda}\left(g_{0} g_{1}\right)= \begin{cases}\sum_{\underline{\nu}} v(\underline{\nu}) \chi_{\underline{\underline{\nu}} * \lambda}\left(g_{0}\right) & \text { if } g_{1} \in G_{x, 1, p^{\prime}}, \\ 0 & \text { if } g_{1} \notin G_{x, 1, p^{\prime}} .\end{cases}
$$

for $g_{0} \in G_{x, 0}$ and $g_{1} \in G_{x, 1}$. Thus $d_{G}^{x}\left(\chi_{\lambda}\right)=d_{G_{x}}^{1}\left(\alpha_{\lambda}\right)$ and

$$
\begin{aligned}
\mathrm{R}_{\mu_{x}, p^{\prime}}\left(d_{G}^{x}\left(\chi_{\lambda}\right)\right) & =\mathrm{R}_{\mu_{x}, p^{\prime}}\left(d_{G_{x}}^{1}\left(\alpha_{\lambda}\right)\right) \\
& =d_{H_{x}}^{1}\left(\mathrm{R}_{\mu_{x}}\left(\alpha_{\lambda}\right)\right),
\end{aligned}
$$

where the second equality holds since $\mu_{x}$ is perfect by induction. Now

$$
\begin{aligned}
\mathrm{R}_{\mu_{x}}\left(\alpha_{\lambda}\right) & =\sum_{\underline{\nu}, \tau} v(\underline{\nu})\left(\mathrm{R}_{\mu_{x, 0}}\left(\chi_{\underline{\nu} * \lambda}\right) \times \mathrm{R}_{\mu_{x, 1}}\left(\eta_{\tau}\right)\right) \\
& =\sum_{\underline{\nu}, \tau} v(\underline{\nu}) \epsilon_{x, 0}\left(\chi_{\underline{\nu} * \lambda}\right)\left(\chi_{\lambda_{0}} \times \psi_{(\underline{\zeta}, \underline{\gamma}) * \hat{\Lambda}} \times \eta_{\tau}\right),
\end{aligned}
$$

where $(\underline{\zeta}, \underline{\gamma})$ is obtained from $\underline{\nu}$ as follows: First $\underline{\nu}$ determines a pair $(\underline{\tilde{\zeta}}, \underline{\gamma})$ of sequences $\left(\tilde{\zeta}_{1}, \tilde{\zeta}_{2}, \ldots, \tilde{\zeta}_{s}\right)$ and $\left(\gamma_{1}, \gamma_{2}, \ldots, \gamma_{s}\right)$, where $\tilde{\zeta}_{k}$ is a hook of length $\left|\sigma_{k}\right|$ of the $\gamma_{k}$-component of $\Lambda$. So $\underline{\nu}$ determines a pair $(\underline{\zeta}, \underline{\gamma})$ of sequences $\left(\zeta_{1}, \zeta_{2}, \ldots, \zeta_{s}\right)$ and $\left(\gamma_{1}, \gamma_{2}, \ldots, \gamma_{s}\right)$, where $\zeta_{k}$ is a hook of length $\left|\bar{\sigma}_{k}\right|$ of the $\gamma_{k}$-component of $\widehat{\Lambda}$, and $\zeta_{k}=\tilde{\zeta}_{k}$ or $\left(\tilde{\zeta}_{k}\right)^{*}$ according as $\gamma_{k} \neq p^{*}$ or $\gamma_{k}=p^{*}$. Thus

$$
\mathrm{R}_{\mu_{x}, p^{\prime}}\left(d_{G}^{x}\left(\chi_{\lambda}\right)\right)=\sum_{\underline{\nu}, \tau} v(\underline{\nu}) \epsilon_{x, 0}\left(\chi_{\underline{\nu} * \lambda}\right) d_{H_{x}}^{1}\left(\chi_{\lambda_{0}} \times \psi_{(\underline{\zeta}, \underline{\gamma}) * \widehat{\Lambda}} \times \eta_{\tau}\right) .
$$

If $y \in H_{x, p^{\prime}}$ and $y=y_{00} y_{01} y_{1}$, where $y_{00} \in H_{x, 00}, y_{01} \in H_{x, 01}$, and $y_{1} \in H_{x, 1}$, then

$$
\mathrm{R}_{\mu_{x}, p^{\prime}}\left(d_{G}^{x}\left(\chi_{\lambda}\right)\right)(y)=\sum_{\underline{\nu}} v(\underline{\nu}) \epsilon_{x, 0}\left(\chi_{\underline{\underline{\nu}} * \lambda}\right) \chi_{\lambda_{0}}\left(y_{00}\right) \psi_{(\underline{\zeta}, \underline{\gamma}) * \widehat{\Lambda}}\left(y_{01}\right) .
$$

The commutativity of the diagram (3.9) holds by (3.10) and (3.11) if

$$
\epsilon\left(\chi_{\lambda}\right) v(\underline{\zeta})(-1)^{|M(\underline{\gamma})|}=v(\underline{\nu}) \epsilon_{x, 0}\left(\chi_{\underline{\underline{\nu} * \lambda}}\right)
$$

for corresponding $(\underline{\zeta}, \underline{\gamma})$ and $\underline{\nu}$. Now

$$
\begin{aligned}
\epsilon\left(\chi_{\lambda}\right) & =v(\lambda)(-1)^{\left|\lambda_{p^{*}}\right|}, \\
\epsilon_{x, 0}\left(\chi_{\underline{\underline{\nu} * \lambda}}\right) & =v(\underline{\nu} * \lambda)(-1)^{\left|(\underline{\nu} * \lambda)_{p^{*}}\right|} .
\end{aligned}
$$

So (3.12) becomes

$$
v(\lambda)(-1)^{\left|\lambda_{p^{*}}\right|} v(\underline{\zeta})(-1)^{|M(\underline{\gamma})|}=v(\underline{\nu}) v(\underline{\nu} * \lambda)(-1)^{\mid(\underline{\nu} * \lambda)_{p^{*} \mid}} .
$$

Since $\left|(\underline{\nu} * \lambda)_{p^{*}}\right|=\left|\lambda_{p^{*}}\right|-\sum_{k \in M(\underline{\gamma})}\left|\zeta_{k}\right|,(3.12)$ is equivalent to

$$
\begin{aligned}
v(\lambda) v(\underline{\nu} * \lambda) & =v(\underline{\nu}) v(\underline{\zeta})(-1)^{\left|\lambda_{p^{*}}\right|}(-1)^{|M(\underline{\gamma})|}(-1)^{\mid\left(\underline{\nu * \lambda)_{p^{*}} \mid}\right.} \\
& =v(\underline{\nu}) v \underline{\zeta}) \prod_{k \in M(\underline{\gamma})}(-1)^{\left(\left|\zeta_{k}\right|-1\right)} .
\end{aligned}
$$

But $v(\lambda) v(\underline{\nu} * \lambda)=v(\underline{\tilde{\zeta}}) v(\underline{\nu})$ by $[8,(2.15)]$ or $[9$, Théorème 1.4, b)]. Since

$$
v(\underline{\zeta}) v(\underline{\tilde{\zeta}})=\prod_{k \in M(\underline{\gamma})}(-1)^{\left(\left|\zeta_{k}\right|-1\right)}
$$


(3.13) holds and (3.9) is commutative. This argument for the commutativity of (3.9) still applies if $x$ is replaced by any generator of $\langle x\rangle$, since the lower horizontal arrow in (3.9) depends only on $\langle x\rangle$. (3B) now follows by transport of structure, as remarked in $\S 1$.

\section{An analog of Frobenius's theorem}

Let $G$ and $G^{+}$be the symmetric group $S(\Pi)$ and the alternating group $A(\Pi)$ on $\Pi$. An irreducible character $\chi_{\lambda}$ of $G$ is reducible on $G^{+}$if and only if $\lambda$ is selfdual. For such $\lambda$ let $\eta(\lambda)$ be the partition of $n$ with parts $\eta_{11}(\lambda), \eta_{22}(\lambda), \ldots, \eta_{s s}(\lambda)$, where $\eta_{i i}(\lambda)$ is the hook length of the $(i, i)$-node of $\lambda$ and $s$ is the number of diagonal

nodes of $\lambda$. Since the $\eta_{i i}(\lambda)$ are distinct odd integers, the conjugacy class $\mathcal{C}_{\eta(\lambda)}$ of $G$ parametrized by $\eta(\lambda)$ lies in $G^{+}$and splits in $G^{+}$. The mapping $\lambda \mapsto \eta(\lambda)$ thus induces a bijection $\chi_{\lambda} \mapsto \mathcal{C}_{\eta(\lambda)}$ from irreducible characters $\chi_{\lambda}$ of $G$ which split on $G^{+}$to conjugacy classes $\mathcal{C}_{\eta(\lambda)}$ of $G$ which lie in $G^{+}$and split in $G^{+}$. Let $\operatorname{Res}_{G^{+}}^{G}\left(\chi_{\lambda}\right)=\chi_{\lambda,+}+\chi_{\lambda,-}$ and $\mathcal{C}_{\eta(\lambda)}=\mathcal{C}_{\eta(\lambda),+} \cup \mathcal{C}_{\eta(\lambda),-}$ be the corresponding decompositions. We use the convention that $\sqrt{a}=\sqrt{-a} i$ for negative real $a$, where $i$ is a fixed primitive 4-th root of unity in $K$. In addition, we write $\sqrt{ \pm \eta(\lambda)}$ for $\sqrt{ \pm \eta_{11}(\lambda) \eta_{22}(\lambda) \cdots \eta_{s s}(\lambda)}$. The following is Frobenius's Theorem [11, §3].

Theorem (4A). Suppose $\chi_{\lambda}$ is an irreducible character of $G$ which splits on $G^{+}$. Let $g \in G^{+}$. Then $\left(\chi_{\lambda,+}-\chi_{\lambda,-}\right)(g) \neq 0$ if and only if $g$ is in $\mathcal{C}_{\eta(\lambda)}$. Moreover, $\chi_{\lambda, \pm}$ and $\mathcal{C}_{\eta(\lambda), \pm}$ may be labeled so that

$$
\begin{aligned}
& \chi_{\lambda, \pm}(g)=\frac{1}{2}\left(\epsilon_{\lambda}+\sqrt{\epsilon_{\lambda} \eta(\lambda)}\right) \quad \text { if } g \in \mathcal{C}_{\eta(\lambda), \pm}, \\
& \chi_{\lambda, \pm}(g)=\frac{1}{2}\left(\epsilon_{\lambda}-\sqrt{\epsilon_{\lambda} \eta(\lambda)}\right) \quad \text { if } g \in \mathcal{C}_{\eta(\lambda), \mp},
\end{aligned}
$$

where $\epsilon_{\lambda}=(-1)^{\frac{1}{2}(n-s)}$.

We suppose for the rest of this section that $\Pi=\Gamma \times \Omega$, where $\Gamma=\{1,2, \ldots, p\}$ and $|\Omega|=w<p$. Let $X$ and $X^{+}$be the symmetric group $S(\Gamma)$ and the alternating group $A(\Gamma)$ on $\Gamma$; let $Y=N_{X}(P)$ and $Y^{+}=N_{X^{+}}(P)$, where $P$ is a fixed Sylow p-subgroup of $X$; and let $H=N_{G}(D)$ and $H^{+}=N_{G^{+}}(D)$, where $D$ is the abelian Sylow $p$-subgroup $P^{\Omega}$ of $G$. We denote the sign characters of $G, H, X, Y, S(\Omega)$ with respect to $G^{+}, H^{+}, X^{+}, Y^{+}, A(\Omega)$ by $\operatorname{sgn}_{G}, \operatorname{sgn}_{H}, \operatorname{sgn}_{X}, \operatorname{sgn}_{Y}, \operatorname{sgn}_{S(\Omega)}$. In particular, $\operatorname{sgn}_{H}=\operatorname{Res}_{H}^{G}\left(\operatorname{sgn}_{G}\right)$ and $\operatorname{sgn}_{Y}=\operatorname{Res}_{Y}^{X}\left(\operatorname{sgn}_{X}\right)$. If $(f, \sigma)$ is an element of $H=Y \imath S(\Omega)$ with $f \in Y^{\Omega}$ and $\sigma \in S(\Omega)$, then

$$
\operatorname{sgn}_{H}(f, \sigma)=\operatorname{sgn}_{S(\Omega)}(\sigma) \prod_{i \in \Omega} \operatorname{sgn}_{Y}(f(i)) .
$$

Thus $(f, \sigma)$ is in $H^{+}$if and only if $\operatorname{sgn}_{S(\Omega)}(\sigma)=\prod_{i \in \Omega} \operatorname{sgn}_{Y}(f(i))$. The next two results hold for any finite group $Y$ and a normal subgroup $Y^{+}$of $Y$.

Lemma (4B). Let $(f, \sigma) \in Y \imath S(\Omega)$ and let $\Omega=\coprod_{\delta=1}^{d} \Omega_{\delta}^{\sigma}$ be the orbit decomposition of $\sigma$ on $\Omega$. Then the following hold:

(i) $(f, \sigma)^{Y}\left(S(\Omega) \cap\left(Y^{+} \imath S(\Omega)\right) \neq \emptyset\right.$ if and only if $p_{i_{\delta}}(f, \sigma) \in Y^{+}$for all $\delta$.

(ii) Let $(f, \sigma) \in Y^{+} \imath S(\Omega)$ and $g \in Y^{\Omega}$. Then ${ }^{(g, 1)}(f, \sigma) \in\left(Y^{+} \imath S(\Omega)\right)$ if and only if $g$ is constant modulo $Y^{+}$on $\Omega_{\delta}^{\sigma}$ for $1 \leq \delta \leq d$. 
Proof. Part (i) follows from (3.1). For (ii) we note that ${ }^{(g, 1)}(f, \sigma) \in\left(Y^{+}\{S(\Omega))\right.$ if and only if $g(i) f(i)\left(g\left(\sigma^{-1}\right)(i)\right)^{-1} \in Y^{+}$for all $i \in \Omega$. Since $f \in\left(Y^{+}\right)^{\Omega}$, this is equivalent to saying $g(i)\left(g\left(\sigma^{-1}(i)\right)\right)^{-1} \in Y^{+}$for all $i \in \Omega$.

Lemma (4C). Let $\psi^{+}$be a character of $Y^{+}$, let $\psi=\operatorname{Ind}_{Y^{+}}^{Y}\left(\psi^{+}\right)$, and let $\mathrm{E}\left(\left(\psi^{+}\right)^{\Omega}\right)$ and $\mathrm{E}\left(\psi^{\Omega}\right)$ be the James-Kerber extensions of $\left(\psi^{+}\right)^{\Omega}$ and $\psi^{\Omega}$ to $Y^{+} \imath S(\Omega)$ and $Y \nmid S(\Omega)$. Then

$$
\mathrm{E}\left(\psi^{\Omega}\right)=\operatorname{Ind}_{Y+2 S(\Omega)}^{Y 2 S(\Omega)}\left(\mathrm{E}\left(\left(\psi^{+}\right)^{\Omega}\right)\right) .
$$

Proof. Let $(f, \sigma) \in(Y \backslash S(\Omega))$ and let $\Omega=\coprod_{\delta=1}^{d} \Omega_{\delta}^{\sigma}$ be the orbit decomposition of $\sigma$ on $\Omega$. If $(f, \sigma)^{Y \zeta S(\Omega)} \cap\left(Y^{+} \imath S(\Omega)\right)=\emptyset$, then $\operatorname{Ind}_{Y+\imath S(\Omega)}^{Y \zeta(\Omega)}\left(\mathrm{E}\left(\left(\psi^{+}\right)^{\Omega}\right)\right)(f, \sigma)=0$. On the other hand, by $(4 \mathrm{~B})$, (i) there exists $\delta$ such that $p_{i_{\delta}}(f, \sigma) \notin Y^{+}$, so that

$$
\mathrm{E}\left(\psi^{\Omega}\right)(f, \sigma)=\prod_{\delta} \psi\left(p_{i_{\delta}}(f, \sigma)\right)=0 .
$$

Thus (4C) holds in this case. We may then suppose that $(f, \sigma) \in\left(Y^{+} \imath S(\Omega)\right)$ and that $p_{i_{\delta}}(f, \sigma) \in Y^{+}$for all $\delta$. Let $T$ be a transversal for $Y^{+}$in $Y$. Then

$$
\begin{aligned}
\mathrm{E}\left(\psi^{\Omega}\right)(f, \sigma) & =\prod_{\delta} \psi\left(p_{i_{\delta}}(f, \sigma)\right) \\
& =\prod_{\delta} \sum_{t \in T} \psi^{+}\left(t p_{i_{\delta}}(f, \sigma) t^{-1}\right) \\
& =\sum_{\left(t_{\delta}\right) \in T^{d}} \prod_{\delta} \psi^{+}\left(t_{\delta} p_{i_{\delta}}(f, \sigma) t_{\delta}^{-1}\right) .
\end{aligned}
$$

$T^{\Omega}$ is a transversal for $\left(Y^{+}\right)^{\Omega}$ in $Y^{\Omega}$. Let $T_{\sigma}^{\Omega}$ be the subset of all $g \in T^{\Omega}$ such that $g$ is constant on $\Omega_{\delta}^{\sigma}$ for all $\delta$. We may identify such a $g$ with the $d$-tuple $\left(t_{\delta}\right)$, where $t_{\delta}=g\left(i_{\delta}\right)$. This gives a bijection of $T_{\sigma}^{\Omega}$ with $T^{d}$. Then (4B), (ii) and (3.1) imply that

$$
\begin{aligned}
\operatorname{Ind}_{Y^{+} L S(\Omega)}^{Y l(\Omega)}\left(\mathrm{E}\left(\left(\psi^{+}\right)^{\Omega}\right)\right)(f, \sigma) & =\sum_{g \in T_{\sigma}^{\Omega}} \mathrm{E}\left(\left(\psi^{+}\right)^{\Omega}\right)\left(g(f, \sigma) g^{-1}\right) \\
& =\sum_{\left(t_{\delta}\right) \in T^{d}} \prod_{\delta} \psi^{+}\left(t_{\delta} p_{i_{\delta}}(f, \sigma) t_{\delta}^{-1}\right),
\end{aligned}
$$

and $(4 \mathrm{C})$ holds.

$Y$ is a Frobenius group with kernel $P=\langle a\rangle$ of order $p$ and cyclic complement $\langle b\rangle$ of order $p-1$. The set $\mathcal{R}=\left\{a, b^{i}: 1 \leq i \leq p-1\right\}$ is a transversal for the conjugacy classes of $Y$; the classes contained in $Y^{+}$are represented by $a$ and $b^{2 i}$ for $1 \leq i \leq \frac{1}{2}(p-1)$. Of these only $a^{Y}$ splits in $Y^{+}$, say $a^{Y}=a_{+}^{Y^{+}} \cup a_{-}^{Y^{+}}$. We choose $\xi_{1}=\operatorname{sgn}_{Y}, \xi_{p}=1_{Y}$, and label the characters $\xi_{\gamma}$ in $Y^{\vee}$ so that $\xi_{p+1-\gamma}=\xi_{\gamma} \operatorname{sgn}_{Y}$ for $1 \leq \gamma \leq p$. We also write $\xi_{p+1-\gamma}$ as $\xi_{\gamma}^{*}$. In particular, $\operatorname{Res}_{Y^{+}}^{Y}\left(\xi_{p^{*}}\right)=\xi_{p^{*},+}+\xi_{p^{*},-}$, where $p^{*}=\frac{1}{2}(p+1)$. We label $\xi_{p^{*}, \pm}$ and $a_{ \pm}$so that

$$
\xi_{p^{*},+}(x)-\xi_{p^{*},-}(x)= \pm \sqrt{\epsilon_{p} p} \text { for } x \in\left(a_{ \pm}\right)^{Y^{+}}
$$

where $\epsilon_{p}=(-1)^{\frac{p-1}{2}}$. As in $\S 3$ we parametrize the irreducible characters $\psi_{\Lambda}$ of $H$ by mappings

$$
\Lambda: Y^{\vee} \longrightarrow\{\text { partitions }\}, \quad \xi_{\gamma} \mapsto \lambda_{\gamma}
$$


where $\sum_{\gamma}\left|\lambda_{\gamma}\right|=w$. We also represent $\Lambda$ by the $p$-tuple $\left(\lambda_{1}, \lambda_{2}, \ldots, \lambda_{p}\right)$. In particular, $\operatorname{sgn}_{H}=\psi_{\Lambda_{\mathrm{sgn}}}$, where $\Lambda_{\mathrm{sgn}}=\left(\left\{1^{w}\right\}, \emptyset, \emptyset, \ldots, \emptyset\right)$. Indeed, the stabilizer $H_{\Lambda_{\mathrm{sgn}}}$ of $\Lambda_{\mathrm{sgn}}$ is $H$, and

$$
\begin{aligned}
\mathrm{E}\left(\xi_{\Lambda_{\mathrm{sgn}}}\right)(f, \sigma) & =\prod_{\delta \in \Delta_{1}} \xi_{1}\left(p_{i_{\delta}}(f, \sigma)\right) \\
& =\prod_{\delta \in \Delta_{1}} \prod_{i \in \Omega_{\delta}^{\sigma}} \operatorname{sgn}_{Y}(f(i)) \\
& =\prod_{i \in \Omega} \operatorname{sgn}_{Y}(f(i))
\end{aligned}
$$

by (3.2). Thus $\psi_{\Lambda_{\mathrm{sgn}}}=\operatorname{sgn}_{H}$. Let ${ }^{*}$ be the duality $\Lambda \mapsto \Lambda^{*}$, where $\Lambda^{*}: \xi_{\gamma} \mapsto$ $\left(\lambda_{p+1-\gamma}\right)^{*}$. We say $\Lambda$ is a splitting mapping of $H$ if $\psi_{\Lambda}$ is a splitting character of $H$, i.e., $\operatorname{Res}_{H^{+}}^{H}\left(\psi_{\Lambda}\right)=\psi_{\Lambda,+}+\psi_{\Lambda,-}$ is reducible on $H^{+}$. The main result of this section is an analog of Frobenius's theorem for splitting characters $\psi_{\Lambda}$.

Proposition (4D). Let $\psi_{\Lambda} \in H^{\vee}$. Then $\operatorname{sgn}_{H} \psi_{\Lambda}=\psi_{\Lambda^{*}}$. In particular, $\psi_{\Lambda}$ is a splitting character if and only if $\Lambda=\Lambda^{*}$.

Proof. Clearly

$$
\begin{aligned}
\psi_{\Lambda} \operatorname{sgn}_{H} & =\left(\operatorname{Ind}_{H_{\Lambda}}^{H}\left(\mathrm{E}\left(\xi_{\Lambda}\right) \omega_{\Lambda}\right)\right) \operatorname{sgn}_{H} \\
& =\operatorname{Ind}_{H_{\Lambda}}^{H}\left(\mathrm{E}\left(\xi_{\Lambda}\right) \omega_{\Lambda} \operatorname{sgn}_{H_{\Lambda}}\right)
\end{aligned}
$$

where $\operatorname{sgn}_{H_{\Lambda}}=\operatorname{Res}_{H_{\Lambda}}^{H}\left(\operatorname{sgn}_{H}\right)$. Let $(f, \sigma) \in H_{\Lambda}$, and write $(f, \sigma)=\prod_{\gamma}\left(f_{\gamma}, \sigma_{\gamma}\right)$, where $\left(f_{\gamma}, \sigma_{\gamma}\right)$ in $Y \imath S\left(\Omega_{\gamma}^{\Lambda}\right)$. Let $\Omega_{\gamma}^{\Lambda}=\coprod_{\delta \in \Delta_{\gamma}} \Omega_{\delta}^{\sigma}$ be the orbit decomposition of $\sigma_{\gamma}$ on $\Omega_{\gamma}^{\Lambda}$. Since

$$
\operatorname{sgn}_{H_{\Lambda}}(f, \sigma)=\prod_{\gamma}\left[\prod_{\delta \in \Delta_{\gamma}} \operatorname{sgn}_{Y}\left(p_{i_{\delta}}\left(f_{\gamma}, \sigma_{\gamma}\right)\right)\right] \operatorname{sgn}_{S\left(\Omega_{\gamma}^{\Lambda}\right)}\left(\sigma_{\gamma}\right),
$$

we have that

$$
\begin{aligned}
& \left(\mathrm{E}\left(\xi_{\Lambda}\right) \omega_{\Lambda} \operatorname{sgn}_{H_{\Lambda}}\right)(f, \sigma) \\
& \quad=\prod_{\gamma}\left[\prod_{\delta \in \Delta_{\gamma}} \xi_{\gamma}\left(p_{i_{\delta}}\left(f_{\gamma}, \sigma_{\gamma}\right)\right) \operatorname{sgn}_{Y}\left(p_{i_{\delta}}\left(f_{\gamma}, \sigma_{\gamma}\right)\right)\right] \omega_{\lambda_{\gamma}}\left(\sigma_{\gamma}\right) \operatorname{sgn}_{S\left(\Omega_{\gamma}^{\Lambda}\right)}\left(\sigma_{\gamma}\right) \\
& \quad=\prod_{\gamma}\left[\prod_{\delta \in \Delta_{\gamma}} \xi_{p+1-\gamma}\left(p_{i_{\delta}}\left(f_{\gamma}, \sigma_{\gamma}\right)\right)\right] \omega_{\lambda_{\gamma}^{*}}\left(\sigma_{\gamma}\right) \\
& \quad=\left(\mathrm{E}\left(\xi_{\Lambda^{*}}\right) \omega_{\Lambda^{*}}\right)(f, \sigma) .
\end{aligned}
$$

Thus $\psi_{\Lambda} \operatorname{sgn}_{H}=\psi_{\Lambda^{*}}$, and $\operatorname{Res}_{H^{+}}^{H}\left(\psi_{\Lambda}\right)$ is reducible if and only if $\Lambda=\Lambda^{*}$.

Each element of $H$ is $Y^{\Omega}$-conjugate to an element $(f, \sigma)$ of the following type: Let $\Omega=\coprod_{\delta}^{d} \Omega_{\delta}^{\sigma}$ be the orbit decomposition of $\sigma$, let $(f, \sigma)=\prod_{\delta}\left(f_{\delta}, \sigma_{\delta}\right)$, where $f_{\delta} \in Y^{\Omega_{\delta}^{\sigma}}$ and $\sigma_{\delta}$ is the restriction of $\sigma$ to $\Omega_{\delta}^{\sigma}$, and let $n_{\delta}=\left|\Omega_{\delta}^{\sigma}\right|$. Then $f_{\delta}$ takes a value $r_{\delta}$ in $\mathcal{R}$ on a chosen element $i_{\delta}$ in $\Omega_{\delta}^{\sigma}$ and value 1 on $\Omega_{\delta}^{\sigma}-\left\{i_{\delta}\right\}$. In particular, $p_{i_{\delta}}(f, \sigma)=r_{\delta}$. The data $\prod_{\delta}\left[r_{\delta}, n_{\delta}\right]$ is called the cycle type of $(f, \sigma)$ and characterizes the conjugacy class $\mathcal{C}$ of $(f, \sigma)$ in $H$. The cycle type of $(f, \sigma)$ in $H$ and the cycle type of $(f, \sigma)$ in $S(\Gamma \times \Omega)$ are related as follows: Let $\Gamma=\coprod_{\kappa=1}^{k_{\delta}} \Gamma_{\delta, \kappa}$ be the orbit decomposition of $\left\langle p_{i_{\delta}}(f, \sigma)\right\rangle$. The subsets $\Gamma_{\delta, \kappa} \times \Omega_{\delta}$ for $1 \leq \kappa \leq k_{\delta}$ are the orbits of $\left(f_{\delta}, \sigma_{\delta}\right)$ on $\Gamma \times \Omega_{\delta}^{\sigma}$. Thus the cycle type of $(f, \sigma)$ in $S(\Gamma \times \Omega)$ is the partition 
with parts $\left|\Gamma_{\delta, \kappa}\right|\left|\Omega_{\delta}\right|$ for $1 \leq \delta \leq d$ and $1 \leq \kappa \leq k_{\delta}$. A conjugacy class $\mathcal{C}$ of $H$ is a splitting class if $\mathcal{C} \subseteq H^{+}$and $\mathcal{C}=\mathcal{C}_{+} \cup \mathcal{C}_{-}$is the union of two conjugacy classes of $H^{+}$. The following result is $[6, \S 4.2]$.

Lemma (4E). A conjugacy class $\mathcal{C}$ of $H$ is a splitting class if and only if its cycle type satisfies the following conditions:

(i) Cycles $[a, k]$ occur only for odd $k$, and such cycles have distinct lengths $k$.

(ii) Cycles $\left[b^{i}, j\right]$ occur only for odd $i$ and even $j$.

A splitting class $\mathcal{C}$ thus has cycle type

$$
\prod_{\delta=1}^{d}\left[a, k_{\delta}\right] \prod_{i=1}^{p^{*}-1} \prod_{j}\left[b^{2 i-1}, 2 j\right]^{w_{i j}},
$$

where the $k_{\delta}$ are distinct odd integers. The cycle type of a splitting $\mathcal{C}$ is $p$-regular if no cycle of type $[a, k]$ occurs, $p$-singular if no cycle of type $\left[b^{2 i-1}, 2 j\right]$ occurs. A splitting class $\mathcal{C}$ consists of $p$-singular elements if and only if $d>0$. If $(f, \sigma) \in \mathcal{C}$, then

$$
C_{Y^{\Omega}}(f, \sigma) \simeq\langle a\rangle^{d} \times \prod_{i, j}\langle b\rangle^{w_{i j}}
$$

In particular,

$$
\left|C_{H}(f, \sigma)\right|_{p}=p^{d} .
$$

We define a bijection between splitting mappings $\Lambda$ and splitting classes $\mathcal{C}$ of $H$. If $\Lambda$ is a splitting mapping, let $\mathcal{C}_{\Lambda}$ be the splitting class of the following cycle type (4.2): $d$ is the number of diagonal nodes of $\lambda_{p^{*}}, k_{\delta}$ is the hook length $\eta_{\delta \delta}\left(\lambda_{p^{*}}\right)$, and $w_{i j}$ is the number of parts of length $j$ in $\lambda_{i}$. Conversely, if $\mathcal{C}$ is a splitting class of cycle type (4.2), let $\Lambda_{\mathcal{C}}$ be the following $p$-tuple $\left(\lambda_{1}, \lambda_{2}, \ldots, \lambda_{p}\right): \lambda_{i}$ for $i<p^{*}$ is the partition having a part $j$ for each cycle of type $\left[b^{2 i-1}, 2 j\right], \lambda_{p^{*}}$ is the unique self-dual partition such that $\eta_{\delta \delta}\left(\lambda_{p^{*}}\right)=k_{\delta}$ for $1 \leq \delta \leq d$, and $\lambda_{i}$ for $i>p^{*}$ is the partition $\lambda_{p+1-i}^{*}$. Then $\left(\Lambda_{\mathcal{C}}\right)^{*}=\Lambda_{\mathcal{C}}$ and $\Lambda_{\mathcal{C}}$ is a splitting mapping.

If $\Lambda$ is a splitting mapping of $H$, let $\Lambda^{\prime}$ and $\Lambda^{\prime \prime}$ be the $p$-tuples gotten from $\Lambda$ by replacing $\lambda_{p^{*}}$ by $\emptyset$ in the one case and by replacing $\lambda_{\gamma}$ for $\gamma \neq p^{*}$ by $\emptyset$ in the other case. We call $\Lambda^{\prime}$ and $\Lambda^{\prime \prime}$ the $p$-regular and the $p$-singular parts of $\Lambda$. The $p$-tuple with all components $\emptyset$ will be called the trivial $p$-tuple. Let $\Omega=\Omega^{\prime} \cup \Omega^{\prime \prime}$, where

$$
\Omega^{\prime}=\coprod_{\gamma \neq p^{*}} \Omega_{\gamma}^{\Lambda}, \quad \Omega^{\prime \prime}=\Omega_{p^{*}}^{\Lambda},
$$

and $\left|\Omega_{\gamma}^{\Lambda}\right|=\left|\lambda_{\gamma}\right|$ for all $\gamma$. Let $w^{\prime}=\left|\Omega^{\prime}\right|, w^{\prime \prime}=\left|\Omega^{\prime \prime}\right|, H^{\prime}=Y \imath S\left(\Omega^{\prime}\right), H^{\prime \prime}=Y \imath S\left(\Omega^{\prime \prime}\right)$. We may regard a non-trivial $\Lambda^{\prime}$ or $\Lambda^{\prime \prime}$ as a splitting mapping parametrizing a splitting character $\psi_{\Lambda^{\prime}}$ or $\psi_{\Lambda^{\prime \prime}}$ of $H^{\prime}$ or $H^{\prime \prime}$, since $\left(\Lambda^{\prime}\right)^{*}=\Lambda^{\prime}$ and $\left(\Lambda^{\prime \prime}\right)^{*}=\Lambda^{\prime \prime}$. In that case let $\left(H^{\prime}\right)^{+}=H^{\prime} \cap A\left(\Gamma \times \Omega^{\prime}\right)$ and $\left(H^{\prime \prime}\right)^{+}=H^{\prime \prime} \cap A\left(\Gamma \times \Omega^{\prime \prime}\right)$, and let

$$
\operatorname{Res}_{\left(H^{\prime}\right)^{+}}^{H^{\prime}}\left(\psi_{\Lambda^{\prime}}\right)=\psi_{\Lambda^{\prime},+}+\psi_{\Lambda^{\prime},-} \text { and } \operatorname{Res}_{\left(H^{\prime \prime}\right)^{+}}^{H^{\prime \prime}}\left(\psi_{\Lambda^{\prime \prime}}\right)=\psi_{\Lambda^{\prime \prime},+}+\psi_{\Lambda^{\prime \prime},-} .
$$

Suppose $\mathcal{C}_{\Lambda}$ has cycle type (4.2). If $\Lambda^{\prime}$ is trivial, then $H^{\prime}=\left(H^{\prime}\right)^{+}=1$ and we define $\mathcal{C}_{\Lambda^{\prime}}$ as the identity class of $H^{\prime}$. If $\Lambda^{\prime}$ is non-trivial, then $\mathcal{C}_{\Lambda^{\prime}}=\mathcal{C}_{\Lambda^{\prime},+} \cup \mathcal{C}_{\Lambda^{\prime},-}$ is a splitting class of $H^{\prime}$ of cycle type $\prod_{i, j}\left[b^{2 i-1}, 2 j\right]^{w_{i j}}$. If $\Lambda^{\prime \prime}$ is trivial, then $H^{\prime \prime}=\left(H^{\prime \prime}\right)^{+}=1$ and we define $\mathcal{C}_{\Lambda^{\prime \prime}}$ as the identity class of $H^{\prime \prime}$. If $\Lambda^{\prime \prime}$ is non-trivial, then $\mathcal{C}_{\Lambda^{\prime \prime}}=\mathcal{C}_{\Lambda^{\prime \prime},+} \cup \mathcal{C}_{\Lambda^{\prime \prime},-}$ is a splitting class of $H^{\prime \prime}$ of cycle type $\prod_{\delta}\left[a, k_{\delta}\right]$. Moreover, the irreducible character $\omega_{\lambda_{p^{*}}}$ of $S\left(\Omega^{\prime \prime}\right)$ splits as $\omega_{\lambda_{p^{*},+}}+\omega_{\lambda_{p^{*},-}}$ on $A\left(\Omega^{\prime \prime}\right)$. 
Proposition (4F). Suppose $\Lambda$ is a splitting mapping of $H$ such that $\Lambda$ equals its $p$-singular part $\Lambda^{\prime \prime}$. Let $(f, \sigma) \in H^{+}$. Then $\left(\psi_{\Lambda,+}-\psi_{\Lambda,-}\right)(f, \sigma) \neq 0$ if and only if $(f, \sigma) \in \mathcal{C}_{\Lambda}$. Moreover, $\psi_{\Lambda, \pm}$ and $\mathcal{C}_{\Lambda, \pm}$ may be labeled so that

$$
\left(\psi_{\Lambda,+}-\psi_{\Lambda,-}\right)(f, \sigma)= \pm\left(\sqrt{\epsilon_{p} p}\right)^{d} \sqrt{\epsilon_{\lambda_{p^{*}}} \eta\left(\lambda_{p^{*}}\right)} \quad \text { for }(f, \sigma) \in \mathcal{C}_{\Lambda, \pm},
$$

where $d$ is the number of diagonal nodes in $\lambda_{p^{*}}$.

Proof. For simplicity of notation we write $\xi$ for $\xi_{p^{*}}$ and $\lambda$ for $\lambda_{p^{*}}$. By hypothesis $H_{\Lambda}=H$ and $\psi_{\Lambda}=\mathrm{E}\left(\xi_{\Lambda}\right) \omega_{\Lambda}$. Suppose $w=1$. Then $H=Y, H^{+}=Y^{+}, \psi_{\Lambda}=\xi$, and $\mathcal{C}_{\Lambda}=a^{Y}$. So $(4 \mathrm{~F})$ holds if we choose $\psi_{\Lambda, \pm}=\xi_{ \pm}$and $\mathcal{C}_{\Lambda, \pm}=\left(a_{ \pm}\right)^{Y^{+}}$. We may suppose $w>1$. The irreducible character $\theta_{\Lambda}=\left(\xi_{+}\right)^{\Omega}$ of $\left(Y^{+}\right)^{\Omega}$ is stable under $Y^{+} \imath S(\Omega)$. Let $\mathrm{E}\left(\theta_{\Lambda}\right)$ be its James-Kerber extension to $Y^{+} \imath S(\Omega)$. Since $\xi=\operatorname{Ind}_{Y^{+}}^{Y}\left(\xi_{+}\right)$, it follows by $(4 \mathrm{C})$ that

$$
\mathrm{E}\left(\xi_{\Lambda}\right)=\operatorname{Ind}_{Y+\imath S(\Omega)}^{H}\left(\mathrm{E}\left(\theta_{\Lambda}\right)\right) .
$$

Frobenius reciprocity and Mackey decomposition give

$$
\begin{aligned}
\operatorname{Res}_{H^{+}}^{H}\left(\psi_{\Lambda}\right) & =\operatorname{Res}_{H^{+}}^{H}\left(\mathrm{E}\left(\xi_{\Lambda}\right) \omega_{\Lambda}\right) \\
& =\operatorname{Res}_{H^{+}}^{H}\left(\operatorname{Ind}_{Y^{+} \imath S(\Omega)}^{H}\left(\mathrm{E}\left(\theta_{\Lambda}\right)\right)\right) \operatorname{Res}_{H^{+}}^{H}\left(\omega_{\Lambda}\right) \\
& =\operatorname{Ind}_{Y^{+} \imath A(\Omega)}^{H^{+}}\left(\operatorname{Res}_{Y^{+} \imath A(\Omega)}^{Y^{+}(\Omega)}\left(\mathrm{E}\left(\theta_{\Lambda}\right)\right)\right) \operatorname{Res}_{H^{+}}^{H}\left(\omega_{\Lambda}\right) .
\end{aligned}
$$

Let $\left(Y^{\Omega}\right)^{+}=Y^{\Omega} \cap G^{+}$and let $\Theta_{\Lambda,+}=\operatorname{Ind}_{Y^{+} \imath A(\Omega)}^{\left(Y^{\Omega}\right)^{+} A(\Omega)}\left(\operatorname{Res}_{Y^{+}(A)(\Omega)}^{Y^{+}(\Omega(\Omega)}\left(\mathrm{E}\left(\theta_{\Lambda}\right)\right)\right)$. Then

$$
\begin{aligned}
\operatorname{Res}_{H^{+}}^{H}\left(\psi_{\Lambda}\right) & =\operatorname{Ind}_{\left(Y^{\Omega}\right)^{+} A(\Omega)}^{H^{+}}\left(\Theta_{\Lambda,+}\right) \operatorname{Res}_{H^{+}}^{H}\left(\omega_{\Lambda}\right) \\
& =\operatorname{Ind}_{\left(Y^{\Omega}\right)^{+} A(\Omega)}^{H^{+}}\left(\Theta_{\Lambda,+} \operatorname{Res}_{\left(Y^{\Omega}\right)^{+} A(\Omega)}^{H}\left(\omega_{\Lambda}\right)\right) \\
& =\operatorname{Ind}_{\left(Y^{\Omega}\right)^{+} A(\Omega)}^{H^{+}}\left(\Theta_{\Lambda,+}\left(\omega_{\Lambda,+}+\omega_{\Lambda,-}\right)\right) .
\end{aligned}
$$

Since $\operatorname{Res}_{H^{+}}^{H}\left(\psi_{\Lambda}\right)=\psi_{\Lambda,+}+\psi_{\Lambda,-}$, it follows that $\Theta_{\Lambda,+}$ is an irreducible character of $\left(Y^{\Omega}\right)^{+} A(\Omega)$. We label $\psi_{\Lambda, \pm}$ so that

$$
\begin{aligned}
& \psi_{\Lambda,+}=\operatorname{Ind}_{\left(Y^{\Omega}\right)^{+} A(\Omega)}^{H^{+}}\left(\Theta_{\Lambda,+} \omega_{\Lambda,+}\right), \\
& \psi_{\Lambda,-}=\operatorname{Ind}_{\left(Y^{\Omega}\right)^{+} A(\Omega)}^{H^{+}}\left(\Theta_{\Lambda,+} \omega_{\Lambda,-}\right) .
\end{aligned}
$$

Let $H^{+}=\left\langle\left(Y^{\Omega}\right)^{+} A(\Omega), s\right\rangle$, where $s=(g, \tau)$ with $g \notin\left(Y^{\Omega}\right)^{+}$and $\tau \notin A(\Omega)$, and set $\Theta_{\Lambda,-}=\left(\Theta_{\Lambda,+}\right)^{s}$. Then

$$
\begin{aligned}
& \operatorname{Res}_{\left(Y^{+}\right)^{\Omega}}^{\left(Y^{\Omega}\right)}\left(\Theta_{\Lambda,+}\right)=\sum_{\substack{\left(\epsilon_{1}, \epsilon_{2}, \ldots, \epsilon_{w}\right) \\
\epsilon_{1} \epsilon_{2} \cdots \epsilon_{w}=+}} \xi_{\epsilon_{1}} \times \xi_{\epsilon_{2}} \times \cdots \times \xi_{\epsilon_{w}}, \\
& \operatorname{Res}_{\left(Y^{+}\right)^{\Omega}}^{\left(Y^{\Omega}\right)^{+} A(\Omega)}\left(\Theta_{\Lambda,-}\right)=\sum_{\substack{\left(\epsilon_{1}, \epsilon_{2}, \ldots, \epsilon_{w}\right) \\
\epsilon_{1} \epsilon_{2} \cdots \epsilon_{w}=-}} \xi_{\epsilon_{1}} \times \xi_{\epsilon_{2}} \times \cdots \times \xi_{\epsilon_{w}} .
\end{aligned}
$$

In particular, $\Theta_{\Lambda,-} \neq \Theta_{\Lambda,+}$. By $(4.5)$

$$
\psi_{\Lambda,+}-\psi_{\Lambda,-}= \begin{cases}\left(\Theta_{\Lambda,+}-\Theta_{\Lambda,-}\right)\left(\omega_{\Lambda,+}-\omega_{\Lambda,-}\right) & \text { on }\left(Y^{\Omega}\right)^{+} A(\Omega), \\ 0 & \text { off }\left(Y^{\Omega}\right)^{+} A(\Omega) .\end{cases}
$$


Suppose $\left(\psi_{\Lambda,+}-\psi_{\Lambda,-}\right)(f, \sigma) \neq 0$. Then $(f, \sigma) \in\left(Y^{\Omega}\right)^{+} A(\Omega)$ by (4.6), and

$$
\left(\Theta_{\Lambda,+}-\Theta_{\Lambda,-}\right)(f, \sigma) \neq 0, \quad\left(\omega_{\Lambda,+}-\omega_{\Lambda,-}\right)(\sigma) \neq 0 .
$$

(4A) implies that $\sigma$ is in the class $\mathcal{C}_{\eta(\lambda)}$ of $S(\Omega)$ and

$$
\left(\omega_{\Lambda,+}-\omega_{\Lambda,-}\right)(\sigma)= \pm \sqrt{\epsilon_{\lambda} \eta(\lambda)} .
$$

Since $(f, \sigma)$ is in a splitting class of $H,(4 \mathrm{E})$ then implies that $(f, \sigma) \in \mathcal{C}_{\Lambda}$. We may suppose $p_{i_{\delta}}((f, \sigma))=a$ for all $\delta$. The proof of $(4 \mathrm{C})$ then gives

$$
\Theta_{\Lambda,+}((f, \sigma))=\sum_{h \in\left(Y^{\Omega}\right)^{+} /\left(Y^{+}\right)^{\Omega}} \mathrm{E}\left(\theta_{\Lambda}\right)\left({ }^{h}(f, \sigma)\right)=\sum_{\substack{\left(\epsilon_{1}, \epsilon_{2}, \ldots, \epsilon_{d}\right) \\ \epsilon_{1} \epsilon_{2} \cdots \epsilon_{d}=+}} \prod_{\delta=1}^{d} \xi_{\epsilon_{\delta}}(a) .
$$

Since $\Theta_{\Lambda,+}$ is $\left(Y^{\Omega}\right)^{+} S(\Omega)$-invariant and $\Theta_{\Lambda,-}=\left(\Theta_{\Lambda,+}\right)^{s}$,

$$
\Theta_{\Lambda,-}((f, \sigma))=\sum_{\substack{\left(\epsilon_{1}, \epsilon_{2}, \ldots, \epsilon_{d}\right) \\ \epsilon_{1} \epsilon_{2} \ldots \epsilon_{d}=-}} \prod_{\delta=1}^{d} \xi_{\epsilon_{\delta}}(a)
$$

Thus

$$
\left(\Theta_{\Lambda,+}-\Theta_{\Lambda,-}\right)((f, \sigma))=\left(\xi_{+}(a)-\xi_{-}(a)\right)^{d}=\left(\sqrt{\epsilon_{p} p}\right)^{d} .
$$

We then label $\psi_{\Lambda, \pm}$ and $\mathcal{C}_{\Lambda, \pm}$ so that (4F) holds.

Proposition (4G). Suppose $\Lambda$ is a splitting mapping of $H$ such that $\Lambda$ equals its $p$-regular part $\Lambda^{\prime}$. Let $(f, \sigma) \in H^{+}$. Then $\left(\psi_{\Lambda,+}-\psi_{\Lambda,-}\right)(f, \sigma) \neq 0$ only if $(f, \sigma)$ is in a p-regular splitting class of $H$.

Proof. Let $H_{\Lambda}^{+}=H_{\Lambda} \cap H^{+}$and $\Theta_{\Lambda}=\operatorname{Res}_{H_{\Lambda}^{+}}^{H_{\Lambda}}\left(\mathrm{E}\left(\xi_{\Lambda}\right) \omega_{\Lambda}\right)$. Then $\Theta_{\Lambda}$ is irreducible since $\left(\mathrm{E}\left(\xi_{\Lambda}\right) \omega_{\Lambda}\right)(h, 1) \neq 0$ for any element $h$ in $Y^{\Omega}-\left(Y^{\Omega}\right)^{+}$taking value $b$ on some $i \in \Omega$ and value 1 on $\Omega-\{i\}$. We fix such an $h$. Let $\tau$ be an involution in $S(\Omega)$ interchanging $\Omega_{\gamma}^{\Lambda}$ and $\Omega_{p+1-\gamma}^{\Lambda}$ for $1 \leq \gamma<p^{*}$. Let $t=(g, \tau)$, where $g=1$ if $\tau \in A(\Omega)$ and $g=h$ if $\tau \notin A(\Omega)$. Then $t \in N_{H^{+}}\left(H_{\Lambda}^{+}\right)$and $T_{\Lambda}=\left\langle H_{\Lambda}^{+}, t\right\rangle$ is an extension of $H_{\Lambda}^{+}$of order 2 .

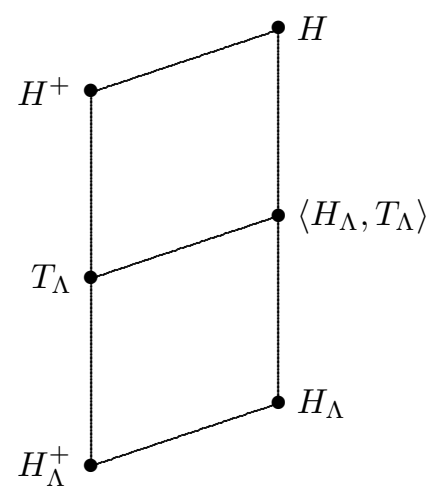


We claim $T_{\Lambda}$ stabilizes $\Theta_{\Lambda}$. Indeed, let $(f, \sigma) \in H_{\Lambda}^{+}$, and write $(f, \sigma)=\prod_{\gamma}^{p}\left(f_{\gamma}, \sigma_{\gamma}\right)$, where $f_{\gamma} \in Y^{\Omega_{\gamma}^{\Lambda}}, \sigma_{\gamma} \in S\left(\Omega_{\gamma}^{\Lambda}\right)$. Then

$$
\begin{aligned}
\omega_{\Lambda}^{t}(\sigma) & =\prod_{\gamma=1}^{p} \omega_{\lambda_{\gamma}}\left({ }^{t} \sigma_{\gamma}\right) \\
& =\prod_{\gamma=1}^{p} \omega_{\lambda_{\gamma}^{*}}\left(\sigma_{\gamma}\right) \\
& =\prod_{\gamma=1}^{p} \omega_{\lambda_{\gamma}}\left(\sigma_{\gamma}\right) \operatorname{sgn}_{S\left(\Omega_{\gamma}^{\Lambda}\right)}\left(\sigma_{\gamma}\right) \\
& =\left(\omega_{\Lambda} \operatorname{sgn}_{S(\Omega)}\right)(\sigma) .
\end{aligned}
$$

On the other hand, if $\left(f_{\gamma}, \sigma_{\gamma}\right)$ decomposes into cycles $\prod_{\delta \in \Delta_{\gamma}}\left(f_{\delta}, \sigma_{\delta}\right)$, then

$$
\begin{aligned}
\mathrm{E}\left(\xi_{\Lambda}\right)^{t}(f, \sigma) & =\mathrm{E}\left(\xi_{\Lambda}\right)^{(g, 1)}\left({ }^{(1, \tau)}(f, \sigma)\right) \\
& =\mathrm{E}\left(\xi_{\Lambda}\right)\left({ }^{(1, \tau)}(f, \sigma)\right) \\
& =\prod_{\gamma} \prod_{\delta \in \Delta_{\gamma}} \xi_{p+1-\gamma}\left(p_{\tau\left(i_{\delta}\right)}\left({ }^{(1, \tau)}(f, \sigma)\right)\right) \\
& =\prod_{\gamma} \prod_{\delta \in \Delta_{\gamma}} \xi_{\gamma}^{*}\left(p_{i_{\delta}}(f, \sigma)\right) \\
& =\prod_{\gamma} \prod_{\delta \in \Delta_{\gamma}}\left(\xi_{\gamma}\left(p_{i_{\delta}}(f, \sigma)\right) \operatorname{sgn}_{Y}\left(p_{i_{\delta}}(f, \sigma)\right)\right) \\
& =\mathrm{E}\left(\xi_{\Lambda}\right)(f, \sigma) \operatorname{sgn}_{S(\Omega)}(\sigma),
\end{aligned}
$$

since $\prod_{\gamma} \prod_{i_{\delta} \in \Delta_{\gamma}} \operatorname{sgn}_{Y}\left(p_{i_{\delta}}(f, \sigma)=\prod_{i \in \Omega} \operatorname{sgn}_{Y}(f(i))=\operatorname{sgn}_{S(\Omega)}(\sigma)\right.$. So $T_{\Lambda}$ stabilizes $\Theta_{\Lambda}$ by (4.7) and (4.8).

Let $\Theta_{\Lambda}^{+}$and $\Theta_{\Lambda}^{-}$be the two extensions of $\Theta_{\Lambda}$ to $T_{\Lambda}$. By Mackey decomposition we may label $\psi_{\Lambda, \pm}$ so that

$$
\psi_{\Lambda,+}=\operatorname{Ind}_{T_{\Lambda}}^{H^{+}}\left(\Theta_{\Lambda}^{+}\right), \quad \psi_{\Lambda,-}=\operatorname{Ind}_{T_{\Lambda}}^{H^{+}}\left(\Theta_{\Lambda}^{-}\right) .
$$

We note for later use that any odd element $s$ of $Y^{\Omega}$ normalizes $T_{\Lambda}$ and interchanges $\Theta_{\Lambda}^{+}$and $\Theta_{\Lambda}^{-}$, since $\psi_{\Lambda,+}^{s}=\psi_{\Lambda,-}$.

Suppose $\left(\psi_{\Lambda,+}-\psi_{\Lambda,-}\right)(f, \sigma) \neq 0$. Replacing $(f, \sigma)$ by a conjugate, we may suppose by $(4.9)$ that $(f, \sigma) \in T_{\Lambda}$ and $\left(\Theta_{\Lambda}^{+}-\Theta_{\Lambda}^{-}\right)(f, \sigma) \neq 0$. Thus $(f, \sigma) \notin H_{\Lambda}^{+}$. Elements of $T_{\Lambda}-H_{\Lambda}^{+}$have the form $(h, \rho)$, where $\rho$ interchanges $\coprod_{\gamma<p^{*}} \Omega_{\gamma}^{\Lambda}$ and $\bigsqcup_{\gamma>p^{*}} \Omega_{\gamma}^{\Lambda}$. In particular, $\rho$ contains no cycles of odd length. Thus $(f, \sigma)$ is contained in a $p$-regular splitting class of $H$. This completes the proof of $(4 \mathrm{G})$.

Proposition (4H). Suppose $\Lambda$ is a splitting mapping of $H, \Lambda^{\prime}$ and $\Lambda^{\prime \prime}$ are the $p$ regular and p-singular parts of $\Lambda$, and $H^{\prime}$ and $H^{\prime \prime}$ are the subgroups of $H$ associated to $\Lambda^{\prime}$ and $\Lambda^{\prime \prime}$.

(i) If $\left(\psi_{\Lambda,+}-\psi_{\Lambda,-}\right)(f, \sigma) \neq 0$, then $(f, \sigma)$ is $H^{+}$-conjugate to $\left(f^{\prime}, \sigma^{\prime}\right)\left(f^{\prime \prime}, \sigma^{\prime \prime}\right)$, where $\left(f^{\prime}, \sigma^{\prime}\right)$ is in a p-regular splitting class of $H^{\prime}$ and $\left(f^{\prime \prime}, \sigma^{\prime \prime}\right)$ is in the p-singular splitting class $\mathcal{C}_{\Lambda^{\prime \prime}}$ of $H^{\prime \prime}$. 
(ii) Given labelings of $\psi_{\Lambda^{\prime}, \pm}$ and $\psi_{\Lambda^{\prime \prime}, \pm}$, there exists a labeling of $\psi_{\Lambda, \pm}$ such that

$$
\begin{aligned}
\left(\psi_{\Lambda,+}-\psi_{\Lambda,-}\right)(f, \sigma)= & \left(\psi_{\Lambda^{\prime},+}-\psi_{\Lambda^{\prime},-}\right)\left(f^{\prime}, \sigma^{\prime}\right) \\
& \times\left(\psi_{\Lambda^{\prime \prime},+}-\psi_{\Lambda^{\prime \prime},-}\right)\left(f^{\prime \prime}, \sigma^{\prime \prime}\right)
\end{aligned}
$$

for all $(f, \sigma)=\left(f^{\prime}, \sigma^{\prime}\right)\left(f^{\prime \prime}, \sigma^{\prime \prime}\right)$ with $\left(f^{\prime}, \sigma^{\prime}\right)$ a p-regular element of $\left(H^{\prime}\right)^{+}$ and $\left(f^{\prime \prime}, \sigma^{\prime \prime}\right)$ an arbitrary element of $\left(H^{\prime \prime}\right)^{+}$.

Proof. $(4 \mathrm{H})$ follows trivially from $(4 \mathrm{~F})$ or $(4 \mathrm{G})$ if $\Lambda^{\prime}$ or $\Lambda^{\prime \prime}$ is trivial. So we may suppose $\Lambda^{\prime}$ and $\Lambda^{\prime \prime}$ are non-trivial Let $\xi_{\Lambda}$ be the irreducible character $\prod_{\gamma}\left(\xi_{\gamma}\right)^{\Omega_{\gamma}^{\Lambda}}$ of $Y^{\Omega}$. Let $\xi_{\Lambda^{\prime}}$ and $\xi_{\Lambda^{\prime \prime}}$ be the irreducible characters of $Y^{\Omega^{\prime}}$ and $Y^{\Omega^{\prime \prime}}$ defined by

$$
\xi_{\Lambda^{\prime}}=\prod_{\gamma \neq p^{*}}\left(\xi_{\gamma}\right)^{\Omega_{\gamma}^{\Lambda}}, \quad \xi_{\Lambda^{\prime \prime}}=\left(\xi_{p^{*}}\right)^{\Omega_{p^{*}}^{\Lambda}}
$$

The stabilizers of $\xi_{\Lambda^{\prime}}$ in $H^{\prime}$ and $\xi_{\Lambda^{\prime \prime}}$ in $H^{\prime \prime}$ are $H_{\Lambda^{\prime}}=Y^{\Omega^{\prime}} S\left(\Omega^{\Lambda^{\prime}}\right)$ and $H_{\Lambda^{\prime \prime}}=$ $Y^{\Omega^{\prime \prime}} S\left(\Omega^{\Lambda^{\prime \prime}}\right)$, where $S\left(\Omega^{\Lambda^{\prime}}\right)$ and $S\left(\Omega^{\Lambda^{\prime \prime}}\right)$ are the components of $S\left(\Omega^{\Lambda}\right)$ on $\Omega^{\prime}$ and $\Omega^{\prime \prime}$. Let $\mathrm{E}\left(\xi_{\Lambda^{\prime}}\right)$ and $\mathrm{E}\left(\xi_{\Lambda^{\prime \prime}}\right)$ be the James-Kerber extensions of $\xi_{\Lambda^{\prime}}$ and $\xi_{\Lambda^{\prime \prime}}$ to $H_{\Lambda^{\prime}}$ and $H_{\Lambda^{\prime \prime}}$, and let $\omega_{\Lambda^{\prime}}$ and $\omega_{\Lambda^{\prime \prime}}$ be the irreducible characters of $S\left(\Omega^{\Lambda^{\prime}}\right)$ and $S\left(\Omega^{\Lambda^{\prime \prime}}\right)$ parametrized by $\Lambda^{\prime}$ and $\Lambda^{\prime \prime}$. Then

$$
\psi_{\Lambda^{\prime}}=\operatorname{Ind}_{H_{\Lambda^{\prime}}}^{H^{\prime}}\left(\mathrm{E}\left(\xi_{\Lambda^{\prime}}\right) \omega_{\Lambda^{\prime}}\right), \quad \psi_{\Lambda^{\prime \prime}}=\mathrm{E}\left(\xi_{\Lambda^{\prime \prime}}\right) \omega_{\Lambda^{\prime \prime}},
$$

and

$$
\mathrm{E}\left(\xi_{\Lambda}\right) \omega_{\Lambda}=\mathrm{E}\left(\xi_{\Lambda^{\prime}}\right) \omega_{\Lambda^{\prime}} \times \mathrm{E}\left(\xi_{\Lambda^{\prime \prime}}\right) \omega_{\Lambda^{\prime \prime}}
$$

Let

$$
\Theta_{\Lambda}=\operatorname{Res}_{H_{\Lambda}^{+}}^{H_{\Lambda}}\left(\mathrm{E}\left(\xi_{\Lambda}\right) \omega_{\Lambda}\right), \quad \Theta_{\Lambda^{\prime}}=\operatorname{Res}_{H_{\Lambda^{\prime}}^{+}}^{H_{\Lambda^{\prime}}}\left(\mathrm{E}\left(\xi_{\Lambda^{\prime}}\right) \omega_{\Lambda^{\prime}}\right)
$$

Then $\Theta_{\Lambda}$ and $\Theta_{\Lambda^{\prime}}$ are irreducible since $\left(\mathrm{E}\left(\xi_{\Lambda}\right) \omega_{\Lambda}\right)\left(f^{\prime}, 1\right)$ and $\left(\mathrm{E}\left(\xi_{\Lambda^{\prime}}\right) \omega_{\Lambda^{\prime}}\right)\left(f^{\prime}, 1\right)$ are non-zero for any element $f^{\prime}$ in $Y^{\Omega^{\prime}}-\left(Y^{\Omega^{\prime}}\right)^{+}$taking value $b$ on some $i \in \Omega^{\prime}$ and value 1 on $\Omega^{\prime}-\{i\}$. We fix such an $f^{\prime}$. On the other hand,

$$
\operatorname{Res}_{\left(H^{\prime \prime}\right)+}^{H^{\prime \prime}}\left(\psi_{\Lambda^{\prime \prime}}\right)=\psi_{\Lambda^{\prime \prime},+}+\psi_{\Lambda^{\prime \prime},-}, \quad \operatorname{Res}_{H_{\Lambda^{\prime}}^{+} \times H_{\Lambda^{\prime \prime}}^{+}}^{H_{+}^{+}}\left(\Theta_{\Lambda}\right)=\Theta_{\Lambda,+}+\Theta_{\Lambda,-}
$$

are reducible. Given a labeling of $\psi_{\Lambda^{\prime \prime}, \pm}$, we label $\Theta_{\Lambda, \pm}$ so that $\Theta_{\Lambda,+}=\Theta_{\Lambda^{\prime}} \times \psi_{\Lambda^{\prime \prime},+}$ and $\Theta_{\Lambda,-}=\Theta_{\Lambda^{\prime}} \times \psi_{\Lambda^{\prime \prime},-}$.

Let $s=(h, 1)$ in $Y^{\Omega}$ be such that $h$ takes the value $b$ once on $\Omega^{\prime}$ and once on $\Omega^{\prime \prime}$, and the value 1 everywhere else. Then $H_{\Lambda}^{+}=\left\langle H_{\Lambda^{\prime}}^{+} \times H_{\Lambda^{\prime \prime}}^{+}, s\right\rangle$. Let $\tau \in S\left(\Omega^{\prime}\right)$ be an involution interchanging $\Omega_{\gamma}^{\Lambda}$ and $\Omega_{p+1-\gamma}^{\Lambda}$ for $1 \leq \gamma<p^{*}$. Let $t=(g, \tau)$, where $g$ is the identity of $Y^{\Omega^{\prime}}$ if $\tau \in A\left(\Omega^{\prime}\right)$, and $g=f^{\prime}$ if $\tau \notin A\left(\Omega^{\prime}\right)$. Then $t \in N_{\left(H^{\prime}\right)}\left(H_{\Lambda^{\prime}}^{+}\right)$, $T_{\Lambda^{\prime}}=\left\langle H_{\Lambda^{\prime}}^{+}, t\right\rangle$ is an extension of $H_{\Lambda^{\prime}}^{+}$of order 2 , and $[s, t] \in H_{\Lambda^{\prime}}^{+} \times H_{\Lambda^{\prime \prime}}^{+}$. In 
particular, if $T_{\Lambda}=\left\langle H_{\Lambda}^{+}, t\right\rangle$, then $T_{\Lambda}=\left\langle H_{\Lambda^{\prime}}^{+} \times H_{\Lambda^{\prime \prime}}^{+}, s, t\right\rangle=\left\langle T_{\Lambda^{\prime}} \times H_{\Lambda^{\prime \prime}}^{+}, s\right\rangle$.

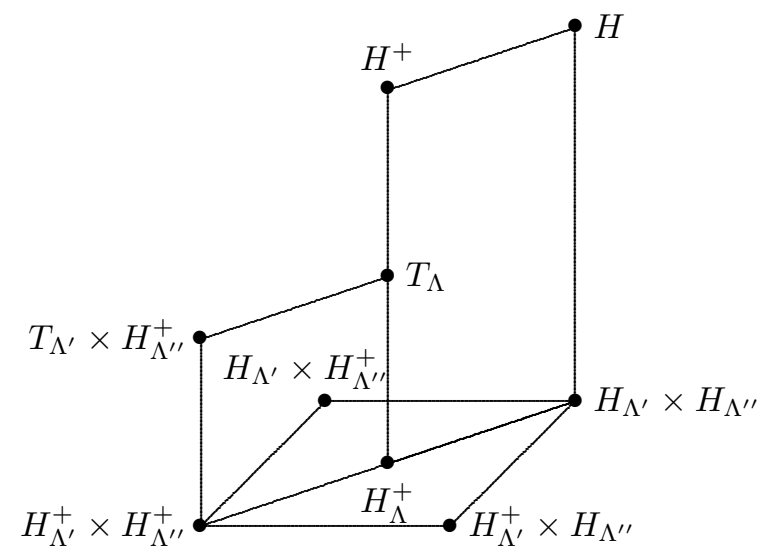

$T_{\Lambda^{\prime}}$ stabilizes $\Theta_{\Lambda^{\prime}}$ as in the proof of $(4 \mathrm{G})$. In particular, $\Theta_{\Lambda^{\prime}}$ has two extensions $\Theta_{\Lambda^{\prime}}^{+}$ and $\Theta_{\Lambda^{\prime}}^{-}$to $T_{\Lambda^{\prime}}, \operatorname{Ind}_{H_{\Lambda^{\prime}}^{+}}^{T_{\Lambda^{\prime}}}\left(\Theta_{\Lambda^{\prime}}\right)=\Theta_{\Lambda^{\prime}}^{+}+\Theta_{\Lambda^{\prime}}^{-}$, and $\operatorname{Ind}_{T_{\Lambda^{\prime}}}^{\left(H^{\prime}\right)^{+}}\left(\Theta_{\Lambda^{\prime}}^{+}+\Theta_{\Lambda^{\prime}}^{-}\right)=\psi_{\Lambda^{\prime},+}+\psi_{\Lambda^{\prime},-}$. Given a labeling of $\psi_{\Lambda^{\prime}, \pm}$, we label $\Theta_{\Lambda^{\prime}}^{ \pm}$so that

$$
\psi_{\Lambda^{\prime},+}=\operatorname{Ind}_{T_{\Lambda^{\prime}}}^{\left(H^{\prime}\right)^{+}}\left(\Theta_{\Lambda^{\prime}}^{+}\right), \quad \psi_{\Lambda^{\prime},-}=\operatorname{Ind}_{T_{\Lambda^{\prime}}}^{\left(H^{\prime}\right)^{+}}\left(\Theta_{\Lambda^{\prime}}^{-}\right) .
$$

Then $T_{\Lambda^{\prime}} \times H_{\Lambda^{\prime \prime}}^{+}$stabilizes $\Theta_{\Lambda}$ since $\Theta_{\Lambda}=\operatorname{Ind}_{H_{\Lambda^{\prime}}^{+} \times H_{\Lambda^{\prime \prime}}^{+}}^{H_{+}^{+}}\left(\Theta_{\Lambda,+}\right)$, and $T_{\Lambda}$ stabilizes $\Theta_{\Lambda}$ since $s \in H_{\Lambda}^{+}$. Now

$$
\begin{aligned}
& \left(\Theta_{\Lambda^{\prime}}^{+} \times \psi_{\Lambda^{\prime \prime},+}\right)^{s}=\Theta_{\Lambda^{\prime}}^{-} \times \psi_{\Lambda^{\prime \prime},-}, \\
& \left(\Theta_{\Lambda^{\prime}}^{+} \times \psi_{\Lambda^{\prime \prime},-}\right)^{s}=\Theta_{\Lambda^{\prime}}^{-} \times \psi_{\Lambda^{\prime \prime},+},
\end{aligned}
$$

since any odd element of $Y^{\Omega^{\prime}}$ normalizes $T_{\Lambda^{\prime}}$ and interchanges $\Theta_{\Lambda^{\prime}}^{+}$and $\Theta_{\Lambda^{\prime}}^{-}$, and any odd element of $Y^{\Omega^{\prime \prime}}$ interchanges $\psi_{\Lambda^{\prime \prime},+}$ and $\psi_{\Lambda^{\prime \prime},-}$. Thus

$$
\begin{aligned}
& \Xi_{\Lambda,+}=\operatorname{Ind}_{T_{\Lambda^{\prime} \times H_{\Lambda^{\prime \prime}}^{+}}}^{T_{\Lambda}}\left(\Theta_{\Lambda^{\prime}}^{+} \times \psi_{\Lambda^{\prime \prime},+}\right), \\
& \Xi_{\Lambda,-}=\operatorname{Ind}_{T_{\Lambda^{\prime}} \times H_{\Lambda^{\prime \prime}}^{+}}^{T_{\Lambda}}\left(\Theta_{\Lambda^{\prime}}^{+} \times \psi_{\Lambda^{\prime \prime},-}\right)
\end{aligned}
$$

are distinct irreducible characters, and

$$
\Xi_{\Lambda,+}-\Xi_{\Lambda,-}= \begin{cases}\left(\Theta_{\Lambda^{\prime}}^{+}-\Theta_{\Lambda^{\prime}}^{-}\right) \times\left(\psi_{\Lambda^{\prime \prime},+}-\psi_{\Lambda^{\prime \prime},-}\right) & \text { on } T_{\Lambda^{\prime}} \times H_{\Lambda^{\prime \prime}}^{+} \\ 0 & \text { on } T_{\Lambda}-\left(T_{\Lambda^{\prime}} \times H_{\Lambda^{\prime \prime}}^{+}\right)\end{cases}
$$

We may label $\psi_{\Lambda, \pm}$ so that

$$
\begin{aligned}
& \psi_{\Lambda,+}=\operatorname{Ind}_{T_{\Lambda}^{+}}^{H^{+}}\left(\Xi_{\Lambda,+}\right)=\operatorname{Ind}_{T_{\Lambda^{\prime}} \times H_{\Lambda^{\prime \prime}}^{+}}^{H^{+}}\left(\Theta_{\Lambda^{\prime}}^{+} \times \psi_{\Lambda^{\prime \prime},+}\right), \\
& \psi_{\Lambda,-}=\operatorname{Ind}_{T_{\Lambda}}^{H^{+}}\left(\Xi_{\Lambda,-}\right)=\operatorname{Ind}_{T_{\Lambda^{\prime}} \times H_{\Lambda^{\prime \prime}}^{+}}^{H^{+}}\left(\Theta_{\Lambda^{\prime}}^{+} \times \psi_{\Lambda^{\prime \prime},-}\right) .
\end{aligned}
$$

Indeed, Mackey decomposition implies that

$$
\operatorname{Res}_{H^{+}}^{H}\left(\psi_{\Lambda}\right)=\operatorname{Ind}_{H_{\Lambda}^{+}}^{H^{+}}\left(\Theta_{\Lambda}\right)=\operatorname{Ind}_{T_{\Lambda}}^{H^{+}}\left(\operatorname{Ind}_{H_{\Lambda}^{+}}^{T_{\Lambda}}\left(\Theta_{\Lambda}\right)\right),
$$


since $\psi_{\Lambda}=\operatorname{Ind}_{H_{\Lambda}}^{H}\left(\mathrm{E}\left(\xi_{\Lambda}\right) \omega_{\Lambda}\right)$. But

$$
\begin{aligned}
\operatorname{Ind}_{H_{\Lambda}^{+}}^{T_{\Lambda}}\left(\Theta_{\Lambda}\right) & =\operatorname{Ind}_{H_{\Lambda}^{+}}^{T_{\Lambda}}\left(\operatorname{Ind}_{H_{\Lambda^{\prime}}^{+} \times H_{\Lambda^{\prime \prime}}^{+}}^{H_{+}^{+}}\left(\Theta_{\Lambda^{\prime}} \times \psi_{\Lambda^{\prime \prime},+}\right)\right) \\
& =\operatorname{Ind}_{T_{\Lambda} \times H_{\Lambda^{\prime \prime}}^{+}}^{T_{\Lambda}}\left(\operatorname{Ind}_{H_{\Lambda^{\prime}}^{+} \times H_{\Lambda^{\prime \prime}}^{+}}^{T_{\Lambda^{\prime \prime}}^{+}}\left(\Theta_{\Lambda^{\prime}} \times \psi_{\Lambda^{\prime \prime},+}\right)\right) \\
& =\operatorname{Ind}_{T_{\Lambda^{\prime}} \times H_{\Lambda^{\prime \prime}}^{+}}^{T_{\Lambda}}\left(\left(\Theta_{\Lambda^{\prime}}^{+}+\Theta_{\Lambda^{\prime}}^{-}\right) \times \psi_{\Lambda^{\prime \prime},+}\right) \\
& =\Xi_{\Lambda,+}+\Xi_{\Lambda,-} .
\end{aligned}
$$

Thus (4.12) holds since $\operatorname{Res}_{H^{+}}^{H}\left(\psi_{\Lambda}\right)=\psi_{\Lambda,+}+\psi_{\Lambda,-}$.

Suppose $\left(\psi_{\Lambda,+}-\psi_{\Lambda,-}\right)(f, \sigma) \neq 0$. By $(4.11)$ and (4.12) there exist $\left(f^{\prime}, \sigma^{\prime}\right)$ in $T_{\Lambda^{\prime}}-H_{\Lambda^{\prime}}^{+}$and $\left(f^{\prime \prime}, \sigma^{\prime \prime}\right)$ in $H_{\Lambda^{\prime \prime}}^{+}$such that $(f, \sigma)$ is $H^{+}$-conjugate to $\left(f^{\prime}, \sigma^{\prime}\right)\left(f^{\prime \prime}, \sigma^{\prime \prime}\right)$ and

$$
\left(\Theta_{\Lambda^{\prime}}^{+}-\Theta_{\Lambda^{\prime}}^{-}\right)\left(f^{\prime}, \sigma^{\prime}\right) \neq 0, \quad\left(\psi_{\Lambda^{\prime \prime},+}-\psi_{\Lambda^{\prime \prime},-}\right)\left(f^{\prime \prime}, \sigma^{\prime \prime}\right) \neq 0
$$

$(4 \mathrm{H}),(\mathrm{i})$ now follows by $(4 \mathrm{~F})$ and the last paragraph of the proof of $(4 \mathrm{G})$.

Let $(f, \sigma)=\left(f^{\prime}, \sigma^{\prime}\right)\left(f^{\prime \prime}, \sigma^{\prime \prime}\right) \in H$ with $\left(f^{\prime}, \sigma^{\prime}\right) \in\left(H^{\prime}\right)_{p^{\prime}}^{+}$and $\left(f^{\prime \prime}, \sigma^{\prime \prime}\right) \in\left(H^{\prime \prime}\right)^{+}$. If $\left(f^{\prime}, \sigma^{\prime}\right)$ or $\left(f^{\prime \prime}, \sigma^{\prime \prime}\right)$ is not in a splitting class of $H^{\prime}$ or $H^{\prime \prime}$, then $(f, \sigma)$ is not in a splitting class of $H$. Thus both sides of (4.10) are zero and (4.10) holds. So we may suppose $\left(f^{\prime}, \sigma^{\prime}\right)$ and $\left(f^{\prime \prime}, \sigma^{\prime \prime}\right)$ are in splitting classes of $H^{\prime}$ and $H^{\prime \prime}$. Suppose $\left(f^{\prime \prime}, \sigma^{\prime \prime}\right)$ is not in $\mathcal{C}_{\Lambda^{\prime \prime}}$. Then the right-hand side of (4.10) is zero by $(4 \mathrm{~F})$. Suppose the left-hand side of $(4.10)$ is non-zero. By $(4 \mathrm{H})$ (i) there exist $u^{\prime}$ in a $p$-regular splitting class of $H^{\prime}, u^{\prime \prime}$ in a $p$-singular splitting class of $H^{\prime \prime}$, and $h \in H^{+}$such that $\left(\left(f^{\prime}, \sigma^{\prime}\right)\left(f^{\prime \prime}, \sigma^{\prime \prime}\right)\right)^{h}=u^{\prime} u^{\prime \prime}$. Moreover, $u^{\prime \prime} \in \mathcal{C}_{\Lambda^{\prime \prime}}$. In particular, $u^{\prime \prime}$ is a product of cycles of $p$-singular length as an element of $S\left(\Gamma \times \Omega^{\prime \prime}\right)$. A comparison of the $p$ regular and $p$-singular cycle types of these elements as elements of $S(\Gamma \times \Omega)$ shows that $\left(f^{\prime}, \sigma^{\prime}\right)^{h}=u^{\prime},\left(f^{\prime \prime}, \sigma^{\prime \prime}\right)^{h}=u^{\prime \prime}$. Since $u^{\prime \prime}$ has no fixed-points on $\Omega^{\prime \prime}$, neither does $\left(f^{\prime \prime}, \sigma^{\prime \prime}\right)$. So $h$ stabilizes $\Omega^{\prime}$ and $\Omega^{\prime \prime}$, and $h \in H^{\prime} H^{\prime \prime}$. Thus $\left(f^{\prime}, \sigma^{\prime}\right)$ is conjugate to $u^{\prime}$ in $H^{\prime},\left(f^{\prime \prime}, \sigma^{\prime \prime}\right)$ is conjugate to $u^{\prime \prime}$ in $H^{\prime \prime}$, and $\left(f^{\prime \prime}, \sigma^{\prime \prime}\right) \in \mathcal{C}_{\Lambda^{\prime \prime}}$. This is a contradiction. So we may suppose $\left(f^{\prime}, \sigma^{\prime}\right)$ is in a $p$-regular splitting class of $H^{\prime}$ and $\left(f^{\prime \prime}, \sigma^{\prime \prime}\right)$ is in $\mathcal{C}_{\Lambda^{\prime \prime}}$.

Suppose there exists $(g, \tau)$ in $H^{+}$such that ${ }^{(g, \tau)}(f, \sigma)=\left(h^{\prime}, \rho^{\prime}\right)\left(h^{\prime \prime}, \rho^{\prime \prime}\right)$, where $\left(h^{\prime}, \rho^{\prime}\right) \in T_{\Lambda^{\prime}},\left(h^{\prime \prime}, \rho^{\prime \prime}\right) \in H_{\Lambda^{\prime \prime}}^{+}$, and

$$
\left(\Theta_{\Lambda^{\prime}}^{+}-\Theta_{\Lambda^{\prime}}^{-}\right)\left(h^{\prime}, \rho^{\prime}\right) \neq 0, \quad\left(\psi_{\Lambda^{\prime \prime},+}-\psi_{\Lambda^{\prime \prime},-}\right)\left(h^{\prime \prime}, \rho^{\prime \prime}\right) \neq 0
$$

If $\tau\left(i_{\delta}\right) \in \Omega^{\prime \prime}$ for some $i_{\delta} \in \Omega^{\prime}$, then $p_{\tau\left(i_{\delta}\right)}\left(h^{\prime \prime}, \rho^{\prime \prime}\right) \in a^{Y}$ by $(4 \mathrm{~F})$. But

$$
\begin{aligned}
p_{\tau\left(i_{\delta}\right)}\left(h^{\prime \prime}, \rho^{\prime \prime}\right) & =p_{\tau\left(i_{\delta}\right)}\left({ }^{(g, \tau)}(f, \sigma)\right) \\
& =g\left(\tau\left(i_{\delta}\right)\right) p_{i_{\delta}}(f, \sigma) g\left(\tau\left(i_{\delta}\right)\right)^{-1} \\
& =g\left(\tau\left(i_{\delta}\right)\right) p_{i_{\delta}}\left(f^{\prime}, \sigma^{\prime}\right) g\left(\tau\left(i_{\delta}\right)\right)^{-1}
\end{aligned}
$$

and $p_{i_{\delta}}\left(f^{\prime}, \sigma^{\prime}\right) \in\left(b^{i}\right)^{Y}$ for odd $i$ by hypothesis. This is impossible. Thus $\tau$ stabilizes $\Omega^{\prime}$ and $\Omega^{\prime \prime}$ and $(g, \tau)=\left(g^{\prime}, \tau^{\prime}\right)\left(g^{\prime \prime}, \tau^{\prime \prime}\right)$, where $\left(g^{\prime}, \tau^{\prime}\right) \in H^{\prime}$ and $\left(g^{\prime \prime}, \tau^{\prime \prime}\right) \in H^{\prime \prime}$. Then

$$
\begin{aligned}
& \left(\psi_{\Lambda,+}-\psi_{\Lambda,-}\right)(f, \sigma) \\
& \quad=\operatorname{Ind}_{T_{\Lambda^{\prime} \times H_{\Lambda^{\prime \prime}}^{+}}^{+}}^{H^{+}}\left(\left(\Theta_{\Lambda^{\prime}}^{+} \times \psi_{\Lambda^{\prime \prime},+}\right)-\left(\Theta_{\Lambda^{\prime}}^{+} \times \psi_{\Lambda^{\prime \prime},-}\right)\right)(f, \sigma) \\
& \quad=\operatorname{Ind}_{T_{\Lambda^{\prime}} \times H_{\Lambda^{\prime \prime}}^{+}}^{\left(H^{\prime} \times H^{\prime \prime}\right.}\left(\left(\Theta_{\Lambda^{\prime}}^{+} \times \psi_{\Lambda^{\prime \prime},+}\right)-\left(\Theta_{\Lambda^{\prime}}^{+} \times \psi_{\Lambda^{\prime \prime},-}\right)\right)(f, \sigma) .
\end{aligned}
$$


Let $\mathcal{D}$ be a transversal for $T_{\Lambda^{\prime}}$ in $\left(H^{\prime}\right)^{+}$. Then $\mathcal{D} \cup \mathcal{D} s$ is a transversal for $T_{\Lambda^{\prime}} \times H_{\Lambda^{\prime \prime}}^{+}$ in $\left(H^{\prime} \times H^{\prime \prime}\right)^{+}$, where $s=(h, 1)$ is the element introduced earlier in the proof. It follows that

$$
\begin{aligned}
& \left(\psi_{\Lambda,+}-\psi_{\Lambda,-}\right)(f, \sigma) \\
& =\operatorname{Ind}_{T_{\Lambda^{\prime}}}^{\left(H^{\prime}\right)^{+}}\left(\Theta_{\Lambda^{\prime}}^{+}\right)\left(f^{\prime}, \sigma^{\prime}\right) \times\left(\psi_{\Lambda^{\prime \prime},+}-\psi_{\Lambda^{\prime \prime},-}\right)\left(f^{\prime \prime}, \sigma^{\prime \prime}\right) \\
& \quad \quad \quad \operatorname{Ind}_{\left.T_{\Lambda^{\prime}}\right)^{+}}^{\left(H^{\prime}\right.}\left(\Theta_{\Lambda^{\prime}}^{-}\right)\left(f^{\prime}, \sigma^{\prime}\right) \times\left(\psi_{\Lambda^{\prime \prime},-}-\psi_{\Lambda^{\prime \prime},+}\right)\left(f^{\prime \prime}, \sigma^{\prime \prime}\right) \\
& =\left(\psi_{\Lambda^{\prime},+}-\psi_{\Lambda^{\prime},-}\right)\left(f^{\prime}, \sigma^{\prime}\right) \times\left(\psi_{\Lambda^{\prime \prime},+}-\psi_{\Lambda^{\prime \prime},-}\right)\left(f^{\prime \prime}, \sigma^{\prime \prime}\right),
\end{aligned}
$$

and (4.10) holds. Suppose no $(g, \tau)$ in $H^{+}$satisfies $(4.14)$. Then $\left(\psi_{\Lambda,+}-\psi_{\Lambda,-}\right)(f, \sigma)$ is 0 by (4.12). Thus (4.10) holds unless

$$
\left(\psi_{\Lambda^{\prime},+}-\psi_{\Lambda^{\prime},-}\right)\left(f^{\prime}, \sigma^{\prime}\right) \neq 0, \quad\left(\psi_{\Lambda^{\prime \prime},+}-\psi_{\Lambda^{\prime \prime},-}\right)\left(f^{\prime \prime}, \sigma^{\prime \prime}\right) \neq 0 .
$$

In this case there exists $\left(g^{\prime}, \tau^{\prime}\right)$ in $\left(H^{\prime}\right)^{+}$such that ${ }^{\left(g^{\prime}, \tau^{\prime}\right)}\left(f^{\prime}, \sigma^{\prime}\right)=\left(h^{\prime}, \rho^{\prime}\right)$ is in $T_{\Lambda^{\prime}}$

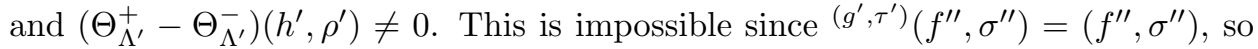
that (4.14) holds. This completes the proof of $(4 \mathrm{H})$, (ii).

Corollary (4I). Let $\Lambda$ be a splitting mapping of $H$. If $\left(\psi_{\Lambda,+}-\psi_{\Lambda,-}\right)(f, \sigma) \neq 0$, then

$$
\left(\psi_{\Lambda,+}-\psi_{\Lambda,-}\right)(f, \sigma) \equiv 0\left(\bmod \sqrt{\left|C_{H}(f, \sigma)\right|_{p}}\right)
$$

and $\left|C_{H}(f, \sigma)\right|_{p}=p^{d}$, where $d$ is the number of diagonal nodes in $\lambda_{p^{*}}$.

Proof. This follows from $(4 \mathrm{H}),(4 \mathrm{~F})$, and (4.3).

\section{The isotypy CONJECTURE FOR ALternating GRoups For $p>2$}

We suppose $p$ is odd in this section. Let $G$ and $G^{+}$be the symmetric and alternating groups on the set $\Pi$ of $n$ elements. Let $e$ and $e^{+}$be blocks of $\mathcal{O} G$ and $\mathcal{O} G^{+}$such that $e$ covers $e^{+}$. In particular, $e$ and $e^{+}$have the same defect group $D$. If $D$ is non-trivial, then there exists $\chi_{\lambda}$ in $(G, e)^{\vee}$ such that $\operatorname{Res}_{G^{+}}^{G}\left(\chi_{\lambda}\right)$ is irreducible. Indeed, let $\lambda_{0}$ be the $p$-core parametrizing $e$, and choose a partition $\lambda$ of $n$ with $p$-core $\lambda_{0}$ and a $p$-quotient $\Lambda$ such that $\Lambda^{*} \neq \Lambda$. In particular, $e^{+}$is the unique block of $\mathcal{O} G^{+}$covered by $e$.

Let $H=N_{G}(D)$ and $H^{+}=N_{G^{+}}(D)$. Let $f=\operatorname{Br}_{D}^{G}(e)$ and $f^{+}=\operatorname{Br}_{D}^{G^{+}}\left(e^{+}\right)$be the Brauer correspondents of $e$ and $e^{+}$in $\mathcal{O H}$ and $\mathcal{O H}^{+}$. In particular, $f^{+}$is $H$-stable since $e^{+}$is $G$-stable. Moreover, $f f^{+}=f$, since $e e^{+}=e$ and $\operatorname{Br}_{D}^{G}\left(e^{+}\right)=\operatorname{Br}_{D}^{G^{+}}\left(e^{+}\right)$. Thus $f$ covers $f^{+}$, and $f^{+}$is the unique block of $\mathcal{O H}^{+}$covered by $f$. We suppose from now on that $D$ is abelian and that $|D|=p^{w}$ with $1 \leq w<p$. Then $e$ and $f$ are isotypic blocks by (3B). If $\lambda_{0}^{*} \neq \lambda_{0}$, then $e^{+}$and $f^{+}$are isotypic blocks by (1E). So we also suppose from now on that $\lambda_{0}^{*}=\lambda_{0}$. Thus $e^{*}=e=e^{+}, f^{*}=f=f^{+}$, and $\left(G, G^{+}, e, e^{+}\right)$and $\left(H, H^{+}, f, f^{+}\right)$are +configurations.

We use the notation of $\S 3: \Pi=\Pi_{0} \cup \Pi_{1}$, where $\Pi_{0}$ and $\Pi_{1}$ are disjoint sets of $n_{0}$ and $p w$ elements; $\Pi_{1}=\Gamma \times \Omega$, where $\Gamma=\{1,2, \ldots, p\}$ and $|\Omega|=w ; X=S(\Gamma)$ and $Y=N_{X}(P)$, where $P$ is a Sylow $p$-subgroup of $X$; and $D$ is the Sylow $p$-subgroup $P^{\Omega}$ of $S\left(\Pi_{1}\right)$. Let

$$
\begin{aligned}
H_{0} & =S\left(\Pi_{0}\right), \quad H_{1}=Y \nmid S(\Omega), \\
H_{0}^{+} & =A\left(\Pi_{0}\right), \quad H_{1}^{+}=(Y \backslash S(\Omega))^{+},
\end{aligned}
$$


and set $H^{++}=H_{0}^{+} \times H_{1}^{+}$. Then $H=H_{0} \times H_{1}, H>H^{+} \geq H^{++}$, and $H^{+} / H^{++}$ has order 1 or 2 according as $\left|\Pi_{0}\right| \leq 1$ or $\left|\Pi_{0}\right|>1$. We have

$$
f^{+}=f=e_{\lambda_{0}} \times 1_{H_{1}},
$$

where $e_{\lambda_{0}}$ is the block of defect 0 of $\mathcal{O} H_{0}$ parametrized by $\lambda_{0}$. Let $\chi_{\lambda_{0}}$ denote the character in $\left(H_{0}, e_{\lambda_{0}}\right)^{\vee}$. Since $\lambda_{0}$ is self-dual, either $\left|\lambda_{0}\right| \leq 1$ or $\left|\lambda_{0}\right| \geq 3$. It will be convenient to interpret $H_{0}$ and $H_{0}^{+}$as the identity group when $\left|\lambda_{0}\right| \leq 1$ and to omit identity factors in expressions such as (5.2). We fix a maximal $e^{+}$-subpair $\left(D, e_{D}^{+}\right)$ and a maximal $f^{+}$-subpair $\left(D, f_{D}^{+}\right)$as follows: $C_{G^{+}}(D)=C_{H^{+}}(D)=H_{0}^{+} \times D$. If $\left|\Pi_{0}\right| \leq 1$, let $e_{D}^{+}=f_{D}^{+}$be the 1-block; if $\left|\Pi_{0}\right| \geq 3$, let $e_{D}^{+}=f_{D}^{+}=e_{\lambda_{0},+}$, where $e_{\lambda_{0},+}$ is the block of $\mathcal{O} H_{0}^{+}$containing an irreducible constituent $\chi_{\lambda_{0},+}$ of $\operatorname{Res}_{H_{0}^{+}}^{H_{0}}\left(\chi_{\lambda_{0}}\right)$. In both cases

$$
N_{G^{+}}\left(D, e_{D}^{+}\right)=N_{H^{+}}\left(D, f_{D}^{+}\right)=H^{++} .
$$

We describe certain subpairs in $\mathbf{B r}_{e^{+}}\left(G^{+}\right)$and $\mathbf{B r}_{f^{+}}\left(H^{+}\right)$. Every $e^{+}$-subpair $\left(Q, u^{+}\right)$is covered by an $e$-subpair $(Q, u)$ in the sense that $u$ covers $u^{+}$. Conversely, any subpair $\left(Q, u^{+}\right)$of $G^{+}$covered by an $e$-subpair $(Q, u)$ is an $e^{+}$-subpair. Indeed, these claims follow from the relation $\operatorname{Br}_{Q}^{G}(e)=\operatorname{Br}_{Q}^{G^{+}}\left(e^{+}\right)$. If $\left|\Pi_{0}\right| \leq 1$, then $e^{+}$ and $f^{+}$are the 1-blocks of $\mathcal{O} G^{+}$and $\mathcal{O} H^{+}$, and Brauer's Third Main Theorem implies the subpairs in $\mathbf{B r}_{e^{+}}\left(G^{+}\right)$and $\mathbf{B r}_{f^{+}}\left(H^{+}\right)$are $\left(Q, e_{Q}^{+}\right)$and $\left(Q, f_{Q}^{+}\right)$, where $e_{Q}^{+}$and $f_{Q}^{+}$are the 1-blocks of $\mathcal{O} G_{Q}^{+}$and $\mathcal{O} H_{Q}^{+}$. So we suppose $\left|\Pi_{0}\right| \geq 3$. Let $x$ be a standard non-identity element of $D$ in the sense of $\S 3$, and recall that

$$
G_{x}=G_{x, 0} \times G_{x, 1}, \quad H_{x}=H_{x, 0} \times H_{x, 1},
$$

where $G_{x, 0}=S\left(\Pi_{0} \cup\left(\Gamma \times \Omega_{x, 0}\right)\right), H_{x, 0}=S\left(\Pi_{0}\right) \times\left(Y \backslash S\left(\Omega_{x, 0}\right)\right)$, and $G_{x, 1}=H_{x, 1}=$ $P\left(S\left(\Omega_{x, 1}\right)\right.$. Let $H_{x, 0}=H_{x, 00} \times H_{x, 01}$, where $H_{x, 00}=S\left(\Pi_{0}\right)$ and $H_{x, 01}=Y \imath S\left(\Omega_{x, 0}\right)$. Let

$$
e_{x}=e_{x, 0} \times e_{x, 1}, \quad f_{x}=f_{x, 00} \times f_{x, 01} \times f_{x, 1},
$$

where $e_{x, 0}$ is the block of $\mathcal{O} G_{x, 0}$ parametrized by $\lambda_{0}, e_{x, 1}$ is the 1-block of $\mathcal{O} G_{x, 1}$, $f_{x, 00}$ is the block of $\mathcal{O} H_{x, 00}$ parametrized by $\lambda_{0}, f_{x, 01}$ is the 1-block of $\mathcal{O} H_{x, 01}$, and $f_{x, 1}$ is the 1-block of $\mathcal{O} H_{x, 1}$. Let $f_{x, 0}$ be the block $f_{x, 00} \times f_{x, 01}$ of $\mathcal{O} H_{x, 0}$. Let $G_{x}^{+}$and $H_{x}^{+}$be the intersections of $G_{x}$ and $H_{x}$ with $A(\Pi)$. Similarly, let $G_{x, 0}^{+}$and $H_{x, 0}^{+}$be the intersections of $G_{x, 0}$ and $H_{x, 0}$ with $A\left(\Pi_{0} \cup\left(\Gamma \times \Omega_{x, 0}\right)\right)$, and let $G_{x, 1}^{+}$ and $H_{x, 1}^{+}$be the intersections of $G_{x, 1}$ and $H_{x, 1}$ with $A\left(\Gamma \times \Omega_{x, 1}\right)$. In particular, $G_{x}>G_{x}^{+}$if $\left|\Pi_{0}\right|>1$ or $w>1$. Let $G_{x}^{++}=G_{x, 0}^{+} \times G_{x, 1}^{+}$and $H_{x}^{++}=H_{x, 0}^{+} \times H_{x, 1}^{+}$. If $w>1$, then $e_{x}$ and $f_{x}$ cover unique blocks $e_{x}^{+}$and $f_{x}^{+}$of $\mathcal{O} G_{x}^{+}$and $\mathcal{O} H_{x}^{+}$. Indeed, let $\chi_{x}=\chi_{x, 0} \times \chi_{x, 1}$, where $\chi_{x, 0} \in\left(G_{x, 0}, e_{x, 0}\right)^{\vee}, \chi_{x, 1} \in\left(G_{x, 1}, e_{x, 1}\right)^{\vee}$, and $\chi_{x, 0}$ or $\chi_{x, 1}$ is irreducible on $G_{x, 0}^{+}$or $G_{x, 1}^{+}$. Such a $\chi_{x, 0}$ or $\chi_{x, 1}$ exists since $\left|\Omega_{x, 0}\right|>0$ or $\left|\Omega_{x, 1}\right| \geq 2$. So $\chi_{x} \in\left(G_{x}, e_{x}\right)^{\vee}, \chi_{x}$ is irreducible on $G_{x}^{+}$, and $e_{x}$ covers a unique block $e_{x}^{+}$of $\mathcal{O} G_{x}^{+}$. A similar argument shows that $f_{x}$ covers a unique block $f_{x}^{+}$of $\mathcal{O} H_{x}^{+}$.

Proposition (5A). Let $x$ be a non-identity standard element in D. If $\left|\Pi_{0}\right| \leq 1$ or $w>1$, then $\operatorname{Br}_{\langle x\rangle}^{G^{+}}\left(e^{+}\right)$and $\operatorname{Br}_{\langle x\rangle}^{H^{+}}\left(f^{+}\right)$are blocks of $\mathcal{O} G_{x}^{+}$and $\mathcal{O} H_{x}^{+}$. If $\left|\Pi_{0}\right| \geq 3$ and $w=1$, then $D=\langle x\rangle, G_{x}^{+}=H_{x}^{+}=H_{0}^{+} \times D$, and

$$
\operatorname{Br}_{\langle x\rangle}^{G^{+}}\left(e^{+}\right)=\operatorname{Br}_{\langle x\rangle}^{H^{+}}\left(f^{+}\right)=e_{\lambda_{0},+}+e_{\lambda_{0},-} .
$$


Proof. If $\left|\Pi_{0}\right| \leq 1$, then $e^{+}$and $f^{+}$are the principal blocks and (5A) is clear. If $\left|\Pi_{0}\right| \geq 3$ and $w>1$, then (5A) follows from the preceding paragraph and the fact that $e$ and $f$ are blocks of principal type. If $\left|\Pi_{0}\right| \geq 3$ and $w=1$, then the result follows from our discussion of the maximal $e^{+}$-subpair $\left(D, e_{D}^{+}\right)$and the maximal $f^{+}$-subpair $\left(D, f_{D}^{+}\right)$.

Proposition (5B). Let $\mu=(J, \epsilon)$ be the Rouquier isometry in $\mathcal{R}_{K}((G, e),(H, f))$. Then the following hold:

(i) $\mu$ is +equivariant with respect to $\left(G^{+}, H^{+}\right)$.

(ii) Every $\mu$-induced isometry $\mu^{+}=\left(\mathrm{J}^{+}, \epsilon^{+}\right)$in $\mathcal{R}_{K}\left(\left(G^{+}, e^{+}\right),\left(H^{+}, f^{+}\right)\right)$is perfect.

Proof. By definition (3.8) of the Rouquier isometry

$$
\mathrm{J}\left(\operatorname{sgn}_{G} \chi_{\lambda}\right)=\mathrm{J}\left(\chi_{\lambda^{*}}\right)=\chi_{\lambda_{0}^{*}} \times \psi_{\widehat{\Lambda^{*}}}=\operatorname{sgn}_{H_{0}} \chi_{\lambda_{0}} \times \operatorname{sgn}_{H_{1}} \psi_{\widehat{\Lambda}}=\operatorname{sgn}_{H} \mathrm{~J}\left(\chi_{\lambda}\right) .
$$

Moreover, $\left.\epsilon\left(\operatorname{sgn}_{G} \chi_{\lambda}\right)\right)=\epsilon\left(\chi_{\lambda}\right)$, since $\left|\lambda_{\frac{1}{2}(p+1)}\right|=\left|\lambda_{\frac{1}{2}(p+1)}^{*}\right|$ and $v(\lambda)=v\left(\lambda^{*}\right)$. Here the last equality holds since the dual of a sequence of $p$-hooks leading from $\lambda$ to $\lambda_{0}$ is a sequence of $p$-hooks from $\lambda^{*}$ to $\lambda_{0}^{*}$, and the leg and arm lengths of a $p$-hook have the same parity.

For (ii) it suffices by (2B) to show that

$$
\Phi_{\lambda}(g, h)=\left(\chi_{\lambda,+}-\chi_{\lambda,-}\right)(g)\left(\mathrm{J}^{+}\left(\chi_{\lambda,+}\right)-\mathrm{J}^{+}\left(\chi_{\lambda,-}\right)\right)\left(h^{-1}\right)
$$

is perfect on $G^{+} \times H^{+}$for all self-dual partitions $\lambda$ with $p$-core $\lambda_{0}$. Suppose $\Phi_{\lambda}(g, h) \neq 0$ for $g \in G^{+}, h \in H^{+}$. Then $g$ and $h$ are in splitting classes of $G$ and $H$, and

$$
\left(\chi_{\lambda,+}-\chi_{\lambda,-}\right)(g) \neq 0, \quad\left(\mathrm{~J}^{+}\left(\chi_{\lambda,+}\right)-\mathrm{J}^{+}\left(\chi_{\lambda,-}\right)\right)\left(h^{-1}\right) \neq 0 .
$$

The first inequality and (4A) imply that $g$ has cycle type $\eta(\lambda)$. If $d$ is the number of $\eta_{i i}(\lambda)$ divisible by $p$, then

$$
\left(\chi_{\lambda,+}-\chi_{\lambda,-}\right)(g) \equiv 0\left(\bmod \sqrt{\epsilon_{\lambda} p^{d}}\right)
$$

and $\left|C_{G}(g)\right|_{p}=p^{d}$. Moreover, $g$ is $p$-singular if and only if $d>0$. The second inequality implies that $h \in H^{++}$. Indeed, if $\left|\Pi_{0}\right| \leq 1$, then $h \in H^{++}$since $H^{+}=$ $H^{++}$. If $\left|\Pi_{0}\right| \geq 3$, then $\chi_{\lambda_{0}}$ and $\psi_{\widehat{\Lambda}}$ are reducible on $H_{0}^{+}$and $H_{1}^{+}$, and $\mathrm{J}^{+}\left(\chi_{\lambda, \pm}\right)$ has support on $H^{++}$. Thus $h=h_{0}\left(f_{1}, \sigma_{1}\right)$, where $h_{0} \in H_{0}^{+},\left(f_{1}, \sigma_{1}\right) \in H_{1}^{+}$, and

$$
\left(\chi_{\lambda_{0},+}-\chi_{\lambda_{0},-}\right)\left(h_{0}^{-1}\right) \neq 0, \quad\left(\psi_{\widehat{\Lambda}_{,}+}-\psi_{\widehat{\Lambda},-}\right)\left(\left(f_{1}, \sigma_{1}\right)^{-1}\right) \neq 0 .
$$

Here $\left(\chi_{\lambda_{0},+}-\chi_{\lambda_{0},-}\right)\left(h_{0}^{-1}\right)$ is interpreted as 1 if $\left|\Pi_{0}\right| \leq 1$. Since $\chi_{\lambda_{0}, \pm}$ has defect 0 , it follows that $h_{0}$ is $p$-regular and $\left(\chi_{\lambda_{0},+}-\chi_{\lambda_{0},-}\right)\left(h_{0}\right)$ is divisible by $\left|C_{H_{0}}\left(h_{0}\right)\right|_{p}$. On the other hand, (4I) implies that $\left|C_{H_{1}}\left(f_{1}, \sigma_{1}\right)\right|_{p}=p^{d}$ and

$$
\left(\psi_{\widehat{\Lambda},+}-\psi_{\widehat{\Lambda},-}\right)\left(f_{1}, \sigma_{1}\right) \equiv 0\left(\bmod \sqrt{\left|C_{H_{1}}\left(f_{1}, \sigma_{1}\right)\right|_{p}}\right) .
$$

Moreover, $h$ is $p$-singular if and only if $d>0$. It now follows that $\Phi_{\lambda}$ is perfect.

For each $j=1,2, \ldots, w$, let $x_{j}$ be a standard element of $D$ such that $\left|\Omega_{x_{j}, 1}\right|=j$. We claim the $w$ subgroups $\left\langle x_{j}\right\rangle$ form a set of representatives for the $N_{G^{+}}\left(D, e_{D}^{+}\right)$orbits of non-trivial cyclic subgroups of $D$. This is trivially true when $w=1$, so we may suppose $w>1$. The $w$ subgroups $\left\langle x_{j}\right\rangle$ form a set of representatives for the 
$(Y \nmid S(\Omega))$-orbits of non-trivial cyclic subgroups of $D$. But the $(Y \nmid S(\Omega))$-orbit of $\left\langle x_{j}\right\rangle$ is the $\left(Y\langle S(\Omega))^{+}\right.$-orbit of $\left\langle x_{j}\right\rangle$, since

$$
C_{Y \backslash S(\Omega)}\left(x_{j}\right)=\left(Y 2 S\left(\Omega_{x_{j}, 0}\right)\right) \times\left(P \prec S\left(\Omega_{x_{j}, 1}\right)\right)
$$

contains odd permutations when $w>1$. Since $(Y \backslash S(\Omega))^{+} \leq N_{G^{+}}\left(D, e_{D}^{+}\right)$, the claim holds.

We will use the isotypy (3B) between $e$ and $f$ to define an isometry $\mu^{+}$and perfect isometries $\mu_{x}^{+}$for $x$ in $\left\{x_{1}, x_{2}, \ldots, x_{w}\right\}$ so that the diagrams

$$
\begin{array}{rrr}
\mathcal{R}_{K}\left(G^{+}, e^{+}\right) & \stackrel{\mathrm{R}_{\mu^{+}}}{\longrightarrow} & \mathcal{R}_{K}\left(H^{+}, f^{+}\right) \\
\downarrow d_{G^{+}}^{x, e_{x}^{+}} & & \downarrow d_{H^{+}}^{x, f_{x}^{+}} \\
\mathrm{CF}_{p^{\prime}}\left(G_{x}^{+}, e_{x}^{+}\right) & \stackrel{\mathrm{R}_{\mu_{x}^{+}, p^{\prime}}}{\longrightarrow} & \mathrm{CF}_{p^{\prime}}\left(H_{x}^{+}, f_{x}^{+}\right)
\end{array}
$$

are commutative. The definitions require a consistent labeling of splitting characters and splitting classes.

The following three configurations (5.6), (5.7), (5.8) are basic for the labeling:

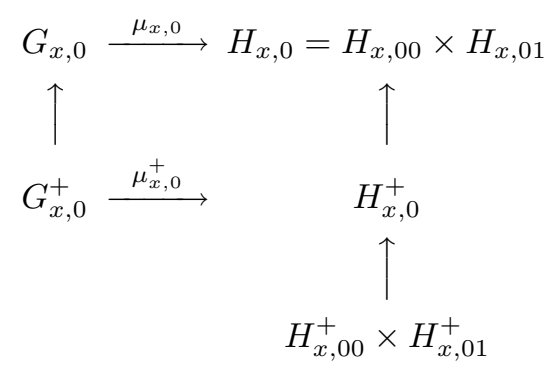

where $\mu_{x, 0}$ is the Rouquier isometry. If $G_{x, 0}=G_{x, 0}^{+}$or $G_{x, 0}=H_{x, 0}$, then $\mu_{x, 0}$ is the identity isometry. Here $G_{x, 0}=G_{x, 0}^{+}$if $\left|\Pi_{0}\right| \leq 1$ and $j=w ; G_{x, 0}=H_{x, 0}$ if $j=w$. In these cases, we fix $\mu_{x, 0}^{+}$as the identity isometry. If $G_{x, 0}>G_{x, 0}^{+}$, then $\left(G_{x, 0}, G_{x, 0}^{+}, e_{x, 0}, e_{x, 0}^{+}\right)$and $\left(H_{x, 0}, H_{x, 0}^{+}, f_{x, 0}, f_{x, 0}^{+}\right)$are +configurations, and $\mu_{x, 0}$ is +equivariant with respect to $\left(G_{x, 0}^{+}, H_{x, 0}^{+}\right)$by $(5 \mathrm{~B})$, (i). We will fix $\mu_{x, 0}^{+}$in what follows so that $\mu_{x, 0}^{+}$is $\mu_{x, 0}$-induced and hence perfect by (5B), (ii).

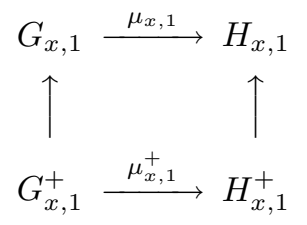

where $\mu_{x, 1}$ is the identity isometry. We fix $\mu_{x, 1}^{+}$as the identity isometry. If $G_{x, 1}>$ $G_{x, 1}^{+}$, then $\left(G_{x, 1}, G_{x, 1}^{+}, e_{x, 1}, e_{x, 1}^{+}\right)$and $\left(H_{x, 1}, H_{x, 1}^{+}, f_{x, 1}, f_{x, 1}^{+}\right)$are +configurations, $\mu_{x, 1}$ is trivially +equivariant with respect to $\left(G_{x, 1}^{+}, H_{x, 1}^{+}\right)$, and $\mu_{x, 1}^{+}$is trivially $\mu_{x, 1^{-}}$ induced. Here $G_{x, 1}>G_{x, 1}^{+}$if and only if $j>1$. 


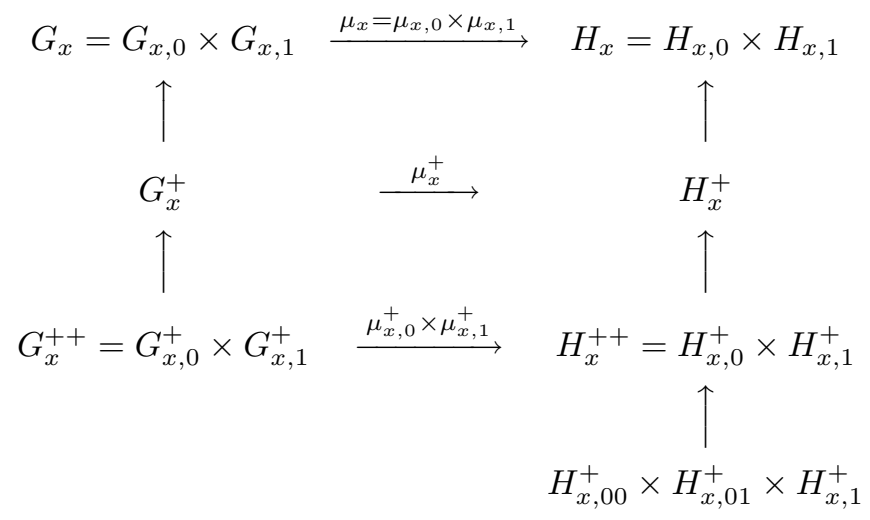

where $\mu_{x, 0}, \mu_{x, 1}, \mu_{x, 0}^{+}, \mu_{x, 1}^{+}$are the perfect isometries in (5.6) and (5.7). If $G_{x}=G_{x}^{+}$, then $G_{x, 0}=G_{x, 0}^{+}$and $G_{x, 1}=G_{x, 1}^{+}$, that is, $\left|\Pi_{0}\right| \leq 1$ and $w=1$. Thus $G_{x}=G_{x}^{+}=$ $G_{x}^{++}$and $\mu_{x}$ is the identity isometry. In this case we fix $\mu_{x}^{+}$as the identity isometry. If $G_{x}>G_{x}^{+}$and $G_{x}^{+}=G_{x}^{++}$, then either $G_{x, 0}=G_{x, 0}^{+}$or $G_{x, 1}=G_{x, 1}^{+}$, that is, either $\left|\Pi_{0}\right| \leq 1$ and $1<j=w$, or $\left|\Pi_{0} \cup\left(\Gamma \times \Omega_{x, 0}\right)\right| \geq 3$ and $j=1$. In this case we fix $\mu_{x}^{+}=\mu_{x, 0}^{+} \times \mu_{x, 1}^{+}$. If $G_{x}>G_{x}^{+}>G_{x}^{++}$, then

$$
\begin{array}{ll}
\left(G_{x}, G_{x}^{+}, e_{x}, e_{x}^{+}\right), & \left(H_{x}, H_{x}^{+}, f_{x}, f_{x}^{+}\right), \\
\left(G_{x}^{+}, G_{x}^{++}, e_{x}^{+}, e_{x}^{++}\right), & \left(H_{x}^{+}, H_{x}^{++}, f_{x}^{+}, f_{x}^{++}\right)
\end{array}
$$

are +configurations. Moreover, $\mu_{x}$ is perfect and +equivariant with respect to $\left(G_{x}^{+}, H_{x}^{+}\right)$by $(2 \mathrm{E}),(\mathrm{i})$. We will fix $\mu_{x}^{+}$in what follows so that $\mu_{x}^{+}$is $\mu_{x}$-induced and so that $\mu_{x, 0}^{+} \times \mu_{x, 1}^{+}$is $\mu_{x}^{+}$-induced. This is possible by $(2 \mathrm{~F})$, (i). In particular, $\mu_{x}^{+}$is perfect by (5B), (ii).

We fix $\mu_{x}^{+}$for $x=x_{j}$ in the remaining cases as follows:

Step 1. If $\left|\lambda_{0}\right| \geq 3$, then the character $\chi_{\lambda_{0}}$ of $H_{x, 00}$ splits on $H_{x, 00}^{+}$. We label the irreducible characters $\chi_{\lambda_{0}, \pm}$ and the conjugacy classes $\mathcal{C}_{\eta\left(\lambda_{0}\right), \pm}$ of $H_{x, 00}^{+}$so that $(4 \mathrm{~A})$ holds. This labeling is fixed for all $j$. If $\left|\lambda_{0}\right| \leq 1$, then $H_{x, 00}=H_{x, 00}^{+}=1$ and we omit this step.

Step 2. If $\left|\Omega_{x, 0}\right| \geq 1$, let $\Lambda^{\prime}$ run over the $p$-regular splitting mappings of $H_{x, 01}$. The splitting characters $\psi_{\Lambda^{\prime}}$ of $H_{x, 01}$ satisfy the hypotheses of $(4 \mathrm{G})$; the splitting classes $\mathcal{C}_{\Lambda^{\prime}}$ of $H_{x, 01}$ have $p$-regular cycle type. We label arbitrarily the irreducible characters $\psi_{\Lambda^{\prime}, \pm}$ of $H_{x, 01}^{+}$and the conjugacy classes $\mathcal{C}_{\Lambda^{\prime}, \pm}$ of $H_{x, 01}^{+}$. If $\left|\Omega_{x, 0}\right|=0$, then $H_{x, 01}=H_{x, 01}^{+}=1$ and we omit this step.

Step 3. Let $\Lambda^{\prime \prime}$ run over the $p$-singular splitting mappings of $Y \backslash S\left(\Omega_{x, 1}\right)$. Such mappings exist since $\left|\Omega_{x, 1}\right| \geq 1$. The splitting characters $\psi_{\Lambda^{\prime \prime}}$ of $Y \backslash S\left(\Omega_{x, 1}\right)$ satisfy the hypotheses of $(4 \mathrm{~F})$; the splitting classes $\mathcal{C}_{\Lambda^{\prime \prime}}$ of $Y 2 S\left(\Omega_{x, 1}\right)$ have $p$-singular cycle type. We label the irreducible characters $\psi_{\Lambda^{\prime \prime}, \pm}$ of $Y \backslash S\left(\Omega_{x, 1}\right)$ and the conjugacy classes $\mathcal{C}_{\Lambda^{\prime \prime}, \pm}$ of $Y \nmid S\left(\Omega_{x, 1}\right)$ so that $(4 \mathrm{~F})$ holds.

Step 4. Let $\lambda^{\prime}$ run over all self-dual partitions of $\left|\Pi_{0} \cup\left(\Gamma \times \Omega_{x, 0}\right)\right|$ with $p$-core $\lambda_{0}$ and $p$-regular $p$-quotient $\Lambda^{\prime}$. Suppose $\left|\Omega_{x, 0}\right|=0$. Then $\Lambda^{\prime}$ is trivial, $\lambda^{\prime}=\lambda_{0}$, $G_{x, 0}=H_{x, 0}=H_{x, 00}$, and $\chi_{\lambda^{\prime}, \pm}$ and $\mathcal{C}_{\eta\left(\lambda^{\prime}\right), \pm}$ have already been labeled in step 1. Suppose $\left|\Omega_{x, 0}\right| \geq 1$. Then $\Lambda^{\prime}$ is a $p$-regular splitting mapping of $H_{x, 01}$ of the type 
considered in step 2. The conjugacy class $\mathcal{C}_{\eta\left(\lambda^{\prime}\right)}$ of $G_{x, 0}$ contains the conjugacy class $\mathcal{C}_{\eta\left(\lambda_{0}\right)} \mathcal{C}_{\Lambda^{\prime}}$ of $H_{x, 0}$. If $\left|\lambda_{0}\right| \geq 3$, we label $\mathcal{C}_{\eta\left(\lambda^{\prime}\right), \pm}$ so that

$$
\begin{aligned}
& \mathcal{C}_{\eta\left(\lambda^{\prime}\right),+}=\left(\mathcal{C}_{\eta\left(\lambda_{0}\right),+} \mathcal{C}_{\Lambda^{\prime},+}\right)^{G_{x, 0}^{+}}=\left(\mathcal{C}_{\eta\left(\lambda_{0}\right),-} \mathcal{C}_{\Lambda^{\prime},-}\right)^{G_{x, 0}^{+}}, \\
& \mathcal{C}_{\eta\left(\lambda^{\prime}\right),-}=\left(\mathcal{C}_{\eta\left(\lambda_{0}\right),+} \mathcal{C}_{\Lambda^{\prime},-}\right)^{G_{x, 0}^{+}}=\left(\mathcal{C}_{\eta\left(\lambda_{0}\right),-} \mathcal{C}_{\Lambda^{\prime},+}\right)^{G_{x, 0}^{+}} .
\end{aligned}
$$

If $\left|\lambda_{0}\right| \leq 1$, then $H_{x, 00}=H_{x, 00}^{+}=1$ and we label $\mathcal{C}_{\eta\left(\lambda^{\prime}\right), \pm}$ so that $\mathcal{C}_{\eta\left(\lambda^{\prime}\right),+}=$ $\left(\mathcal{C}_{\Lambda^{\prime},+}\right)^{G_{x, 0}^{+}}$and $\mathcal{C}_{\eta\left(\lambda^{\prime}\right),-}=\left(\mathcal{C}_{\Lambda^{\prime},-}\right)^{G_{x, 0}^{+}}$. We then label the irreducible characters $\chi_{\lambda^{\prime}, \pm}$ of $G_{x, 0}^{+}$so that $(4 \mathrm{~A})$ holds. We label the irreducible characters $\left(\chi_{\lambda_{0}} \psi_{\Lambda^{\prime}}\right)_{ \pm}$of $H_{x, 0}^{+}$so that

$$
\begin{gathered}
\operatorname{Res}_{H_{x, 00}^{+} H_{x, 01}^{+}}^{H_{x, 0}^{+}}\left(\left(\chi_{\lambda_{0}} \psi_{\Lambda^{\prime}}\right)_{+}\right)=\chi_{\lambda_{0},+} \psi_{\Lambda^{\prime},+}+\chi_{\lambda_{0},-} \psi_{\Lambda^{\prime},-}, \\
\operatorname{Res}_{H_{x, 00}^{+} H_{x, 01}^{+}}^{H_{x}^{+}}\left(\left(\chi_{\lambda_{0}} \psi_{\Lambda^{\prime}}\right)_{-}\right)=\chi_{\lambda_{0},+} \psi_{\Lambda^{\prime},-}+\chi_{\lambda_{0},-} \psi_{\Lambda^{\prime},+}
\end{gathered}
$$

for $\left|\lambda_{0}\right| \geq 3$, and so that

$$
\left(\chi_{\lambda_{0}} \psi_{\Lambda^{\prime}}\right)_{+}=1_{S\left(\Pi_{0}\right)} \times \psi_{\Lambda^{\prime},+}, \quad\left(\chi_{\lambda_{0}} \psi_{\Lambda^{\prime}}\right)_{-}=1_{S\left(\Pi_{0}\right)} \times \psi_{\Lambda^{\prime},-}
$$

for $\left|\lambda_{0}\right| \leq 1$.

Step 5. Let $\mu_{x, 0}=\left(\mathrm{J}_{x, 0}, \epsilon_{x, 0}\right)$. We fix a $\mu_{x, 0}$-induced isometry $\mu_{x, 0}^{+}=\left(\mathrm{J}_{x, 0}^{+}, \epsilon_{x, 0}^{+}\right)$so that

$$
\mathrm{J}_{x, 0}^{+}\left(\chi_{\lambda^{\prime}, \pm}\right)=\mathrm{J}_{x, 0}\left(\chi_{\lambda^{\prime}}\right)_{ \pm}=\left(\chi_{\lambda_{0}} \psi_{\Lambda^{\prime}}\right)_{ \pm}
$$

for the $\lambda^{\prime}$ in step 4 . Here $\widehat{\Lambda^{\prime}}=\Lambda^{\prime}$, since $\Lambda^{*}=\Lambda$.

Step 6. We label arbitrarily the irreducible characters and the conjugacy classes of $G_{x, 1}^{+}=H_{x, 1}^{+}$occurring in splitting irreducible characters and conjugacy classes of $G_{x, 1}=H_{x, 1}$. Here $\mu_{x, 1}^{+}$was already chosen as the identity isometry. If $G_{x}>G_{x}^{+}>$ $G_{x}^{++}$, we choose a $\mu_{x}$-induced isometry $\mu_{x}^{+}$such $\mu_{x, 0}^{+} \times \mu_{x, 1}^{+}$is $\mu_{x}^{+}$-induced. In the remaining cases, we choose $\mu_{x}^{+}$as in the comments following (5.8). In all cases, $\mu_{x}^{+}$ is $\mu_{x}$-induced, $\mu_{x, 0}^{+} \times \mu_{x, 1}^{+}$is $\mu_{x}^{+}$-induced, and $\mu_{x}^{+}$is perfect.

Step \%. Let $\Lambda$ run over all splitting mappings of $H_{1}$ such that the $p$-regular and $p^{\prime}$-regular parts $\Lambda^{\prime}$ and $\Lambda^{\prime \prime}$ of $\Lambda$ are splitting mappings of $H_{x, 01}$ and $Y \nmid S\left(\Omega_{x, 1}\right)$. We label the irreducible characters $\psi_{\Lambda, \pm}$ of $H_{1}^{+}$so that $(4 \mathrm{H})$ (ii) holds. The conjugacy class $\mathcal{C}_{\Lambda}$ of $H_{1}$ contains the conjugacy class $\mathcal{C}_{\Lambda^{\prime}} \mathcal{C}_{\Lambda^{\prime \prime}}$ of $H_{x, 01} H_{x, 1}$. If $\left|\Omega_{x, 0}\right| \geq 1$, we label $\mathcal{C}_{\Lambda, \pm}$ so that

$$
\begin{aligned}
& \mathcal{C}_{\Lambda,+}=\left(\mathcal{C}_{\Lambda^{\prime},+} \mathcal{C}_{\Lambda^{\prime \prime},+}\right)^{H_{1}^{+}}=\left(\mathcal{C}_{\Lambda^{\prime},-} \mathcal{C}_{\Lambda^{\prime \prime},-}\right)^{H_{1}^{+}}, \\
& \mathcal{C}_{\Lambda,-}=\left(\mathcal{C}_{\Lambda^{\prime},+} \mathcal{C}_{\Lambda^{\prime \prime},-}\right)^{H_{1}^{+}}=\left(\mathcal{C}_{\Lambda^{\prime},-} \mathcal{C}_{\Lambda^{\prime \prime},+}\right)^{H_{1}^{+}} .
\end{aligned}
$$

If $\left|\Omega_{x, 0}\right|=0$, then $H_{x, 01}=1$, and we label $\mathcal{C}_{\Lambda, \pm}$ so that $\mathcal{C}_{\Lambda,+}=\mathcal{C}_{\Lambda^{\prime \prime},+}$ and $\mathcal{C}_{\Lambda,-}=$ $\mathcal{C}_{\Lambda^{\prime \prime},-}$.

Step 8. Let $\lambda$ run over all self-dual partitions of $|\Pi|$ with $p$-core $\lambda_{0}$ and $p$-quotient $\Lambda$ as in step 7 . Let $\lambda^{\prime}$ and $\lambda^{\prime \prime}$ be the self-dual partitions gotten from $\lambda$ by removing respectively all $(i, i)$-hooks of $p$-singular length and all $(i, i)$-hooks of $p$-regular length. We call $\lambda^{\prime}$ and $\lambda^{\prime \prime}$ the $p$-regular and $p^{\prime}$-regular parts of $\lambda$. In particular, $\lambda^{\prime}$ 
has $p$-core $\lambda_{0}$ and $p$-quotient $\Lambda^{\prime}$, and $\lambda^{\prime \prime}$ has $p$-core $\emptyset$ and $p$-quotient $\Lambda^{\prime \prime}$. If $\left|\lambda^{\prime}\right| \geq 3$, we label the conjugacy classes $\mathcal{C}_{\eta(\lambda), \pm}$ of $G^{+}$so that

$$
\begin{aligned}
& \mathcal{C}_{\eta(\lambda),+}=\left(\mathcal{C}_{\eta\left(\lambda^{\prime}\right),+} \mathcal{C}_{\Lambda^{\prime \prime},+}\right)^{G^{+}}=\left(\mathcal{C}_{\eta\left(\lambda^{\prime}\right),-} \mathcal{C}_{\Lambda^{\prime \prime},-}\right)^{G^{+}}, \\
& \mathcal{C}_{\eta(\lambda),-}=\left(\mathcal{C}_{\eta\left(\lambda^{\prime}\right),+} \mathcal{C}_{\Lambda^{\prime \prime},-}\right)^{G^{+}}=\left(\mathcal{C}_{\eta\left(\lambda^{\prime}\right),-} \mathcal{C}_{\Lambda^{\prime \prime},+}\right)^{G^{+}} .
\end{aligned}
$$

If $\left|\lambda^{\prime}\right| \leq 1$, then $G_{x, 0}=1$, and we label the conjugacy classes $\mathcal{C}_{\eta(\lambda), \pm}$ of $G^{+}$so that $\mathcal{C}_{\eta(\lambda),+}=\mathcal{C}_{\Lambda^{\prime \prime},+}$ and $\mathcal{C}_{\eta(\lambda),-}=\mathcal{C}_{\Lambda^{\prime \prime},-}$. We then label the irreducible characters $\chi_{\lambda, \pm}$ of $G^{+}$so that (4A) holds.

Step 9. The $\lambda$ in step 8 determine splitting classes $\mathcal{C}_{\eta\left(\lambda_{0}\right), \Lambda}=\mathcal{C}_{\eta\left(\lambda_{0}\right)} \mathcal{C}_{\Lambda}$ of $H$. If $\left|\lambda_{0}\right| \geq 3$, we label the classes $\mathcal{C}_{\eta\left(\lambda_{0}\right), \Lambda, \pm}$ of $H^{+}$so that

$$
\begin{aligned}
& \mathcal{C}_{\eta\left(\lambda_{0}\right), \Lambda,+}=\left(\mathcal{C}_{\eta\left(\lambda_{0}\right),+} \mathcal{C}_{\Lambda,+}\right)^{H^{+}}=\left(\mathcal{C}_{\eta\left(\lambda_{0}\right),-} \mathcal{C}_{\Lambda,-}\right)^{H^{+}}, \\
& \mathcal{C}_{\eta\left(\lambda_{0}\right), \Lambda,-}=\left(\mathcal{C}_{\eta\left(\lambda_{0}\right),+} \mathcal{C}_{\Lambda,-}\right)^{H^{+}}=\left(\mathcal{C}_{\eta\left(\lambda_{0}\right),-} \mathcal{C}_{\Lambda,+}\right)^{H^{+}} .
\end{aligned}
$$

We label the irreducible constituents $\left(\chi_{\lambda_{0}} \psi_{\Lambda}\right)_{ \pm}$of $\operatorname{Res}_{H^{+}}^{H}\left(\chi_{\lambda_{0}} \psi_{\Lambda}\right)$ so that

$$
\begin{aligned}
& \operatorname{Res}_{H_{0}^{+} H_{1}^{+}}^{H^{+}}\left(\left(\chi_{\lambda_{0}} \psi_{\Lambda}\right)_{+}\right)=\chi_{\lambda_{0},+} \psi_{\Lambda,+}+\chi_{\lambda_{0},-} \psi_{\Lambda,-}, \\
& \operatorname{Res}_{H_{0}^{+} H_{1}^{+}}^{H^{+}}\left(\left(\chi_{\lambda_{0}} \psi_{\Lambda}\right)_{-}\right)=\chi_{\lambda_{0},+} \psi_{\Lambda,-}+\chi_{\lambda_{0},-} \psi_{\Lambda,+} .
\end{aligned}
$$

If $\left|\lambda_{0}\right| \leq 1$, then $H_{x, 0}=1$, and we label the classes $\mathcal{C}_{\eta\left(\lambda_{0}\right), \Lambda, \pm}$ of $H^{+}$so that $\mathcal{C}_{\eta\left(\lambda_{0}\right), \Lambda,+}=\mathcal{C}_{\Lambda,+}$ and $\mathcal{C}_{\eta\left(\lambda_{0}\right), \Lambda,-}=\mathcal{C}_{\Lambda,-}$. We label the irreducible constituents $\left(\chi_{\lambda_{0}} \psi_{\Lambda}\right)_{ \pm}$of $\operatorname{Res}_{H^{+}}^{H}\left(\chi_{\lambda_{0}} \psi_{\Lambda}\right)$ so that

$$
\left(\chi_{\lambda_{0}} \psi_{\Lambda}\right)_{+}=1_{S\left(\Pi_{0}\right)} \times \psi_{\Lambda,+}, \quad\left(\chi_{\lambda_{0}} \psi_{\Lambda}\right)_{-}=1_{S\left(\Pi_{0}\right)} \times \psi_{\Lambda,-} .
$$

Step 10. Let $\lambda$ run over all self-dual partitions of $|\Pi|$ with $p$-core $\lambda_{0}$ and a $p$-regular $p$-quotient $\Lambda$. We label arbitrarily the irreducible characters $\chi_{\lambda, \pm}$ and the conjugacy classes $\mathcal{C}_{\Lambda, \pm}$ of $G^{+}$. Similarly, we label arbitrarily the irreducible constituents $\left(\chi_{\lambda_{0}} \psi_{\Lambda}\right)_{ \pm}$of $\operatorname{Res}_{H^{+}}^{H}\left(\chi_{\lambda_{0}} \psi_{\Lambda}\right)$ and the conjugacy classes $\mathcal{C}_{\eta\left(\lambda_{0}\right), \Lambda, \pm}=\left(\mathcal{C}_{\eta\left(\lambda_{0}\right)} \mathcal{C}_{\Lambda}\right)_{ \pm}$of $H^{+}$.

Step 11. Let $\lambda$ run over all self-dual partitions of $|\Pi|$ with $p$-core $\lambda_{0}$. Let $\mu=(\mathrm{J}, \epsilon)$. We choose the $\mu$-induced isometry $\mu^{+}=\left(\mathrm{J}^{+}, \epsilon^{+}\right)$so that

$$
\mathrm{J}^{+}\left(\chi_{\lambda, \pm}\right)= \begin{cases}\mathrm{J}\left(\chi_{\lambda}\right)_{ \pm}=\left(\chi_{\lambda_{0}} \psi_{\Lambda}\right)_{ \pm} & \text {if }\left(-\epsilon_{p}\right)^{d_{\lambda}} \sigma_{\lambda}=1, \\ \mathrm{~J}\left(\chi_{\lambda}\right)_{ \pm}=\left(\chi_{\lambda_{0}} \psi_{\Lambda}\right)_{\mp} & \text { if }\left(-\epsilon_{p}\right)^{d_{\lambda}} \sigma_{\lambda}=-1,\end{cases}
$$

where $d_{\lambda}$ is the number of diagonal nodes of $\lambda^{\prime \prime}$ and

$$
\sigma_{\lambda}=\frac{\sqrt{\epsilon_{\lambda^{\prime}}}\left(\sqrt{\epsilon_{p}}\right)^{d_{\lambda}} \sqrt{\epsilon_{\lambda_{p^{*}}}}}{\sqrt{\epsilon_{\lambda}}}
$$

Note that $\sigma_{\lambda}= \pm 1$. Indeed, if $\eta_{i i}\left(\lambda_{p^{*}}\right)=2 u_{i}+1$, then

$$
\begin{aligned}
\epsilon_{\lambda_{p^{*}}} & =(-1)^{\frac{1}{2}\left[\left(\sum\left(2 u_{i}+1\right)\right)-d_{\lambda}\right]} \\
& =(-1)^{\sum u_{i}}
\end{aligned}
$$

and

$$
\begin{aligned}
\epsilon_{\lambda^{\prime \prime}} & =(-1)^{\frac{1}{2}\left[\left(\sum\left(2 u_{i} p+p\right)\right)-d_{\lambda}\right]} \\
& =(-1)^{\left(\sum u_{i} p\right)+d_{\lambda}\left(\frac{p-1}{2}\right)} .
\end{aligned}
$$


Since $\sum u_{i} p \equiv \sum u_{i}(\bmod 2)$ and $\epsilon_{p}=(-1)^{\left(\frac{p-1}{2}\right)}$, it follows that $\epsilon_{\lambda^{\prime \prime}}=\left(\epsilon_{p}\right)^{d_{\lambda}} \epsilon_{\lambda_{p^{*}}}$ and $\epsilon_{\lambda}=\epsilon_{\lambda^{\prime}}\left(\epsilon_{p}\right)^{d_{\lambda}} \epsilon_{\lambda_{p^{*}}}$. Thus $\sigma_{\lambda}= \pm 1$.

Proposition (5C). Let $\lambda$ be a self-dual partition of $n$ with $p$-core $\lambda_{0}$ and p-quotient $\Lambda$, and let $x \in\left\{x_{1}, x_{2}, \ldots, x_{w}\right\}$. Then

$$
\mathrm{R}_{\mu_{x}^{+}, p^{\prime}}\left(d_{G^{+}}^{x}\left(\chi_{\lambda,+}-\chi_{\lambda,-}\right)\right)(y)=0
$$

unless $\left|\lambda_{p^{*}}\right|=\left|\Omega_{x, 1}\right|$ and $y \in\left(H_{x, 00}^{+} H_{x, 01}^{+} H_{x, 1}^{+}\right)_{p^{\prime}}$. Moreover, if $\left|\lambda_{p^{*}}\right|=\left|\Omega_{x, 1}\right|$ and $y=y_{00} y_{01} y_{1}$ with $y_{00} \in\left(H_{x, 00}^{+}\right)_{p^{\prime}}, y_{01} \in\left(H_{x, 01}^{+}\right)_{p^{\prime}}, y_{1} \in\left(H_{x, 1}^{+}\right)_{p^{\prime}}$, then

$$
\begin{aligned}
\mathrm{R}_{\mu_{x}^{+}, p^{\prime}}\left(d_{G^{+}}^{x}\left(\chi_{\lambda,+}-\chi_{\lambda,-}\right)\right)(y)= & \sigma_{\lambda} \epsilon_{x, 0}\left(\chi_{\lambda^{\prime}}\right)\left(\chi_{\lambda_{0},+}-\chi_{\lambda_{0},-}\right)\left(y_{00}\right) \\
& \times\left(\psi_{\Lambda^{\prime},+}-\psi_{\Lambda^{\prime},-}\right)\left(y_{01}\right)\left(\psi_{\Lambda^{\prime \prime},+}-\psi_{\Lambda^{\prime \prime},-}\right)\left(x y_{1}\right) .
\end{aligned}
$$

Here $\lambda^{\prime}$ is the p-regular part of $\lambda ; \psi_{\Lambda^{\prime}}$ and $\psi_{\Lambda^{\prime \prime}}$ are the irreducible characters of $Y \nmid S\left(\Omega_{x, 0}\right)$ and $Y \nmid S\left(\Omega_{x, 1}\right)$ parametrized by the p-regular and p-singular parts $\Lambda^{\prime}$ and $\Lambda^{\prime \prime}$ of $\Lambda$; and the factor $\left(\chi_{\lambda_{0},+}-\chi_{\lambda_{0},-}\right)$ or $\left(\psi_{\Lambda^{\prime},+}-\psi_{\Lambda^{\prime},-}\right)$ is interpreted as 1 when $\left|\lambda_{0}\right| \leq 1$ or $\left|\Omega_{x, 0}\right|=0$.

Proof. Let $\rho_{\Lambda^{\prime \prime}, \pm}=\operatorname{Res}_{\left(P 2 S\left(\Omega_{x, 1}\right)\right)^{+}}^{\left(Y 2\left(\Omega_{x, 1}\right)\right)^{+}}\left(\psi_{\Lambda^{\prime \prime}, \pm}\right)$ if $\left|\lambda_{p^{*}}\right|=\left|\Omega_{x, 1}\right|$, and let $\rho_{\Lambda^{\prime \prime}, \pm}$ be the zero function if $\left|\lambda_{p^{*}}\right| \neq\left|\Omega_{x, 1}\right|$. We show first that

$$
d_{G^{+}}^{x}\left(\chi_{\lambda,+}-\chi_{\lambda,-}\right)=\sigma_{\lambda} \operatorname{Ind}_{G_{x}^{++}}^{G_{x}^{+}}\left(d_{G_{x, 0}^{+}}^{1}\left(\chi_{\lambda^{\prime},+}\right) \times d_{G_{x, 1}^{+}}^{x}\left(\rho_{\Lambda^{\prime \prime},+}-\rho_{\Lambda^{\prime \prime},-}\right)\right),
$$

where $d_{G_{x, 0}^{+}}^{1}\left(\chi_{\lambda^{\prime},+}\right)$ is interpreted as 1 when $\left|\lambda^{\prime}\right| \leq 1$. Indeed, let $z_{0} z_{1} \in G_{x, p^{\prime}}^{+}$, where $z_{0} \in G_{x, 0}$ and $z_{1} \in G_{x, 1}$. By $(4 \mathrm{~A}),\left(\chi_{\lambda,+}-\chi_{\lambda,-}\right)\left(x z_{0} z_{1}\right) \neq 0$ only if $x z_{0} z_{1} \in \mathcal{C}_{\eta(\lambda)}$, or equivalently, only if $\left|\lambda_{p^{*}}\right|=\left|\Omega_{x, 1}\right|, z_{0}$ is in the class $\mathcal{C}_{\eta\left(\lambda^{\prime}\right)}$ of $G_{x, 0}$, and $x z_{1}$ is in the class $\mathcal{C}_{\Lambda^{\prime \prime}}$ of $G_{x, 1}$. Thus (5.9) holds if $\left|\lambda_{p^{*}}\right| \neq\left|\Omega_{x, 1}\right|$, since both sides of (5.9) are zero. Suppose $\left|\lambda_{p^{*}}\right|=\left|\Omega_{x, 1}\right|$. Then

$$
d_{G^{+}}^{x}\left(\chi_{\lambda,+}-\chi_{\lambda,-}\right)\left(z_{0} z_{1}\right)= \begin{cases} \pm \sqrt{\epsilon_{\lambda} \eta(\lambda)} & \text { if } x z_{0} z_{1} \in \mathcal{C}_{\eta(\lambda), \pm} \\ 0 & \text { if } x z_{0} z_{1} \notin \mathcal{C}_{\eta(\lambda), \pm}\end{cases}
$$

On the other hand, $\operatorname{Ind}_{G_{x}^{++}}^{G_{x}^{+}}\left(d_{G_{x, 0}^{+}}^{1}\left(\chi_{\lambda^{\prime},+}\right) \times d_{G_{x, 1}^{+}}^{x}\left(\rho_{\Lambda^{\prime \prime},+}-\rho_{\Lambda^{\prime \prime},-}\right)\right)$ has support on $G_{x}^{++}$, where it is equal to

$$
d_{G_{x, 0}^{+}}^{1}\left(\chi_{\lambda^{\prime},+}-\chi_{\lambda^{\prime},-}\right) \times d_{G_{x, 1}^{+}}^{x}\left(\rho_{\Lambda^{\prime \prime},+}-\rho_{\Lambda^{\prime \prime},-}\right) .
$$

Moreover, $(4 \mathrm{~A})$ and $(4 \mathrm{~F})$ imply that $\left(\chi_{\lambda^{\prime},+}-\chi_{\lambda^{\prime},-}\right)\left(z_{0}\right)$ and $\left(\psi_{\Lambda^{\prime \prime},+}-\psi_{\Lambda^{\prime \prime},-}\right)\left(x z_{1}\right)$ are zero except for

$$
\begin{array}{cl}
\left(\chi_{\lambda^{\prime},+}-\chi_{\lambda^{\prime},-}\right)\left(z_{0}\right)= \pm \sqrt{\epsilon_{\lambda^{\prime}} \eta\left(\lambda^{\prime}\right)} & \text { if } z_{0} \in \mathcal{C}_{\eta\left(\lambda^{\prime}\right), \pm}, \\
\left(\psi_{\Lambda^{\prime \prime},+}-\psi_{\Lambda^{\prime \prime},-}\right)\left(x z_{1}\right)= \pm\left(\sqrt{\epsilon_{p} p}\right)^{d_{\lambda}} \sqrt{\epsilon_{\lambda_{p^{*}}} \eta\left(\lambda_{p^{*}}\right)} & \text { if } x z_{1} \in \mathcal{C}_{\Lambda^{\prime \prime}, \pm} .
\end{array}
$$

Here $\left(\chi_{\lambda^{\prime},+}-\chi_{\lambda^{\prime},-}\right)\left(z_{0}\right)$ and $\pm \sqrt{\epsilon_{\lambda^{\prime}} \eta\left(\lambda^{\prime}\right)}$ are interpreted as 1 when $\left|\lambda^{\prime}\right| \leq 1$. Since

$$
\epsilon_{\lambda}=\epsilon_{\lambda^{\prime}}\left(\epsilon_{p}\right)^{d_{\lambda}} \epsilon_{\lambda_{p^{*}}}, \quad \sqrt{\eta(\lambda)}=\sqrt{\eta\left(\lambda^{\prime}\right) p^{d_{\lambda}} \eta\left(\lambda_{p^{*}}\right)},
$$

(5.9) follows from the labeling in step 8. 
Suppose $\left|\lambda^{\prime}\right| \geq 3$. Then

$$
\begin{aligned}
& \mathrm{R}_{\mu_{x}^{+}, p^{\prime}}\left(d_{G^{+}}^{x}\left(\chi_{\lambda,+}-\chi_{\lambda,-}\right)\right) \\
& =\mathrm{R}_{\mu_{x}^{+}, p^{\prime}}\left(\sigma_{\lambda} \operatorname{Ind}_{G_{x}^{++}}^{G_{x}^{+}}\left(d_{G_{x, 0}^{+}}^{1}\left(\chi_{\lambda^{\prime},+}\right) \times d_{G_{x, 1}^{+}}^{x}\left(\rho_{\Lambda^{\prime \prime},+}-\rho_{\Lambda^{\prime \prime},-}\right)\right)\right) \\
& =\sigma_{\lambda} \operatorname{Ind}_{H_{x}^{++}}^{H_{x}^{+}}\left(\mathrm{R}_{\mu_{x, 0}^{+} \times \mu_{x, 1}^{+}, p^{\prime}}\left(d_{G_{x, 0}^{+}}^{1}\left(\chi_{\lambda^{\prime},+}\right) \times d_{G_{x, 1}^{+}}^{x}\left(\rho_{\Lambda^{\prime \prime},+}-\rho_{\Lambda^{\prime \prime},-}\right)\right)\right) \\
& \text { (since } \mu_{x}^{+} \text {induces } \mu_{x, 0}^{+} \times \mu_{x, 1}^{+} \text {) } \\
& =\sigma_{\lambda} \operatorname{Ind}_{H_{x}^{++}}^{H_{x}^{+}}\left(\mathrm{R}_{\mu_{x, 0, p^{\prime}}^{+}}\left(d_{G_{x, 0}^{+}}^{1}\left(\chi_{\lambda^{\prime},+}\right)\right) \times \mathrm{R}_{\mu_{x, 1, p^{\prime}}^{+}}\left(d_{G_{x, 1}^{+}}^{x}\left(\rho_{\Lambda^{\prime \prime},+}-\rho_{\Lambda^{\prime \prime},-}\right)\right)\right) \\
& =\sigma_{\lambda} \operatorname{Ind}_{H_{x}^{++}}^{H_{x}^{+}}\left(d_{H_{x, 0}^{+}}^{1}\left(\mathrm{R}_{\mu_{x, 0}^{+}}\left(\chi_{\lambda^{\prime},+}\right)\right) \times d_{H_{x, 1}^{+}}^{x}\left(\mathrm{R}_{\mu_{x, 1}^{+}}\left(\rho_{\Lambda^{\prime \prime},+}-\rho_{\Lambda^{\prime \prime},-}\right)\right)\right) \\
& \text { (since } \mu_{x, 0}^{+} \text {is perfect by }(5 \mathrm{~B}) \text { and } \mu_{x, 1}^{+} \text {is the identity isometry) } \\
& =\sigma_{\lambda} d_{H_{x}^{+}}^{x}\left(\operatorname{Ind}_{H_{x}^{++}}^{H_{x}^{+}}\left(\mathrm{R}_{\mu_{x, 0}^{+} \times \mu_{x, 1}^{+}}\left(\chi_{\lambda^{\prime},+} \times\left(\rho_{\Lambda^{\prime \prime},+}-\rho_{\Lambda^{\prime \prime},-}\right)\right)\right)\right) \text {. }
\end{aligned}
$$

Thus $\mathrm{R}_{\mu_{x}^{+}, p^{\prime}}\left(d_{G^{+}}^{x}\left(\chi_{\lambda,+}-\chi_{\lambda,-}\right)\right)$ has support on $\left(H_{x}^{++}\right)_{p^{\prime}}$, where it is equal to

$$
\sigma_{\lambda} d_{H_{x}^{+}}^{x}\left(\mathrm{R}_{\mu_{x, 0}^{+}}\left(\chi_{\lambda^{\prime},+}-\chi_{\lambda^{\prime},-}\right) \times \mathrm{R}_{\mu_{x, 1}^{+}}\left(\rho_{\Lambda^{\prime \prime},+}-\rho_{\Lambda^{\prime \prime},-}\right)\right) .
$$

In turn, $\mathrm{R}_{\mu_{x, 0}^{+}}\left(\chi_{\lambda^{\prime},+}-\chi_{\lambda^{\prime},-}\right)$ has support on $H_{x, 00}^{+} H_{x, 01}^{+}$, where it is equal to

$$
\epsilon_{x, 0}\left(\chi_{\lambda^{\prime}}\right)\left(\chi_{\lambda_{0},+}-\chi_{\lambda_{0},-}\right) \times\left(\psi_{\Lambda^{\prime},+}-\psi_{\Lambda^{\prime},-}\right) .
$$

Thus $\mathrm{R}_{\mu_{x}^{+}, p^{\prime}}\left(d_{G^{+}}^{x}\left(\chi_{\lambda,+}-\chi_{\lambda,-}\right)\right)$ has support on $\left(H_{x, 00}^{+} H_{x, 01}^{+} H_{x, 1}^{+}\right)_{p^{\prime}}$, and the first half of (5C) holds. The argument shows that

$$
\begin{aligned}
\mathrm{R}_{\mu_{x}^{+}, p^{\prime}}\left(d_{G^{+}}^{x}\left(\chi_{\lambda,+}-\chi_{\lambda,-}\right)\right)(y)= & \sigma_{\lambda} \epsilon_{x, 0}\left(\chi_{\lambda^{\prime}}\right)\left(\chi_{\lambda_{0},+}-\chi_{\lambda_{0},-}\right)\left(y_{00}\right) \\
& \times\left(\psi_{\Lambda^{\prime},+}-\psi_{\Lambda^{\prime},-}\right)\left(y_{01}\right)\left(\psi_{\Lambda^{\prime \prime},+}-\psi_{\Lambda^{\prime \prime},-}\right)\left(x y_{1}\right)
\end{aligned}
$$

if $\left|\lambda_{p^{*}}\right|=\left|\Omega_{x, 1}\right|$ and $y=y_{00} y_{01} y_{1}$ with $y_{00} \in\left(H_{x, 00}^{+}\right)_{p^{\prime}}, y_{01} \in\left(H_{x, 01}^{+}\right)_{p^{\prime}}$, and $y_{1} \in\left(H_{x, 1}^{+}\right)_{p^{\prime}}$. Thus (5C) holds when $\left|\lambda^{\prime}\right| \geq 3$.

Suppose $\left|\lambda^{\prime}\right| \leq 1$. Then $G_{x}^{+}=G_{x}^{++}$, and (5.11) reduces to

$$
\begin{aligned}
\mathrm{R}_{\mu_{x}^{+}, p^{\prime}}\left(d_{G^{+}}^{x}\left(\chi_{\lambda,+}-\chi_{\lambda,-}\right)\right) & =\mathrm{R}_{\mu_{x}^{+}, p^{\prime}}\left(\sigma_{\lambda} d_{G_{x, 1}^{+}}^{x}\left(\rho_{\Lambda^{\prime \prime},+}-\rho_{\Lambda^{\prime \prime},-}\right)\right) \\
& =\sigma_{\lambda} \mathrm{R}_{\mu_{x, 1}^{+}, p^{\prime}}\left(d_{G_{x, 1}^{+}}^{x}\left(\rho_{\Lambda^{\prime \prime},+}-\rho_{\Lambda^{\prime \prime},-}\right)\right) \\
& =\sigma_{\lambda} d_{H_{x, 1}^{+}}^{x}\left(\mathrm{R}_{\mu_{x, 1}^{+}}\left(\rho_{\Lambda^{\prime \prime},+}-\rho_{\Lambda^{\prime \prime},-}\right)\right) .
\end{aligned}
$$

Then (5C) holds trivially in this case, since $\left(H_{x, 00}^{+} H_{x, 01}^{+} H_{x, 1}^{+}\right)_{p^{\prime}}=\left(H_{x, 1}^{+}\right)_{p^{\prime}}$,

Proposition (5D). Let $\lambda$ be a self-dual partition of $n$ with $p$-core $\lambda_{0}$ and $p$-quotient $\Lambda$, and let $x \in\left\{x_{1}, x_{2}, \ldots, x_{w}\right\}$. Then

$$
d_{H^{+}}^{x}\left(\mathrm{R}_{\mu^{+}}\left(\left(\chi_{\lambda,+}-\chi_{\lambda,-}\right)\right)(y)=0\right.
$$

unless $\left|\lambda_{p^{*}}\right|=\left|\Omega_{x, 1}\right|$ and $y \in\left(H_{x, 00}^{+} H_{x, 01}^{+} H_{x, 1}^{+}\right)_{p^{\prime}}$. Moreover, if $\left|\lambda_{p^{*}}\right|=\left|\Omega_{x, 1}\right|$ and $y=y_{00} y_{01} y_{1}$ with $y_{00} \in\left(H_{x, 00}^{+}\right)_{p^{\prime}}, y_{01} \in\left(H_{x, 01}^{+}\right)_{p^{\prime}}, y_{1} \in\left(H_{x, 1}^{+}\right)_{p^{\prime}}$, then

$$
\begin{aligned}
d_{H^{+}}^{x}\left(\mathrm{R}_{\mu^{+}}\left(\chi_{\lambda,+}-\chi_{\lambda,-}\right)\right)(y)= & \sigma_{\lambda}\left(-\epsilon_{p}\right)^{d_{\lambda}} \epsilon\left(\chi_{\lambda}\right)\left(\chi_{\lambda_{0},+}-\chi_{\lambda_{0},-}\right)\left(y_{00}\right) \\
& \times\left(\psi_{\Lambda^{\prime},+}-\psi_{\Lambda^{\prime},-}\right)\left(y_{01}\right)\left(\psi_{\Lambda^{\prime \prime},+}-\psi_{\Lambda^{\prime \prime},-}\right)\left(x y_{1}\right) .
\end{aligned}
$$


Here $\psi_{\Lambda^{\prime}}$ and $\psi_{\Lambda^{\prime \prime}}$ are the irreducible characters of $Y \backslash S\left(\Omega_{x, 0}\right)$ and $Y \backslash S\left(\Omega_{x, 0}\right)$ parametrized by the p-regular and p-singular parts $\Lambda^{\prime}$ and $\Lambda^{\prime \prime}$ of $\Lambda$, and the factor $\left(\chi_{\lambda_{0},+}-\chi_{\lambda_{0},-}\right)$ or $\left(\psi_{\Lambda^{\prime},+}-\psi_{\Lambda^{\prime},-}\right)$ is interpreted as 1 when $\left|\lambda_{0}\right| \leq 1$ or $\left|\Omega_{x, 0}\right|=0$.

Proof. For $y \in\left(H_{x}^{+}\right)_{p^{\prime}}$ we have

$$
\begin{aligned}
d_{H^{+}}^{x}\left(\mathrm{R}_{\mu^{+}}\left(\chi_{\lambda,+}-\chi_{\lambda,-}\right)\right)(y) & =\mathrm{R}_{\mu^{+}}\left(\chi_{\lambda,+}-\chi_{\lambda,-}\right)(x y) \\
& =\left(-\epsilon_{p}\right)^{d_{\lambda}} \sigma_{\lambda} \epsilon\left(\chi_{\lambda}\right)\left(\mathrm{J}^{+}\left(\chi_{\lambda,+}\right)-\mathrm{J}^{+}\left(\chi_{\lambda,-}\right)\right)(x y) .
\end{aligned}
$$

Let $y=y_{00} y_{01} y_{1}$, where $y_{00} \in\left(H_{x, 00}\right)_{p^{\prime}}, y_{01} \in\left(H_{x, 01}\right)_{p^{\prime}}, y_{1} \in\left(H_{x, 1}\right)_{p^{\prime}}$. Thus $y_{00} \in\left(H_{0}\right)_{p^{\prime}}, y_{01} y_{1} \in\left(H_{1}\right)_{p^{\prime}}$, and

$$
\begin{aligned}
& \left(\mathrm{J}^{+}\left(\chi_{\lambda,+}\right)-\mathrm{J}^{+}\left(\chi_{\lambda,-}\right)\right)(x y) \\
& \quad= \begin{cases}\left(\chi_{\lambda_{0},+}-\chi_{\lambda_{0},-}\right)\left(y_{00}\right)\left(\psi_{\Lambda,+}-\psi_{\Lambda,-}\right)\left(x y_{01} y_{1}\right) & \text { if } x y \in H_{0}^{+} H_{1}^{+}, \\
0 & \text { if } x y \notin H_{0}^{+} H_{1}^{+}\end{cases}
\end{aligned}
$$

by the labeling of $\mathrm{J}^{+}\left(\chi_{\lambda, \pm}\right)$ in steps $8-10$. Suppose $d_{H^{+}}^{x}\left(\mathrm{R}_{\mu^{+}}\left(\chi_{\lambda,+}-\chi_{\lambda,-}\right)\right)(y) \neq 0$. Then $y_{00} \in\left(H_{x, 00}^{+}\right)_{p^{\prime}}, y_{01} y_{1} \in\left(H_{1}^{+}\right)_{p^{\prime}}$, and $\left(\psi_{\Lambda,+}-\psi_{\Lambda,-}\right)\left(x y_{01} y_{1}\right) \neq 0$. Let $H_{1}^{\prime}$ and $H_{1}^{\prime \prime}$ be the subgroups of $H_{1}$ associated to $\Lambda^{\prime}$ and $\Lambda^{\prime \prime}$. By (4H) (i) there exist elements $u^{\prime}$ in a $p$-regular splitting class of $H_{1}^{\prime}, u^{\prime \prime}$ in a $p$-singular splitting class of $H_{1}^{\prime \prime}$, and $h \in H_{1}^{+}$such that $\left(x y_{01} y_{1}\right)^{h}=u^{\prime} u^{\prime \prime}$. A comparison of the $p$-regular and $p$-singular cycle types of these elements as elements of $S(\Gamma \times \Omega)$ shows that $\left(y_{01}\right)^{h}=u^{\prime},\left(x y_{1}\right)^{h}=u^{\prime \prime}$. Thus $\left|\lambda_{p^{*}}\right|=\left|\Omega_{x, 1}\right|, y_{01} \in H_{x, 01}^{+}, y_{1} \in H_{x, 1}^{+}$, and the first part of (5D) holds. Now suppose $\left|\lambda_{p^{*}}\right|=\left|\Omega_{x, 1}\right|, y_{00} \in\left(H_{x, 00}^{+}\right)_{p^{\prime}}, y_{01} \in\left(H_{x, 01}^{+}\right)_{p^{\prime}}$, and $y_{1} \in\left(H_{x, 1}^{+}\right)_{p^{\prime}}$. We can then choose $Y 2 S\left(\Omega_{x, 0}\right)$ and $Y 2 S\left(\Omega_{x, 1}\right)$ as the subgroups $H_{1}^{\prime}$ and $H_{1}^{\prime \prime}$ of $H_{1}$ associated to $\Lambda^{\prime}$ and $\Lambda^{\prime \prime}$. The second part of (5D) now follows from $(4 \mathrm{H})(\mathrm{ii})$, the labelings in steps $8-9$, and the definition of $J^{+}\left(\chi_{\lambda, \pm}\right)$.

Proposition (5E). The diagram

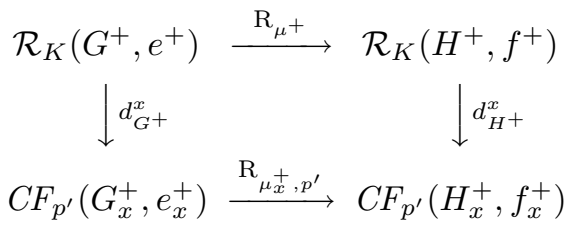

is commutative for all $x$ in $\left\{x_{1}, x_{2}, \ldots, x_{w}\right\}$.

Proof. Suppose $\chi^{+}=\operatorname{Res}_{G}^{G}(\chi)$ for $\chi \in(G, e)^{\vee}$. Then

$$
d_{H^{+}}^{x}\left(\mathrm{R}_{\mu^{+}}\left(\chi^{+}\right)\right)=\mathrm{R}_{\mu_{x}^{+}, p^{\prime}}\left(d_{G^{+}}^{x}\left(\chi^{+}\right)\right),
$$

that is, (5.12) is commutative for such $\chi^{+}$. Indeed, $d_{H}^{x} \circ \mathrm{R}_{\mu}=\mathrm{R}_{\mu_{x}, p^{\prime}} \circ d_{G}^{x}$ by (3B), and

$$
\mathrm{R}_{\mu^{+}} \circ \operatorname{Res}_{G^{+}}^{G}=\operatorname{Res}_{H^{+}}^{H} \circ \mathrm{R}_{\mu}, \quad \mathrm{R}_{\mu_{x}^{+}} \circ \operatorname{Res}_{G_{x}^{+}}^{G_{x}}=\operatorname{Res}_{H_{x}^{+}}^{H_{x}} \circ \mathrm{R}_{\mu_{x}}
$$


since $\mu^{+}$is $\mu$-induced and $\mu_{x}^{+}$is $\mu_{x}$-induced. Thus

$$
\begin{aligned}
d_{H^{+}}^{x} \circ \mathrm{R}_{\mu^{+}} \circ \operatorname{Res}_{G^{+}}^{G} & =d_{H^{+}}^{x} \circ \operatorname{Res}_{H^{+}}^{H} \circ \mathrm{R}_{\mu} \\
& =\operatorname{Res}_{H_{x, p^{\prime}}^{+}}^{H_{x, p^{\prime}}} \circ d_{H}^{x} \circ \mathrm{R}_{\mu} \\
& =\operatorname{Res}_{H_{x, p^{\prime}}^{+}}^{H_{x, p^{\prime}}} \circ \mathrm{R}_{\mu_{x}, p^{\prime}} \circ d_{G}^{x} \\
& =\mathrm{R}_{\mu_{x}^{+}, p^{\prime}} \circ \operatorname{Res}_{G_{x, p^{\prime}}^{+}}^{G_{x}} \circ d_{G}^{x} \\
& =\mathrm{R}_{\mu_{x}^{+}, p^{\prime}} \circ d_{G^{+}}^{x} \circ \operatorname{Res}_{G^{+}}^{G},
\end{aligned}
$$

since $\mu_{x}$ and $\mu_{x}^{+}$are perfect isometries.

Suppose $\chi^{+}=\chi_{\lambda,+}-\chi_{\lambda,-}$, where $\chi_{\lambda} \in(G, e)^{\vee}, \lambda$ is self-dual, and $G_{x}^{+}>G_{x}^{++}$. The commutativity of (5.12) for $\chi^{+}$follows from the expressions for

$$
\mathrm{R}_{\mu_{x}^{+}, p^{\prime}}\left(d_{G^{+}}^{x}\left(\chi_{\lambda,+}-\chi_{\lambda,-}\right)\right)(y), \quad d_{H^{+}}^{x}\left(\mathrm{R}_{\mu^{+}}\left(\chi_{\lambda,+}-\chi_{\lambda,-}\right)\right)(y)
$$

given in $(5 \mathrm{C})$ and $(5 \mathrm{D})$. Indeed, we may suppose that $\Lambda^{\prime}$ is a $p$-regular splitting mapping of $Y<S\left(\Omega_{x, 0}\right)$ and $\Lambda^{\prime \prime}$ is a $p$-singular splitting mapping of $Y \imath S\left(\Omega_{x, 1}\right)$. It remains to show that

$$
\sigma_{\lambda} \epsilon_{x, 0}\left(\chi_{\lambda^{\prime}}\right)=\left(-\epsilon_{p}\right)^{d_{\lambda}} \sigma_{\lambda} \epsilon\left(\chi_{\lambda}\right)
$$

But $\epsilon\left(\chi_{\lambda}\right)=v(\lambda)(-1)^{\left|\lambda_{p^{*}}\right|}=(-1)^{d_{\lambda}}$ since $\lambda$ is self-dual, $\epsilon_{x, 0}\left(\chi_{\lambda^{\prime}}\right)=v\left(\lambda^{\prime}\right)$ since $\lambda^{\prime}$ is $p$-regular, and $v(\lambda) v\left(\lambda^{\prime}\right)=\left(\epsilon_{p}\right)^{d_{\lambda}}$. Thus (5.13) holds.

Suppose $\chi^{+}=\chi_{\lambda,+}-\chi_{\lambda,-}$, where $\chi_{\lambda} \in(G, e)^{\vee}, \lambda$ is self-dual, and $G_{x}^{+}=G_{x}^{++}$. Then $\left|\Pi_{0} \cup\left(\Gamma \times \Omega_{x, 0}\right)\right| \leq 1$ or $\left|\Omega_{x, 1}\right|=1$. If $\left|\Pi_{0} \cup\left(\Gamma \times \Omega_{x, 0}\right)\right| \leq 1$, then $G_{x}^{+}=H_{x}^{+}$, $\mu_{x}^{+}$is the identity isometry, and (5.12) holds trivially. If $\left|\Omega_{x, 1}\right|=1$, then

$$
G_{x}^{+}=A\left(\Pi_{0} \cup\left(\Gamma \times \Omega_{x, 0}\right) \times P, \quad H_{x}^{+}=\left(S\left(\Pi_{0}\right) \times\left(Y \imath S\left(\Omega_{x, 0}\right)\right)\right)^{+} \times P\right.
$$

The previous arguments apply if $\left(\psi_{\Lambda^{\prime \prime},+}-\psi_{\Lambda^{\prime \prime},-}\right)\left(y_{1}\right)$ and $\left(\rho_{\Lambda^{\prime \prime},+}-\rho_{\Lambda^{\prime \prime},-}\right)\left(y_{1}\right)$ are interpreted as 1 when $\left|\lambda_{0}\right| \leq 1$ or $\left|\Omega_{x, 0}\right|=0$. This completes the proof of $(5 \mathrm{E})$.

Theorem $(\mathbf{5 F}) . e^{+}$and $f^{+}$are isotypic blocks of $\mathcal{O G}^{+}$and $\mathrm{OH}^{+}$with local system given by the translates of the $\mu_{x}^{+}$for $x$ in $\left\{x_{1}, x_{2}, \ldots, x_{w}\right\}$.

Proof. The inclusion of $D$ in $G^{+}$and $H^{+}$induces an equivalence of $\mathbf{B r}_{e^{+}, D}\left(G^{+}\right)$ and $\mathbf{B r}_{f^{+}, D}\left(H^{+}\right)$by $[1,(4.1)]$. We claim the diagram (5.12) with $x$ replaced by any generator of $\langle x\rangle$ is a commutative diagram. Indeed, the horizontal arrows in (5.12) depend only on $\langle x\rangle$ and not on $x$. The proofs remain valid when $x$ is replaced by any generator of $\langle x\rangle$. Since $x \in\left\{x_{1}, x_{2}, \ldots, x_{w}\right\}$ and the $w$ subgroups $\left\langle x_{j}\right\rangle$ form a set of representatives for the $N_{G^{+}}\left(D, e_{D}^{+}\right)$-orbits of non-trivial cyclic subgroups of $D,(5 \mathrm{~F})$ holds by transport of structure, as remarked in $\S 1$.

\section{Compatibility}

The Broué conjecture for the principal block holds for all finite groups if it holds for all finite simple non-abelian groups $X$ and if the isotypies for $X$ satisfy compatibility conditions with regard to extensions of $X$ by $p^{\prime}$-groups of automorphisms (see $[10,(5 \mathrm{E})])$. We show that the isotypies constructed in $\S 5$ for alternating groups satisfy these compatibility conditions. We suppose $p>2$ throughout this section.

Let $G$ and $G^{+}$be the symmetric and the alternating groups of degree $n$, let $e$ and $e^{+}$be the 1-blocks of $\mathcal{O} G$ and $\mathcal{O} G^{+}$, and let $e$ and $e^{+}$have the common non-trivial abelian defect group $D$. Moreover, let $H=N_{G}(D)$ and $H^{+}=N_{G^{+}}(D)$, and let $f$ 
and $f^{+}$be the Brauer correspondents of $e$ and $e^{+}$. In particular, $e^{+}$and $f^{+}$are stable under $G$ and $H$. On the other hand, $e=e^{*}$ and $f=f^{*}$ hold if and only if $\left|\Pi_{0}\right| \leq 1$. The maximal subpairs $\left(D, e_{D}\right),\left(D, f_{D}\right),\left(D, e_{D}^{+}\right),\left(D, f_{D}^{+}\right)$for $e, f, e^{+}$, $f^{+}$are unique since $e, f, e^{+}, f^{+}$are the 1-blocks. Let $\left\{\mu_{P}\right\}$ and $\left\{\mu_{P}^{+}\right\}$be the local systems for the isotypies between $e$ and $f$ and between $e^{+}$and $f^{+}$constructed in $\S 3$ and $\S 5$.

Let $\tilde{G}=\operatorname{Aut}\left(G^{+}\right)$, so that $\tilde{G} \geq G$ and $|\tilde{G} / G|=1$ or 2 according as $n \neq 6$ or $n=6$. Let $\tilde{H}=N_{\tilde{G}}(D)$. The inclusion of $\tilde{H}$ in $\tilde{G}$ induces an isomorphism $\alpha: \tilde{G} / G^{+} \rightarrow \tilde{H} / H^{+}$, since $\tilde{G}=\tilde{H} G^{+}$. The compatibility conditions from $[10, \S 3]$ are of three types. The first type $(\tilde{\mathbf{F}} \mathbf{1}),\left(\tilde{\mathbf{F}} \mathbf{2}^{\prime}\right),(\tilde{\mathbf{F}} \mathbf{3})$ relate to fusion:

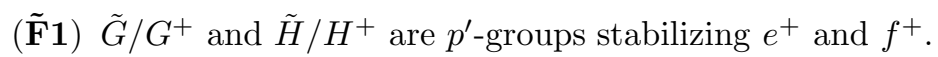

$\left(\tilde{\mathbf{F}} \mathbf{2}^{\prime}\right)$ Given $x$ in $N_{\tilde{G}}(D)$, there exists $y$ in $N_{\tilde{H}}(D)$ with $\alpha\left(x G^{+}\right)=y H^{+}$such that $x$ and $y$ induce the same mapping on $D$. Similarly, given $y$ in $N_{\tilde{H}}(D)$, there exists $x$ in $N_{\tilde{G}}(D)$ with $\alpha\left(x G^{+}\right)=y H^{+}$such that $x$ and $y$ induce the same mapping on $D$.

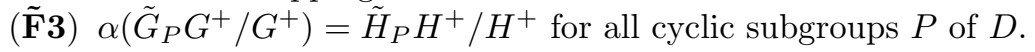

$(\tilde{\mathbf{F}} \mathbf{1})$ holds since $e^{+}$and $f^{+}$are the 1-blocks. $\left(\tilde{\mathbf{F}} \mathbf{2}^{\prime}\right)$ and $(\tilde{\mathbf{F}} \mathbf{3})$ follow easily from $\tilde{G}=\tilde{H} G^{+}$and the structure of $\tilde{G}_{P}$ and $\tilde{H}_{P}$.

The second type $(\tilde{\mathbf{I}} \mathbf{1})$ imposes extendibility conditions on the isometries $\mu_{P}^{+}=$ $\left(J_{P}^{+}, \epsilon_{P}^{+}\right)$for all cyclic $P \leq D$. Namely, let $\alpha_{P}: \tilde{G}_{P} / G_{P}^{+} \longrightarrow \tilde{H}_{P} / H_{P}^{+}$be the isomorphism defined by $\alpha_{P}\left(x G_{P}^{+}\right)=\alpha\left(x G^{+}\right) \cap \tilde{H}_{P}$ for $x \in \tilde{G}_{P}$, and set

$$
\begin{aligned}
& \Delta\left(\alpha_{P}\right)=\left\{(x, y): x \in \tilde{G}_{P}, y \in \tilde{H}_{P}, \alpha_{P}\left(x G_{P}^{+}\right)=y H_{P}^{+}\right\}, \\
& \Delta\left(\mu_{P}^{+}\right)=\left\{\chi_{P}^{+} \times \bar{\psi}_{P}^{+}: \chi_{P}^{+} \in\left(G_{P}^{+}, e_{P}^{+}\right)^{\vee}, \psi_{P}^{+} \in\left(H_{P}^{+}, f_{P}^{+}\right)^{\vee}, \mathrm{J}_{P}^{+}\left(\chi_{P}^{+}\right)=\psi_{P}^{+}\right\} .
\end{aligned}
$$

In particular, $\Delta\left(\alpha_{P}\right)=\Delta(\alpha) \cap\left(\tilde{G}_{P} \times \tilde{H}_{P}\right)$. Then the following hold:

$\left(\tilde{\mathbf{I}}\right.$ 1.1) $\mu_{P}^{+}$is stable under $\Delta\left(\alpha_{P}\right)$.

$(\tilde{\mathbf{I}} 1.2)$ Each $\chi_{P}^{+} \times \bar{\psi}_{P}^{+}$in $\Delta\left(\mu_{P}^{+}\right)$has a $\Delta\left(\alpha_{P}\right)$-equivariant extension to a character $\mathrm{E}_{P}\left(\chi_{P}^{+} \times \bar{\psi}_{P}^{+}\right)$of its stabilizer $\Delta\left(\alpha_{P}\right)_{\chi_{P}^{+} \times \bar{\psi}_{P}^{+}}$in $\Delta\left(\alpha_{P}\right)$

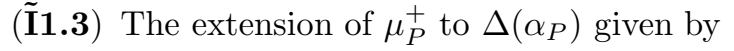

$$
\mathrm{E}_{P}\left(\mu_{P}^{+}\right)=\sum_{\chi_{P}^{+} \times \bar{\psi}_{P}^{+} \in \Delta\left(\mu_{P}^{+}\right)} \epsilon_{\mu_{P}^{+}}\left(\chi_{P}^{+}\right) \mathrm{E}_{P}\left(\chi_{P}^{+} \times \bar{\psi}_{P}^{+}\right),
$$

where $\mathrm{E}_{P}\left(\chi_{P}^{+} \times \bar{\psi}_{P}^{+}\right)(x, y)=0$ for $(x, y) \notin \Delta\left(\alpha_{P}\right)_{\chi_{P}^{+} \times \bar{\psi}_{P}^{+}}$, is perfect in the sense that

(Ĩ1.3a) $\mathrm{E}_{P}\left(\mu_{P}^{+}\right)$separates $p$-singular and $p$-regular elements of $\Delta\left(\alpha_{P}\right)$, and

$(\tilde{\mathbf{I}} 1.3 \mathbf{b}) \mathrm{E}_{P}\left(\mu_{P}^{+}\right)(x, y)$ is divisible in $\mathcal{O}$ by $\left|C_{G_{P}^{+}}(x)\right|$ and $\left|C_{H_{P}^{+}}(y)\right|$ for all $(x, y)$ in $\Delta\left(\alpha_{P}\right)$.

We verify $(\tilde{\mathbf{I}} \mathbf{1})$ when $n \neq 6$. If $e=e^{*}$, then $\mu_{P}^{+}$is stable under $\Delta\left(\alpha_{P}\right)$ by the construction in $\S 5$. If $e \neq e^{*}$, then each term $\chi_{P}^{+} \times \bar{\psi}_{P}^{+}$in $\mu_{P}^{+}$is even stable under $\Delta\left(\alpha_{P}\right)$. Let $\chi_{P}^{+}$and $\psi_{P}^{+}$be characters corresponding under $\mathrm{J}_{P}^{+}$. Then $\Delta\left(\alpha_{P}\right)_{\chi_{P}^{+} \times \bar{\psi}_{P}^{+}}$ is $G_{P}^{+} \times H_{P}^{+}$or $\Delta\left(\alpha_{P}\right)$. In the first case we set $\mathrm{E}_{P}\left(\chi_{P}^{+} \times \bar{\psi}_{P}^{+}\right)=\chi_{P}^{+} \times \bar{\psi}_{P}^{+}$. In the second case $\chi_{P}^{+}$and $\psi_{P}^{+}$extend to irreducible characters $\chi_{P}$ and $\psi_{P}$ in $\left(G_{P}, e_{P}\right)^{\vee}$ and $\left(H_{P}, f_{P}\right)^{\vee}$ corresponding under $\mu_{P}$, and we set

$$
\mathrm{E}_{P}\left(\chi_{P}^{+} \times \bar{\psi}_{P}^{+}\right)=\operatorname{Res}_{\Delta\left(\alpha_{P}\right)}^{G_{P} \times H_{P}}\left(\chi_{P} \times \bar{\psi}_{P}\right) .
$$


By (1D) and (2.3)

$$
\mathrm{E}_{P}\left(\mu_{P}^{+}\right)(x, y)=\left\{\begin{array}{cc}
\mu_{P}(x, y) & \text { if } e \neq e^{*} \\
\frac{1}{2} \mu_{P}(x, y)+\frac{1}{2} \Delta\left(\mu_{P}^{+}\right)(x, y) & \text { if } e=e^{*}
\end{array}\right.
$$

where $\Delta\left(\mu_{P}^{+}\right)$is the generalized character of $G_{P}^{+} \times H_{P}^{+}$of $(2 \mathrm{~B})$ extended by zero to $\Delta\left(\alpha_{P}\right)$. Since $\mu_{P}$ and $\Delta\left(\mu_{P}^{+}\right)$are perfect, it follows that all conditions of $(\tilde{\mathbf{I}} \mathbf{1})$ hold.

The third type $(\tilde{\mathbf{G}} \mathbf{1})$ and $(\tilde{\mathbf{G}} \mathbf{2})$ are glueing conditions on the extensions in $(\tilde{\mathbf{I}} \mathbf{1})$.

( $\tilde{\mathbf{G}} \mathbf{1}) \mathrm{E}_{\langle 1\rangle}\left(\mu_{\langle 1\rangle}^{+}\right)\left(x x^{\prime}, y y^{\prime}\right)=0$ for all $\left(x x^{\prime}, y y^{\prime}\right) \in \Delta(\alpha)$ such that $x \in G_{p}^{+}, y \in$ $H_{p}^{+}, x^{\prime} \in \tilde{G}_{x, p^{\prime}}, y^{\prime} \in \tilde{H}_{y, p^{\prime}}$, and $x \not y$ in $G^{+}$.

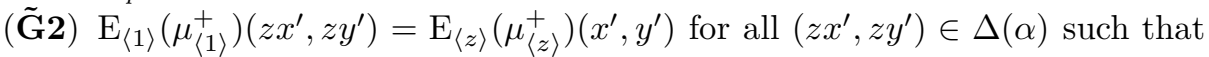
$z \in D, x^{\prime} \in C_{\tilde{X}}(z)_{p^{\prime}}, y^{\prime} \in C_{\tilde{Y}}(z)_{p^{\prime}}$.

We verify $(\tilde{\mathbf{G}} \mathbf{1})$ and $(\tilde{\mathbf{G}} \mathbf{2})$ when $n \neq 6$. Suppose $e \neq e^{*}$. Then $\mathrm{E}_{\langle 1\rangle}\left(\mu_{\langle 1\rangle}^{+}\right)=$ $\operatorname{Res}_{\Delta(\alpha)}^{G \times H}(\mu)$ and $\mathrm{E}_{P}\left(\mu_{P}^{+}\right)=\operatorname{Res}_{\Delta\left(\alpha_{P}\right)}^{G_{P} \times H_{P}}\left(\mu_{P}\right)$, so $(\tilde{\mathbf{G}} \mathbf{2})$ holds. Let $\left(x x^{\prime}, y y^{\prime}\right) \in \Delta(\alpha)$ be as in $(\tilde{\mathbf{G}} \mathbf{1})$. Since $G_{x} \not \leq G^{+}$when $\left|\Pi_{0}\right| \geq 3$, it follows that $x \not y$ in $G$, and $(\tilde{\mathbf{G}} \mathbf{1})$ holds. Suppose $e=e^{*}$. Let $\left(x x^{\prime}, y y^{\prime}\right) \in \Delta(\alpha)$ be as in $(\tilde{\mathbf{G}} \mathbf{1})$. If $x \nsim y$ in $G$, then $\mu_{\langle 1\rangle}\left(x x^{\prime}, y y^{\prime}\right)=0$. Moreover, $\Delta\left(\mu_{\langle 1\rangle}^{+}\right)\left(x x^{\prime}, y y^{\prime}\right)=0$, since each summand

$$
\left(\chi_{+}-\chi_{-}\right)\left(x x^{\prime}\right)\left(\bar{\psi}_{+}-\bar{\psi}_{-}\right)\left(y y^{\prime}\right)
$$

in $\Delta\left(\mu_{\langle 1\rangle}^{+}\right)\left(x x^{\prime}, y y^{\prime}\right)$ is zero by $(4 \mathrm{~A}),(4 \mathrm{~F}),(4 \mathrm{G})$, or $(4 \mathrm{H})$. Thus $\mathrm{E}_{\langle 1\rangle}\left(\mu_{\langle 1\rangle}^{+}\right)\left(x x^{\prime}, y y^{\prime}\right)=$ 0 by $(6.1)$, and $(\tilde{\mathbf{G}} \mathbf{1})$ holds. If $x \sim y$ in $G$, then $n=p+1$ or $p$; otherwise $G_{x}>G_{x}^{+}$ and $x \sim y$ in $G^{+}$. In this case $(G, e)^{\vee}=\left\{\chi_{1}, \chi_{2}, \ldots, \chi_{p}\right\}$, where $\chi_{i}$ is parametrized by the partition $\lambda_{i}$ of $n$ with a $p$-hook of leg length $i-1$. So $\operatorname{Res}_{G^{+}}^{G}\left(\chi_{i}\right)=\chi_{i}^{+}$is irreducible for $1 \leq i \leq p^{*}-1, \operatorname{Res}_{G^{+}}^{G}\left(\chi_{p^{*}}\right)=\chi_{p^{*},+}+\chi_{p^{*},-}$ is reducible, and

$$
\left(G^{+}, e^{+}\right)^{\vee}=\left\{\chi_{1}^{+}, \chi_{2}^{+}, \ldots, \chi_{p^{*}-1}^{+}, \chi_{p^{*},+}, \chi_{p^{*},-}\right\}
$$

$(H, f)^{\vee}$ may be identified with $Y^{\vee}=\left\{\xi_{1}, \xi_{2}, \ldots, \xi_{p}\right\}$. So $\operatorname{Res}_{H^{+}}^{H}\left(\xi_{i}\right)=\xi_{i}^{+}$is irreducible for $1 \leq i \leq p^{*}-1 \operatorname{Res}_{H^{+}}^{H}\left(\xi_{p^{*}}\right)=\xi_{p^{*},+}+\xi_{p^{*},-}$ is reducible, and

$$
\left(H^{+}, f^{+}\right)^{\vee}=\left\{\xi_{1}^{+}, \xi_{2}^{+}, \ldots, \xi_{p^{*}-1}^{+}, \xi_{p^{*},+}, \xi_{p^{*},-}\right\} .
$$

The isometries $\mu$ and $\mu^{+}$are

$$
\begin{aligned}
& \chi_{i} \mapsto(-1)^{i+1} \xi_{i} \quad \text { for } i \neq p^{*}, \quad \chi_{p^{*}} \mapsto(-1)^{p^{*}} \xi_{p^{*}} \\
& \chi_{i}^{+} \mapsto(-1)^{i+1} \xi_{i}^{+} \quad \text { for } i \neq p^{*} \quad \chi_{p^{*}, \pm} \mapsto-\epsilon_{p} \xi_{p^{*}, \pm},
\end{aligned}
$$

A direct calculation gives $(\tilde{\mathbf{G}} \mathbf{1})$.

Suppose $\left(z x^{\prime}, z y^{\prime}\right) \in \Delta(\alpha)$, where $z \in D, x^{\prime} \in G_{z, p^{\prime}}, y^{\prime} \in H_{z, p^{\prime}}$. If $x^{\prime} \notin G^{+}$and $y^{\prime} \notin H^{+}$, then

$$
\mathrm{E}_{\langle 1\rangle}\left(\mu_{\langle 1\rangle}^{+}\right)\left(z x^{\prime}, z y^{\prime}\right)=\mathrm{E}_{\langle z\rangle}\left(\mu_{\langle z\rangle}^{+}\right)\left(x^{\prime}, y^{\prime}\right)
$$


by (6.1), since $\mu_{\langle 1\rangle}\left(z x^{\prime}, z y^{\prime}\right)=\mu_{\langle z\rangle}\left(x^{\prime}, y^{\prime}\right)$. If $x^{\prime} \in G^{+}$and $y^{\prime} \in H^{+}$, then (6.2) is just $\mu_{\langle 1\rangle}^{+}\left(z x^{\prime}, z y^{\prime}\right)=\mu_{\langle z\rangle}^{+}\left(x^{\prime}, y^{\prime}\right)$. Thus $(\tilde{\mathbf{G}} \mathbf{2})$ holds.

We now suppose $n=6$. Then $G^{+} \simeq A(6) \simeq \operatorname{PSL}(2,9), \tilde{G}=\operatorname{Aut} G^{+} \simeq \operatorname{P\Gamma L}(2,9)$, and $p=3$ or 5 . Let $G_{1}, G_{2}, G_{3}$ be the maximal subgroups of $\tilde{G}$ containing $G^{+}$, labeled so that

$$
G_{1} \simeq S(6), \quad G_{2} \simeq \operatorname{PGL}(2,9), \quad G_{3} \simeq \mathrm{M}_{10}
$$

We follow Atlas notation [7, page 5] for the character table of $\tilde{G}$ :

TABle (6.3). $\tilde{G}$

\begin{tabular}{|c|c|c|c|c|c|c|c|c|c|c|c|c|c|c|c|c|c|c|c|c|c|c|c|}
\hline 360 & 8 & 9 & 9 & 4 & & 5 & 5 & & 24 & 24 & 4 & 3 & 3 & & 10 & 4 & 4 & 5 & 5 & & 2 & 4 & 4 \\
\hline $1 A$ & $2 A$ & $3 A$ & $3 B$ & $4 A$ & 5 & & $B^{*}$ & & $B$ & $2 C$ & $4 B$ & $6 A$ & $6 B$ & & $2 D$ & $8 A$ & $B^{*}$ & $10 \mathrm{~A}$ & $B^{*}$ & & $4 C$ & $8 C$ & $D^{* *}$ \\
\hline 1 & 1 & 1 & 1 & 1 & & 1 & 1 & & 1 & 1 & 1 & 1 & 1 & 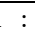 & 1 & 1 & 1 & 1 & 1 & & 1 & 1 & 1 \\
\hline 5 & 1 & 2 & -1 & -1 & ( & ) & 0 & & 3 & -1 & 1 & 0 & -1 & $\bullet$ & 0 & 0 & 0 & 0 & 0 & 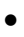 & 0 & 0 & 0 \\
\hline 5 & 1 & -1 & 2 & -1 & & ) & 0 & & -1 & 3 & 1 & -1 & 0 & $\bullet$ & 0 & 0 & 0 & 0 & 0 & $\bullet$ & 0 & 0 & 0 \\
\hline 8 & 0 & -1 & -1 & 0 & $-b$ & & $*$ & • & 0 & 0 & 0 & 0 & 0 & : & 2 & 0 & 0 & $b 5$ & $*$ & $\bullet$ & 0 & 0 & 0 \\
\hline 8 & 0 & -1 & -1 & 0 & & $k-$ & $-b 5$ & • & 0 & 0 & 0 & 0 & 0 & ) & 2 & 0 & 0 & $*$ & $b 5$ & • & 0 & 0 & 0 \\
\hline 9 & 1 & 0 & 0 & 1 & - & & -1 & & 3 & 3 & -1 & 0 & 0 & ) & -1 & 1 & 1 & -1 & -1 & & 1 & -1 & -1 \\
\hline 10 & & 1 & 1 & 0 & & ) & 0 & & 2 & -2 & 0 & -1 & 1 & : & 0 & $r 2$ & $-r 2$ & 0 & 0 & & 0 & $i 2$ & $-i 2$ \\
\hline
\end{tabular}

The columns correspond to conjugacy classes $C$ of $\tilde{G}$, the first two entries in each column being $\left|C_{G^{+}}(g)\right|$ for $g$ in $C$ and the Atlas label of the class. Also $b 5=$ $\frac{1}{2}(-1+\sqrt{5}), r 2=\sqrt{2}, i 2=i \sqrt{2}$.

The natural inclusion of $\tilde{H}=N_{\tilde{G}}(D)$ in $\tilde{G}$ induces an isomorphism $\alpha: \tilde{G} / G^{+} \rightarrow$ $\tilde{H} / H^{+}$since $\tilde{G}=\tilde{H} G^{+}$. Also $H^{+} \simeq 3^{2}: 4, \tilde{H} \simeq 3^{2}:\left[2^{4}\right]$ if $p=3$, and $H^{+} \simeq D_{10}$, $\tilde{H} \simeq 10: 4$ if $p=5$. Let $H_{i}=N_{G_{i}}(D)$, so that

$$
\begin{aligned}
& H_{1} \simeq 3^{2}: D_{8}, \quad H_{2} \simeq 3^{2}: 8, \quad H_{3} \simeq 3^{2}: Q_{8} \quad \text { if } p=3, \\
& H_{1} \simeq 5: 4, \quad H_{2} \simeq D_{20}, \quad H_{3} \simeq 5: 4 \quad \text { if } p=5 \text {. }
\end{aligned}
$$

Let $\alpha_{i}$ be the restriction of $\alpha$ to $G_{i} / G^{+}$for $i=1,2,3$, and set

$$
\Delta\left(\alpha_{i}\right)=\left\{(x, y): x \in G_{i}, y \in H_{i}, \alpha_{i}\left(x G^{+}\right)=y H^{+}\right\}
$$

We verify the compatibility conditions $(\tilde{\mathbf{I}} \mathbf{1}),(\tilde{\mathbf{G}} \mathbf{1})$, and $(\tilde{\mathbf{G}} \mathbf{2})$ for the two cases $p=3$ and $p=5$.

Suppose $p=3$. Then $D \simeq 3^{2}, H^{+} \simeq 3^{2}: 4$, and $\tilde{H} \simeq 3^{2}:\left[2^{4}\right]$. The characters 1 , $5_{1}, 5_{2}, 8_{1}, 8_{2}, 10$ in $\left(G^{+}, e^{+}\right)^{\vee}$ have stabilizers $\tilde{G}, G_{1}, G_{1}, G_{2}, G_{2}, \tilde{G}$ in $\tilde{G}$. Only the characters $1,5_{1}, 5_{2}, 8_{1}, 8_{2}$ extend to their stabilizers. Let $\mathrm{E}(1), \mathrm{E}\left(5_{1}\right), \mathrm{E}\left(5_{2}\right)$, $\mathrm{E}\left(8_{1}\right), \mathrm{E}\left(8_{2}\right)$ be the extensions given in (6.3). The following is the character table of $\tilde{H}$ : 
TABLE (6.4). $\tilde{H}, p=3$

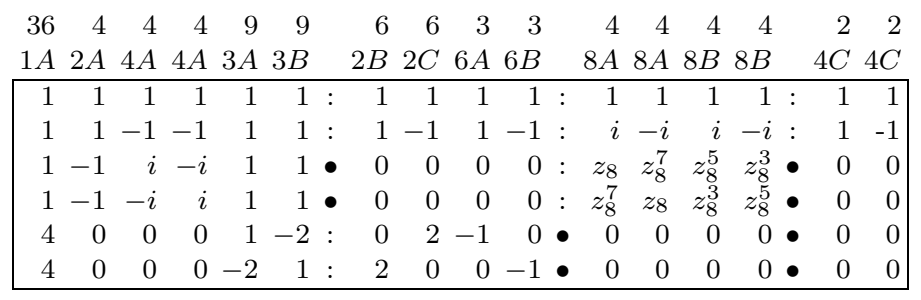

The columns correspond to conjugacy classes $C$ of $\tilde{H}$, the first two entries being $\left|C_{H^{+}}(h)\right|$ for $h$ in $C$ and the Atlas name of the $\tilde{G}$-class containing $C$. The characters $1_{1}, 1_{2}, 1_{3}, 1_{4}, 4_{1}, 4_{2}$ in $\left(H^{+}, f^{+}\right)^{\vee}$ have stabilizers $\tilde{H}, \tilde{H}, H_{2}, H_{2}, H_{1}, H_{1}$ in $\tilde{H}$. Only the characters $1_{1}, 1_{3}, 1_{4}, 4_{1}, 4_{2}$ extend to their stabilizers. Let $\mathrm{E}\left(1_{1}\right), \mathrm{E}\left(1_{3}\right)$, $\mathrm{E}\left(1_{4}\right), \mathrm{E}\left(4_{1}\right), \mathrm{E}\left(4_{2}\right)$ be the extensions given in (6.4). The isometry $\mu^{+}=\left(\mathrm{J}^{+}, \epsilon^{+}\right)$

$$
1 \mapsto 1_{1}, \quad 10 \mapsto 1_{2}, \quad 5_{1} \mapsto-4_{2}, \quad 5_{2} \mapsto-4_{1}, \quad 8_{1} \mapsto-1_{3}, \quad 8_{2} \mapsto-1_{4}
$$

is clearly stable under $\Delta(\alpha)$.

Let $\chi^{+}$and $\psi^{+}$be characters in $\left(G^{+}, e^{+}\right)^{\vee}$ and $\left(H^{+}, f^{+}\right)^{\vee}$ corresponding under $\mathrm{J}^{+}$, and set

$$
\mathrm{E}\left(\chi^{+} \times \overline{\psi^{+}}\right)=\left\{\begin{array}{l}
\mathrm{E}\left(\chi^{+}\right) \times \overline{\mathrm{E}\left(\psi^{+}\right)}, \\
\operatorname{sgn}_{\Delta\left(\alpha_{1}\right)}\left(\mathrm{E}\left(\chi^{+}\right) \times \overline{\mathrm{E}\left(\psi^{+}\right)}\right),
\end{array}\right.
$$

according as $\left(\chi^{+}, \psi^{+}\right)=\left(1,1_{1}\right),\left(8_{1}, 1_{3}\right),\left(8_{2}, 1_{4}\right)$ or $\left(\chi^{+}, \psi^{+}\right)=\left(5_{1}, 4_{2}\right),\left(5_{2}, 4_{1}\right)$. Here the characters on the right-hand side are to be restricted to the stabilizer of $\chi^{+} \times \overline{\psi^{+}}$in $\Delta(\alpha)$. The character $10 \times 1_{2}$ extends to a character $\mathrm{E}\left(10 \times 1_{2}\right)$ of $\Delta(\alpha)$. Indeed, 10 and $1_{2}$ extend to characters $\mathrm{E}_{1}(10)$ and $\mathrm{E}_{1}\left(1_{2}\right)$ of $G_{1}$ and $H_{1}$, where $\mathrm{E}_{1}(10)$ and $\mathrm{E}_{1}\left(1_{2}\right)$ are the extensions given in Tables (6.3) and (6.4). Then

$$
\mathrm{E}_{1}\left(10 \times 1_{2}\right)=\operatorname{Res}_{\Delta\left(\alpha_{1}\right)}^{G_{1} \times H_{1}}\left(\mathrm{E}_{1}(10) \times \mathrm{E}_{1}\left(1_{2}\right)\right)
$$

is $\Delta(\alpha)$-stable, so that $\mathrm{E}_{1}\left(10 \times 1_{2}\right)$ extends to a character $\mathrm{E}\left(10 \times 1_{2}\right)$ of $\Delta(\alpha)$. The assignment $\chi^{+} \times \overline{\psi^{+}} \mapsto \mathrm{E}\left(\chi^{+} \times \overline{\psi^{+}}\right)$is $\Delta(\alpha)$-equivariant, and we may define

$$
\mathrm{E}\left(\mu^{+}\right)=\sum_{\chi^{+} \in\left(G^{+}, e^{+}\right)^{\vee}} \epsilon^{+}\left(\chi^{+}\right) \mathrm{E}\left(\chi^{+} \times \overline{\psi^{+}}\right) .
$$

$\mathrm{E}\left(\mu^{+}\right)(x, y)$ satisfies conditions ( $(\tilde{\mathbf{I}} 1.3 \mathbf{a})$ and $(\tilde{\mathbf{I}} \mathbf{1} \mathbf{3 b})$ for $(x, y) \in \Delta(\alpha)-\left(G^{+} \times H^{+}\right)$. Indeed, it suffices to take $(x, y) \in \Delta\left(\alpha_{1}\right)-\left(G^{+} \times H^{+}\right)$, since $x$ in $\tilde{G}-G^{+}$commutes with a non-trivial 3-element only if $x \in G_{1}$.

Let $Q=\langle z\rangle$ be a non-trivial cyclic subgroup of $D$. Then

$$
G_{Q}^{+}=H_{Q}^{+}=Q \times Q^{\prime} \simeq C_{3} \times C_{3}, \quad \tilde{G}_{Q}=\tilde{H}_{Q}=Q \times Q^{\prime}\langle t\rangle \simeq C_{3} \times S_{3},
$$

where $t$ inverts $Q^{\prime}$. In particular, $\tilde{G}_{Q} \leq G_{1}$ and $\tilde{H}_{Q} \leq H_{1}$. Moreover, $e_{Q}^{+}=$ $f_{Q}^{+}=1, \mu_{Q}^{+}=\sum_{\zeta \in\left(Q \times Q^{\prime}\right) \vee}(\zeta \times \bar{\zeta})$ is $\Delta\left(\alpha_{Q}\right)$-stable, and $\mu_{Q}^{+}(1,1)=9$. If $\zeta^{t} \neq \zeta$, then $Q^{\prime} \not \leq \operatorname{ker} \zeta$ and $\Delta\left(\alpha_{Q}\right)_{\zeta \times \bar{\zeta}}=G_{Q}^{+} \times H_{Q}^{+}$. If $\zeta^{t}=\zeta$, then $Q^{\prime} \leq \operatorname{ker} \zeta$ and $\Delta\left(\alpha_{Q}\right)_{\zeta \times \bar{\zeta}}=\Delta\left(\alpha_{Q}\right)$. In this case $\zeta \times \bar{\zeta}$ extends to a character $\mathrm{E}_{Q}(\zeta \times \bar{\zeta})$ containing $\left(Q^{\prime} \times Q^{\prime}\right)\langle(t, t)\rangle$ in its kernel. It follows easily that conditions $(\tilde{\mathbf{I}} \mathbf{1} . \mathbf{3 a})$ and $(\tilde{\mathbf{I}} \mathbf{1} . \mathbf{3} \mathbf{b})$ hold. Moreover, $\mathrm{E}_{Q}\left(\mu_{Q}^{+}\right)(t, t)=3$, so that $(\tilde{\mathbf{G}} \mathbf{1})$ and $(\tilde{\mathbf{G}} \mathbf{2})$ also hold. 
Suppose $p=5$. Then $D \simeq 5, H^{+} \simeq D_{10}$, and $\tilde{H} \simeq 10: 4$. The characters $1,9,8_{1}$, $8_{2}$ in $\left(G^{+}, e^{+}\right)^{\vee}$ have stabilizers $\tilde{G}, \tilde{G}, G_{2}, G_{2}$ in $\tilde{G}$ and extend to their stabilizers. Let $\mathrm{E}(1), \mathrm{E}(9), \mathrm{E}\left(8_{1}\right), \mathrm{E}\left(8_{2}\right)$ be the extensions given in (6.3). The following is the character table of $\tilde{H}$ :

TABLE (6.5). $\tilde{H}, p=5$

\begin{tabular}{|c|c|c|c|c|c|c|c|c|c|c|c|c|}
\hline $\begin{array}{r}10 \\
1 A\end{array}$ & $\begin{array}{r}2 \\
2 A\end{array}$ & $\begin{array}{r}5 \\
5 A\end{array}$ & $\begin{array}{r}5 \\
B^{*}\end{array}$ & & $\begin{array}{r}2 \\
4 B\end{array}$ & $\begin{array}{r}2 \\
4 B\end{array}$ & $\begin{array}{r}10 \\
2 D\end{array}$ & $\begin{array}{r}2 \\
2 A\end{array}$ & $\begin{array}{r}5 \\
10 A\end{array}$ & $\begin{array}{r}5 \\
B^{*}\end{array}$ & $\begin{array}{r}2 \\
4 C\end{array}$ & $\begin{array}{r}2 \\
4 C\end{array}$ \\
\hline 1 & 1 & 1 & 1 & : & 1 & 1 & 1 & 1 & 1 & 1 & : & 1 \\
\hline 1 & -1 & 1 & 1 & : & $i$ & $-i$ & 1 & -1 & 1 & 1 & $i$ & $-\mathrm{i}$ \\
\hline 2 & 0 & $b 5$ & $*$ & • & 0 & 0 & -2 & 0 & $-b 5$ & $*$ & 0 & 0 \\
\hline 2 & 0 & $*$ & $b 5$ & • & 0 & 0 & -2 & 0 & $*$ & $-b 5$ & 0 & 0 \\
\hline
\end{tabular}

The characters $1_{1}, 1_{2}, 2_{1}, 2_{2}$ in $\left(H^{+}, f^{+}\right)^{\vee}$ have stabilizers $\tilde{H}, \tilde{H}, H_{2}, H_{2}$ in $\tilde{H}$ and extend to their stabilizers. Let $\mathrm{E}\left(1_{1}\right), \mathrm{E}\left(1_{2}\right), \mathrm{E}\left(2_{1}\right), \mathrm{E}\left(2_{2}\right)$ be the extensions given in (6.5). The isometry $\mu^{+}=\left(\mathrm{J}_{\mu^{+}}, \epsilon_{\mu^{+}}\right)$maps

$$
1 \mapsto 1_{1}, \quad 9 \mapsto-1_{2}, \quad 8_{1} \mapsto-2_{1}, \quad 8_{2} \mapsto-2_{2} .
$$

$\mu^{+}$is clearly $\Delta(\alpha)$-stable, and

$$
\mathrm{E}\left(\mu^{+}\right)=\mathrm{E}(1) \times \mathrm{E}\left(1_{1}\right)-\mathrm{E}(9) \times \mathrm{E}\left(1_{2}\right)-\mathrm{E}\left(8_{1}\right) \times \mathrm{E}\left(2_{1}\right)-\mathrm{E}\left(8_{2}\right) \times \mathrm{E}\left(2_{2}\right),
$$

where the right-hand side is suitably interpreted on $\Delta(\alpha)$. Since $\Delta\left(\alpha_{1}\right)-(G \times H)$ and $\Delta\left(\alpha_{3}\right)-(G \times H)$ contain no elements centralizing a 5 -element, the conditions $(\tilde{\mathbf{I}} 1.3 \mathbf{a})$ and $(\tilde{\mathbf{I}} \mathbf{1} \mathbf{3 b})$ on $\mathrm{E}\left(\mu^{+}\right)$need only be checked on $(g, h)$ in $\Delta\left(\alpha_{2}\right)$. This is straightforward.

The only non-trivial cyclic subgroup of $D$ is $D$ itself. Then

$$
G_{D}^{+}=H_{D}^{+}=D, \quad \tilde{G}_{D}=\tilde{H}_{D}=D \times\langle t\rangle \simeq C_{10} .
$$

In particular, $\tilde{G}_{D} \leq G_{2}$ and $\tilde{H}_{D} \leq H_{2}$. Moreover, $e_{D}^{+}=f_{D}^{+}=1, \mu_{D}^{+}$is the identity isometry, $\mu_{D}^{+}$is $\Delta\left(\alpha_{D}\right)$-stable, and $\mu_{D}^{+}(1,1)=5$. We may choose $\mathrm{E}_{Q}\left(\mu_{Q}^{+}\right)$ as $\sum_{\zeta}(\zeta \times \bar{\zeta})$, where $\zeta$ runs over the characters of $D \times\langle t\rangle$ with $\langle t\rangle$ in their kernels. Then $\mathrm{E}_{D}\left(\mu_{D}^{+}\right)(t, t)=5$. The conditions $(\tilde{\mathbf{I}} \mathbf{1}),(\tilde{\mathbf{G}} \mathbf{1}),(\tilde{\mathbf{G}} \mathbf{2})$ now follow.

\section{The isotypy conjeCture for alternating groups for $p=2$}

Let $G=S(\Pi)=S_{n}$ and $G^{+}=A(\Pi)=A_{n}$ be the symmetric group and alternating group on the set $\Pi$ of size $n$, and let $p=2$. The 2-blocks of $\mathcal{O} G$ are self-dual since 2-cores are self-dual. Thus each block of $\mathcal{O} G^{+}$is covered by a unique block of $\mathcal{O} G$. Suppose $e$ is a block of $\mathcal{O} G$ parametrized by the 2-core $\lambda_{0}$. If $e$ has defect 0 , then $\operatorname{Res}_{G^{+}}^{G}\left(\chi_{\lambda_{0}}\right)=\chi_{\lambda_{0},+}+\chi_{\lambda_{0},-}$ and $e$ covers two blocks of $\mathcal{O} G^{+}$ of defect 0 , namely the blocks $e_{ \pm}$containing $\chi_{\lambda_{0}, \pm}$. If $e$ has positive defect, then $(G, e)^{\vee}$ contains characters $\chi_{\lambda}$ which do not split on $G^{+}$, and $e$ covers a unique block $e^{+}$of $\mathcal{O} G^{+}$. Thus a block of $\mathcal{O} G$ of positive defect covers a unique $G$-stable block of $\mathcal{O} G^{+}$. Conversely a $G$-stable block of $\mathcal{O} G^{+}$is covered by a unique block of $\mathcal{O} G$ of positive defect.

We consider a block of $\mathcal{O} G^{+}$with abelian defect group. If the block is not $G$ stable, then it has defect 0 and is $e_{ \pm}$for some block $e$ of $\mathcal{O} G$ of defect 0 . If the block is $G$-stable, then it is $e^{+}$for a unique block $e$ of $\mathcal{O} G$ of positive defect. Let 
$w$ be the weight of $e$ and let $\sum_{i \geq 1} 2^{i} w_{i}$ be the 2-adic expansion of $2 w$. Then $e$ has defect group

$$
D=\prod_{i \geq 1}\left(T_{i}\right)^{w_{i}}
$$

where $T_{i}=Z_{2} \nmid Z_{2} \nmid \cdots \prec Z_{2}(i$ times $)$ is a Sylow 2-subgroup of $S_{2^{i}}$, and $e^{+}$has defect group $D^{+}=D \cap G^{+}<D$.

Lemma (7A). Suppose $D^{+}$is abelian. Then either $D^{+}=1$ and $D=T_{1}$, or $D^{+} \simeq Z_{2} \times Z_{2}$ and $D=T_{2}$.

Proof. $T_{i}$ is non-abelian for $i \geq 2$ and $\left[T_{i}, T_{i}\right]$ is non-abelian for $i \geq 3$. Thus $D$ is $T_{1}, T_{2}$, or $T_{1} \times T_{2}$. The case $D=T_{1} \times T_{2}$ is impossible since $D^{+}$would have a non-abelian section isomorphic to $T_{2}$. Thus $D=T_{1}$ or $T_{2}$.

Let $e^{+}$be a block of $\mathcal{O} G^{+}$with abelian defect group $D^{+}>1$, let $e$ be the unique block of $\mathcal{O} G$ covering $e^{+}$, and let $D$ be a defect group of $e$ such that $D^{+}=$ $D \cap G^{+}$. So (7A) implies that $D=T_{2}$ and $D^{+} \simeq Z_{2} \times Z_{2}$. Let $H=N_{G}\left(D^{+}\right)$ and $H^{+}=N_{G^{+}}\left(D^{+}\right)$. Let $\lambda_{0}$ be the 2-core parametrizing $e$. If $\left|\lambda_{0}\right|=0$, then $n=4, G=H=S_{4}, G^{+}=H^{+}=A_{4}$, and the isotypy conjecture holds trivially. If $\left|\lambda_{0}\right|=1$, then $n=5, G=S_{5}, G^{+}=A_{5}, H=S_{4}, H^{+}=A_{4}$, and the isotypy conjecture holds by [4, Appendice A1.1]. So we suppose $\left|\lambda_{0}\right| \geq 3$ and $n \geq 7$. Let $\Pi=\Pi_{0} \cup \Pi_{1}$, where $\Pi_{0}$ and $\Pi_{1}$ are the fixed-point set and the support of $D$. Let $H_{0}=S\left(\Pi_{0}\right)$ and $H_{1}=S\left(\Pi_{1}\right)=S_{4}$. Then $H=H_{0} \times H_{1}, H^{+}=\left(H_{0} \times H_{1}\right)^{+}$, and

$$
\begin{aligned}
C_{G}\left(D^{+}\right) & =C_{H}\left(D^{+}\right)=H_{0} \times D^{+}, \\
C_{G^{+}}\left(D^{+}\right) & =C_{H^{+}}\left(D^{+}\right)=H_{0}^{+} \times D^{+} .
\end{aligned}
$$

Let $\chi_{\lambda_{0}}$ be the character of $H_{0}$ parametrized by $\lambda_{0}$, let $1_{H_{1}}$ be the 1 -character of $H_{1}$, and let $f$ be the block of $\mathcal{O H}$ containing $\chi_{\lambda_{0}} \times 1_{H_{1}}$. Then $f$ covers a unique block $f^{+}$of $\mathcal{O} H^{+}$since $\operatorname{Res}_{H^{+}}^{H}\left(\chi_{\lambda_{0}} \times 1_{H_{1}}\right)$ is irreducible, and $f$ and $f^{+}$are the Brauer correspondents of $e$ and $e^{+}$. We take as maximal $e^{+}$-subpair $\left(D^{+}, e_{D^{+}}^{+}\right)$and maximal $f^{+}$-subpair $\left(D^{+}, f_{D^{+}}^{+}\right)$the subpair $\left(D^{+}, e_{\lambda_{0},+}\right)$, where $e_{\lambda_{0},+}$ is the block of $\mathcal{O}\left(H_{0}^{+} \times D^{+}\right)$containing $\chi_{\lambda_{0},+} \times 1_{D^{+}}$.

The partitions $\lambda$ with 2-core $\lambda_{0}$ have the following 2-quotients:

$$
(2, \emptyset), \quad\left(1^{2}, \emptyset\right), \quad(1,1), \quad(\emptyset, 2), \quad\left(\emptyset, 1^{2}\right) .
$$

We label the $\chi_{\lambda}$ by the 2-quotients of $\lambda$ as $\chi_{(2, \emptyset)}, \chi_{\left(1^{2}, \emptyset\right)}, \chi_{(1,1)}, \chi_{(\emptyset, 2)}, \chi_{\left(\emptyset, 1^{2}\right)}$. In particular, $\chi_{(2, \emptyset)}^{*}=\chi_{\left(\emptyset, 1^{2}\right)}, \chi_{\left(1^{2}, \emptyset\right)}^{*}=\chi_{(\emptyset, 2)}, \chi_{(1,1)}^{*}=\chi_{(1,1)}$. So $\left(G^{+}, e^{+}\right)^{\vee}$ consists of the four characters

$$
\chi_{(2, \emptyset)}^{+}=\operatorname{Res}_{G^{+}}^{G}\left(\chi_{(2, \emptyset)}\right), \quad \chi_{\left(1^{2}, \emptyset\right)}^{+}=\operatorname{Res}_{G^{+}}^{G}\left(\chi_{\left(1^{2}, \emptyset\right)}\right), \quad \chi_{(1,1), \pm},
$$

where $\chi_{(1,1), \pm}$ are the constituents of $\operatorname{Res}_{G^{+}}^{G}\left(\chi_{(1,1)}\right)$.

The irreducible characters in $(H, f)^{\vee}$ are

$$
\chi_{\lambda_{0}} \times \chi_{(4)}, \quad \chi_{\lambda_{0}} \times \chi_{(3,1)}, \quad \chi_{\lambda_{0}} \times \chi_{\left(2^{2}\right)}, \quad \chi_{\lambda_{0}} \times \chi_{\left(2,1^{2}\right)}, \quad \chi_{\lambda_{0}} \times \chi_{\left(1^{4}\right)},
$$

where the second factors are the irreducible characters of $S\left(\Pi_{1}\right)$. Since the partitions (4), $(3,1),\left(2^{2}\right),\left(2,1^{2}\right),\left(1^{4}\right)$ have 2 -quotients $(2, \emptyset),\left(1^{2}, \emptyset\right),(1,1),(\emptyset, 2),\left(\emptyset, 1^{2}\right)$, we also label these characters in $(H, f)^{\vee}$ as $\psi_{(2, \emptyset)}, \psi_{\left(1^{2}, \emptyset\right)}, \psi_{(1,1)}, \psi_{(\emptyset, 2)}, \psi_{\left(\emptyset, 1^{2}\right)}$. In 
particular, $\psi_{(2, \emptyset)}^{*}=\psi_{\left(\emptyset, 1^{2}\right)}, \psi_{\left(1^{2}, \emptyset\right)}^{*}=\psi_{(\emptyset, 2)}, \psi_{(1,1)}^{*}=\psi_{(1,1)}$. So $\left(H^{+}, f^{+}\right)^{\vee}$ consists of the four characters

$$
\psi_{(2, \emptyset)}^{+}=\operatorname{Res}_{H^{+}}^{H}\left(\psi_{(2, \emptyset)}\right), \quad \psi_{\left(1^{2}, \emptyset\right)}^{+}=\operatorname{Res}_{H^{+}}^{H}\left(\psi_{\left(1^{2}, \emptyset\right)}\right), \quad \psi_{(1,1), \pm},
$$

where $\psi_{(1,1), \pm}$ are the constituents of $\operatorname{Res}_{H^{+}}^{H}\left(\psi_{(1,1)}\right)$.

Let $\mu^{+}=\left(\mathrm{J}^{+}, \epsilon^{+}\right)$be the isometry in $\mathcal{R}_{K}\left(\left(G^{+}, e^{+}\right),\left(H^{+}, f^{+}\right)\right)$given by

$$
\chi_{(2, \emptyset)}^{+} \mapsto \psi_{(2, \emptyset)}^{+}, \quad \chi_{\left(1^{2}, \emptyset\right)}^{+} \mapsto-\psi_{\left(1^{2}, \emptyset\right)}^{+}, \quad \chi_{(1,1), \pm} \mapsto-\psi_{(1,1), \pm} .
$$

Each non-trivial cyclic subgroup $P=\langle x\rangle$ of $D^{+}$is $Z(D)^{t}$ for some $t$ in $A\left(\Pi_{1}\right)$. Thus

$$
G_{x}=H_{x}=S\left(\Pi_{0}\right) \times D^{t}, \quad G_{x}^{+}=H_{x}^{+}=\left(S\left(\Pi_{0}\right) \times D^{t}\right)^{+}
$$

and the subpairs $\left(\langle x\rangle, e_{x}^{+}\right)$and $\left(\langle x\rangle, f_{x}^{+}\right)$coincide. We take $\mu_{x}^{+}=\left(\mathrm{J}_{x}^{+}, \epsilon_{x}^{+}\right)$as the identity isometry. The diagram

$$
\begin{array}{ccc}
\mathrm{CF}\left(G^{+}, e^{+}, K\right) & \stackrel{\mathrm{R}_{\mu_{\langle 1\rangle}^{+}}}{\longrightarrow} & \mathrm{CF}\left(H^{+}, f^{+}, K\right) \\
\downarrow d_{G}^{\left(x, e_{x}^{+}\right)} & \downarrow d_{H^{+}}^{\left(x, f_{x}^{+}\right)} \\
\mathrm{CF}_{p^{\prime}}\left(G_{P}^{+}, e_{x}^{+}, K\right) \stackrel{\mathrm{R}_{\mu_{x}^{+}, p^{\prime}}}{\longrightarrow} & \mathrm{CF}_{p^{\prime}}\left(H_{P}^{+}, f_{x}^{+}, K\right)
\end{array}
$$

is then commutative. Indeed, it suffices to show that

$$
\chi^{+}(x y)=\mathrm{R}_{\mu_{\langle 1\rangle}^{+}}\left(\chi^{+}\right)(x y)
$$

for all $\chi^{+}$in $\left(G^{+}, e^{+}\right)^{\vee}$ and all $y$ in $H_{x, 2^{\prime}}^{+}$. Such $y$ 's lie in $A\left(\Pi_{0}\right)$. Now

$$
\begin{gathered}
\chi_{(2, \emptyset)}^{+}(x y)=\chi_{(2, \emptyset)}(x y)=\chi_{\lambda_{0}}(y), \\
\chi_{\left(1^{2}, \emptyset\right)}^{+}(x y)=\chi_{\left(1^{2}, \emptyset\right)}(x y)=\chi_{\lambda_{0}}(y),
\end{gathered}
$$

by the Murnaghan-Nakayama formula. On the other hand,

$$
\begin{gathered}
\psi_{(2, \emptyset)}^{+}(x y)=\psi_{(2, \emptyset)}(x y)=\chi_{\lambda_{0}}(y), \\
\psi_{\left(1^{2}, \emptyset\right)}^{+}(x y)=\psi_{\left(1^{2}, \emptyset\right)}(x y)=-\chi_{\lambda_{0}}(y),
\end{gathered}
$$

since $\chi_{(4)}(x)=1$ and $\chi_{(3,1)}(x)=-1$. Lastly,

$$
\chi_{(1,1), \pm}(x y)=\frac{1}{2} \chi_{(1,1)}(x y)=-\chi_{\lambda_{0}}(y) .
$$

The first equality holds since $x y$ has cycle type unequal to $\eta(\lambda)$, where $\lambda$ is the partition with 2-core $\lambda_{0}$ and 2-quotient $(1,1)$. The second equality holds by the Murnaghan-Nakayama formula. We may suppose with suitable labeling that

$$
\begin{aligned}
& \psi_{(1,1),+}(x y)=\chi_{\lambda_{0},+}(y) \chi_{\left(2^{2}\right),+}(x)+\chi_{\lambda_{0},-}(y) \chi_{\left(2^{2}\right),-}(x), \\
& \psi_{(1,1),-}(x y)=\chi_{\lambda_{0},+}(y) \chi_{\left(2^{2}\right),-}(x)+\chi_{\lambda_{0},-}(y) \chi_{\left(2^{2}\right),+}(x) .
\end{aligned}
$$

Both equal $\chi_{\lambda_{0}}(y)$, since $\chi_{\left(2^{2}\right), \pm}(x)=1$. Thus (7.1) is commutative. It now follows as in $\S 5$ that $e^{+}$and $f^{+}$are isotypic blocks. 


\section{REFERENCES}

1. J. L. Alperin, M. Broué, Local methods in block theory, Ann. of Math. 110 (1979), 143-157. MR 80f: 20010

2. M. Broué, Radical, hauteurs, p-sections et blocs, Ann. of Math. 107 (1978), 89-107. MR 80a:20014

3. M. Broué, Les $\ell$-blocs des groupes $G L(n, q)$ et $U\left(n, q^{2}\right)$ et leurs structures locales, Astérisque 133-134 (1986), 159-188. MR 87e:20021

4. M. Broué, Isométries parfaites, types de blocs, catégories dériveés, Astérisque 181-182 (1990), 61-91. MR 91i:20006

5. M. Broué, L. Puig, Characters and local structure in G-algebras, J. Algebra 63 (1980), 306317. MR 81j:20021

6. O. Celik, A. Kerber, H. Pahlings, Zur Darstellungstheorie gewisser Verallgemeinerungen der Serien von Weyl-Gruppen., Mitt. Math. Sem. Giessen 119 (1975), 15-90. MR 54:7600

7. J. H. Conway, R. T. Curtis, S. P. Norton, R. A. Parker, R. A. Wilson, An ATLAS of finite groups., Oxford University Press, Oxford, 1985. MR 88g:20025

8. M. Enguehard, Isométries parfaites dans les groupes symétriques, Rapport de recherche du LMENS, DMI, Ecole Normale Supérieure 88-10 (1988), 1-19. cf. MR 91d:20015

9. M. Enguehard, Isométries parfaites entres blocs de groupes lineaires ou unitaires, Math. Zeit. 204 (1991), 1-24. MR 92c:20017

10. P. Fong, M. E. Harris, On perfect isometries and isotypies in finite groups, Invent. Math. 114 (1993), 139-191. MR 94i:20012

11. G. Frobenius, Uber die Charaktere der alternirenden Gruppe, Sitzber. Preuß. Akad. Wiss. (1901), 303-315.

12. G. James, A. Kerber, The representation theory of the symmetric group, Addison-Wesley, Reading, MA, 1981. MR 83k:20003

13. L. Puig, Local block theory in p-solvable groups, Proc. Sympos. Pure Math., vol. 37, Amer. Math. Soc., Providence, RI, 1979, pp. 385-388. MR 82c:20045

14. R. Rouquier, Sur les blocs à groupe de défaut abélien dans les groupes symétriques et sporadiques, J. Algebra 168 (1994), 648-694. MR 95h:20015

15. J. Stembridge, On the eigenvalues of representations of reflection groups and wreath products, Pacific. J. of Math. 140 (1989), 353-396. MR 91a:20022

Department of Mathematics, Statistics, and Computer Science, University of Illinois at Chicago, Chicago, Illinois 60680

Department of Mathematics, University of Minnesota, Minneapolis, Minnesota 55455 